\title{
AGN jet feedback on a moving mesh: cocoon inflation, gas flows and turbulence
}

\author{
Martin A. Bourne* and Debora Sijacki \\ Institute of Astronomy and Kavli Institute for Cosmology, University of Cambridge, Madingley Road, Cambridge, CB3 OHA, UK \\ * E-mail: mabourne@ast.cam.ac.uk
}

Received

\begin{abstract}
In many observed galaxy clusters, jets launched by the accretion process on to supermassive black holes, inflate large-scale cavities filled with energetic, relativistic plasma. This process is thought to be responsible for regulating cooling losses, thus moderating the inflow of gas on to the central galaxy, quenching further star formation and maintaining the galaxy in a red and dead state. In this paper, we implement a new jet feedback scheme into the moving mesh-code AREPO, contrast different jet injection techniques and demonstrate the validity of our implementation by comparing against simple analytical models. We find that jets can significantly affect the intracluster medium (ICM), offset the overcooling through a number of heating mechanisms, as well as drive turbulence, albeit within the jet lobes only. Jet-driven turbulence is, however, a largely ineffective heating source and is unlikely to dominate the ICM heating budget even if the jet lobes efficiently fill the cooling region, as it contains at most only a few percent of the total injected energy. We instead show that the ICM gas motions, generated by orbiting substructures, while inefficient at heating the ICM, drive large-scale turbulence and when combined with jet feedback, result in line-ofsight velocities and velocity dispersions consistent with the Hitomi observations of the Perseus cluster.
\end{abstract}

Key words: black hole physics - methods: numerical - galaxies: active - galaxies: clusters: general - galaxies: clusters: intracluster medium - galaxies: jets.

\section{INTRODUCTION}

Galaxy clusters show X-ray luminosities of up to $\sim 10^{45} \mathrm{erg}$ $\mathrm{s}^{-1}$ in their centres (McNamara \& Nulsen 2007), indicating significant energy losses. Based on early X-ray observations of galaxy clusters, it was estimated that the cooling time is shorter than the Hubble time. At face value, therefore a cooling flow should be established with inflow rates of $\sim 1000$ $\mathrm{M}_{\odot} \mathrm{yr}^{-1}$ (Fabian 1994). Such cooling flows should result in significant amounts of cold molecular gas and star formation within the central cluster galaxies; however, this is not observed. Some mechanism (or combination of mechanisms) is acting to prevent gas cooling and hence maintain star formation rates at relatively modest values of $\sim 1-10 \mathrm{M}_{\odot} \mathrm{yr}^{-1}$ (e.g. McNamara \& Nulsen 2007; Donahue et al. 2010; Cooke et al. 2016; Fogarty et al. 2017), although in some brightest cluster galaxies star formation can be $\gtrsim 100 \mathrm{M}_{\odot} \mathrm{yr}^{-1}$ (e.g. Crawford et al. 1999; Egami et al. 2006; McNamara et al. 2006; von der Linden et al. 2007; Mittal et al. 2015; Fogarty et al. 2017; Mittal et al. 2017), with moderate molecular gas reservoirs within cluster cores (e.g. O'Dea et al. 2008; McNamara et al. 2014; Russell et al. 2014, 2017). Furthermore, X-ray emission lines below $1 \mathrm{keV}$, expected for low temperature gas, were not seen in $A S C A$ (e.g. Ikebe et al. 1997; Makishima et al. 2001), XMM-Newton (e.g. Peterson et al. 2001, 2003; Tamura et al. 2001; Böhringer et al. 2002; Matsushita et al. 2002) or Chandra (e.g. Lewis et al. 2002) observations, which along with UV observations (e.g. Oegerle et al. 2001; Bregman et al. 2006) suggest lower than expected cooling rates.

A number of mechanisms have been invoked to pump energy into the intracluster medium (ICM) and explain the apparent lack of cooling, including thermal conduction from hot gas at larger radii (e.g. Ruszkowski \& Begelman 2002; Zakamska \& Narayan 2003; Voigt \& Fabian 2004; Conroy \& Ostriker 2008; Bogdanović et al. 2009; Ruszkowski \& Oh 2010, 2011) and stirring by the motions of substructures (Fujita et al. 2004; Ruszkowski \& Oh 2011). However, the principle energy source is expected to be feedback from an accreting supermassive black hole (SMBH). In general, active galactic nucleus (AGN) feedback can be split into two primary modes, both of which are thought to play an important role in galaxy evolution (see e.g. Fabian 2012, for a review). The quasar mode, taking the form of powerful, isotropic winds and outflows during phases of rapid $\mathrm{BH}$ 
growth, is believed to drive observed $\mathrm{BH}$ scaling relations, such as the $M_{\mathrm{BH}}-\sigma$ and $M_{\mathrm{BH}}-M_{\mathrm{b}}$ relations (e.g. Ferrarese \& Merritt 2000; Häring \& Rix 2004; Gültekin et al. 2009; Kormendy \& Ho 2013; McConnell \& Ma 2013), and quench star formation. Whereas the maintenance mode, associated with the production of jets by moderately accreting BHs, is thought to keep the gas surrounding galaxies warm and hence prevent it from cooling on to the galaxy. AGN feedback provides an explanation for the observed discrepancy between the dark matter halo mass function and the galaxy stellar mass function (e.g. Bower et al. 2006; Croton et al. 2006) and is invoked in analytical models and simulations to inhibit cooling in galaxy clusters and ensure that early type elliptical galaxies remain red and dead (e.g. Bower et al. 2006; Croton et al. 2006; Sijacki \& Springel 2006a; Sijacki et al. 2007).

X-ray observations of galaxy clusters often show giant cavities of relativistic plasma (Fabian et al. 2000, 2011; McNamara et al. 2000; Heinz et al. 2002a; Forman et al. 2007) that are expected to be inflated by jets produced by accretion on to a central SMBH (e.g. Binney \& Tabor 1995; Omma et al. 2004; McNamara et al. 2005; Fabian et al. 2006; Sijacki \& Springel 2006a; Cattaneo \& Teyssier 2007; Forman et al. 2007; Sijacki et al. 2007; Dubois et al. 2010, 2012). It is these jets and the cocoons they inflate that are assumed to be the source of heating in galaxy clusters (e.g. Churazov et al. 2001, 2002; Bîrzan et al. 2004). The high fraction of cool core clusters that contain cavities and exhibit radio emission (see e.g. Burns 1990; Dunn et al. 2005; Dunn \& Fabian 2006, 2008; McNamara \& Nulsen 2007; Sun 2009; Fabian 2012) suggest they are a common phenomenon. The energy content of these cavities $\left(10^{55}-10^{61} \mathrm{erg}\right)$, based upon PV calculations, show a correlation with the X-ray luminosity/cooling time within the ICM (Rafferty et al. 2006; McNamara \& Nulsen 2007; Nulsen et al. 2007; Dunn \& Fabian 2008; Fabian 2012; Hlavacek-Larrondo et al. 2012) and are thus expected to be the mechanism through which cooling is regulated. Combining this with the fact that many clusters have short central cooling times suggests that self-regulation is at play and that feedback is the dominant mechanism regulating cooling and heating (see e.g. McNamara \& Nulsen 2007, for a full discussion). On top of this, many cool core clusters exhibit positive central temperature gradients (e.g. Cavagnolo et al. 2008; Hudson et al. 2010; McDonald et al. 2014), further suggesting that any central heating mechanism cannot exceed the rate of cooling.

ROSAT observations of the Perseus cluster provided the first clear evidence for X-ray cavities (Boehringer et al. 1993). Over the following decades, XMM-Newton and Chan$d r a$ delivered an ever growing and more up to date collection of galaxy cluster observations (e.g. Fabian et al. 2000, 2005a, 2006; McNamara et al. 2000; Tamura et al. 2001; Heinz et al. 2002b; Forman et al. 2007; Blanton et al. 2011; Sanders et al. 2016). Most recently, albeit short-lived, the ill-fated Hitomi mission has provided the most detailed kinematic observations of the Perseus cluster to date (Hitomi Collaboration et al. 2016), showing a relatively sedate ICM, with respect to gas motions. Combined, these observations have produced a wealth of information to aid our understanding of how X-ray cavities are inflated and interact with the ICM.

However, while such observations have provided valuable insight into the processes at play, they contain limited temporal information on the jet feedback process. Further, given observational difficulties in observing faint cavities, such as those at large distances from the cluster centre and in systems at high redshift, they provide an inherently biased view of such systems. With this in mind, it is vital to couple the latest observational results with state-of-theart computer simulations. Much numerical work has already been performed in an attempt to understand various aspects of AGN jet feedback. The large dynamic range involved in such simulations has, however, meant that simplifications often have to be made. Some simulations attempt to mimic the inflation of jet cavities by injecting off centre, hot bubbles (e.g. Quilis et al. 2001; Sijacki \& Springel 2006a; Sijacki et al. 2007, 2015), finding that this can effectively disrupt cooling flows and reduce star formation rates, even in fully self-consistent cosmological simulations. Other work, however, has specifically included the inflation and evolution of the jet cavities themselves and their subsequent impact on cluster haloes (e.g. Churazov et al. 2001; Reynolds et al. 2001; Basson \& Alexander 2003; Omma et al. 2004; Gaibler et al. 2009; Dubois et al. 2011; Hardcastle \& Krause 2013, 2014; English et al. 2016; Weinberger et al. 2017), focusing mostly on isolated halo models. Further, simulations including self-consistent feedback, in which the jet properties are linked to estimated SMBH accretion rates (e.g. Cattaneo \& Teyssier 2007; Dubois et al. 2010, 2012; Gaspari et al. 2011, 2012; Li \& Bryan 2014; Li et al. 2015; Prasad et al. 2015; Yang \& Reynolds 2016a,b), find that self-regulated SMBH growth and feedback is able to inhibit cooling and produce a number of the observed properties of galaxy clusters.

Despite the successes of such models, there is still little consensus on which processes dictate the transfer of the mechanical jet energy isotropically to the ICM (e.g. Vernaleo $\&$ Reynolds 2006) and hence dominate the heating energy budget. A number of processes have been suggested including heating due to dissipation of turbulence (e.g. Banerjee \& Sharma 2014; Zhuravleva et al. 2014), sound waves (e.g. Fabian et al. 2003, 2005b, 2017; Ruszkowski et al. 2004), or gravity waves (e.g. Omma et al. 2004), shock heating (e.g. Fabian et al. 2003; Randall et al. 2015; Li et al. 2016), mixing (e.g. Hillel \& Soker 2016, 2017a), cavity heating (e.g. Churazov et al. 2002; Bîrzan et al. 2004), cosmic ray production (e.g. Sijacki et al. 2008; Pfrommer 2013), and gas circulation (e.g. Yang \& Reynolds 2016b). However, as critically pointed out by Yang \& Reynolds (2016b), it is likely that a number of different processes are at play.

In this paper, we present a novel method of including AGN jet feedback within the moving mesh-code AREPO (Springel 2010). In Section 2, we discuss the AREPO code and outline our new jet inflation prescription, while in Section 3 , we present our first results, highlighting the robustness of our scheme by carrying out a numerical resolution study and comparing different jet injection techniques. In Section 4 , we consider jet precession and discuss the properties of inflated jet cavities, resultant gas flows and how halo properties are impacted. In Section 5, we include substructures in our galaxy cluster in order to investigate the turbulence they drive and how this impacts on jet evolution and properties. Specifically, in this section, we compare recent Hitomi observations of the velocity field in the Perseus cluster with results from our simulations. Finally, in Section 6 and Section 7 , we discuss and summarize the results of our sim- 
ulations, highlighting implications for our understanding of the role of jets in galaxy cluster evolution.

\section{NUMERICAL METHOD}

\subsection{Code}

We use the moving mesh-code AREPO, presented in Springel (2010), which employs a finite-volume solver for hydrodynamics and the TREEPM method for gravity (Springel 2005). The simulations presented in this paper rely on a key ability of the hydrodynamic solver in AREPO, namely the ability to refine and de-refine cells based on certain criteria and thus allowing adequately high resolution where needed, but computationally less expensive low resolution where possible. For most of the simulations presented in this paper, we employ primordial cooling and the subgrid interstellar medium (ISM)/star-formation model of Springel \& Hernquist (2003), which implements an effective equation of state to model the unresolved, supernova regulated, multiphase ISM and the explicit (stochastic) formation of stars as $N$-body particles (in actuality, each $N$-body particle represents a stellar population). We note that, as in Curtis \& Sijacki (2015), gas within the central refinement region (see Section 2.2.4) is not allowed to form stars in order to avoid spurious $N$-body heating effects. On top of this, we implement (and modify) a number of other techniques important to the $\mathrm{BH}$ growth and feedback, which we discuss in the following sections.

\subsection{Blackhole accretion and feedback}

All simulations presented below contain a central BH, modelled as a sink particle, which can accrete surrounding gas, acts as a source of feedback and is the BH refinement focal point (see Section 2.2.4).

\subsubsection{Accretion}

The net growth rate of a $\mathrm{BH}$ can be calculated as the difference between the gas accretion rate and mass outflow rate close to the $\mathrm{BH}$ such that

$\dot{M}_{\mathrm{BH}}=\left(1-\epsilon_{\mathrm{r}}\right) \dot{M}_{\mathrm{a}}-\dot{M}_{\mathrm{J}}$

where $\epsilon_{\mathrm{r}}$ is the radiative efficiency of accretion, $\dot{M}_{\mathrm{a}}$ is the gas accretion rate and $\dot{M}_{\mathrm{J}}$ is the rate of mass outflow in the form of a jet. Note that galaxy formation and cosmological simulations typical neglect this final term; however, as highlighted by Ostriker et al. (2010), it can be important to explicitly consider the mass outflow rate when considering AGN feedback. Similarly to Ostriker et al. (2010), we define a jet mass loading factor

$\eta_{\mathrm{J}}=\frac{\dot{M}_{\mathrm{J}}}{\dot{M}_{\mathrm{BH}}}$

Combining equations (1) and (2) results in a $\mathrm{BH}$ growth rate of

$\dot{M}_{\mathrm{BH}}=\frac{1-\epsilon_{\mathrm{r}}}{1+\eta_{\mathrm{J}}} \dot{M}_{a}$.

In what follows, BHs grow at the rate defined by equation (3) where we set $\eta_{\mathrm{J}}=1, \epsilon_{\mathrm{r}}=0.1$ and assume that $\dot{M}_{\mathrm{a}}$ is a fixed fraction of the Eddington accretion rate

$\dot{M}_{\mathrm{Edd}}=\frac{4 \pi G M_{\mathrm{BH}} m_{\mathrm{p}}}{\epsilon_{\mathrm{r}} \sigma_{\mathrm{T}} c}$,

where $G$ is the gravitational constant, $m_{\mathrm{p}}$ is the proton rest mass, $\sigma_{\mathrm{T}}$ is the Thompson scattering cross-section and $c$ is the speed of light. As such, in this work, we fix $\dot{M}_{a}=$ $0.02 \dot{M}_{\mathrm{Edd}}$, and so from equation (3) $\dot{M}_{\mathrm{BH}}=0.009 \dot{M}_{\mathrm{Edd}}$, corresponding to a jet power of $\sim 10^{45} \mathrm{erg} \mathrm{s}^{-1}$ for $M_{\mathrm{BH}}=10^{9}$ $\mathrm{M}_{\odot}$. While we do not consider self-consistent $\mathrm{BH}$ growth and feedback here, we will investigate self-regulation in future work.

\subsubsection{Physical properties of the jet energy and momentum content}

Physically, accretion on to the $\mathrm{BH}$ is expected to release energy at a rate of

$\dot{E}_{\mathrm{J}}=\epsilon_{\mathrm{f}} \epsilon_{\mathrm{r}} \dot{M}_{\mathrm{BH}} c^{2}\left(=\frac{1}{2} \dot{M}_{\mathrm{J}} v_{\mathrm{J}}^{2}\right)$,

where $\epsilon_{\mathrm{f}}=1$ is the efficiency of coupling energy to the jet and $\dot{M}_{\mathrm{J}} v_{\mathrm{J}}^{2} / 2$ is the jet kinetic energy. Combining equations (2) and (3), the mass outflow rate in the jet can be written as

$\dot{M}_{\mathrm{J}}=\eta_{\mathrm{J}} \frac{1-\epsilon_{\mathrm{r}}}{1+\eta_{\mathrm{J}}} \dot{M}_{a}$,

while the kinetic energy and momentum of the jet are given as

$\dot{E}_{\mathrm{J}}=\frac{1-\epsilon_{\mathrm{r}}}{1+\eta_{\mathrm{J}}} \epsilon_{\mathrm{f}} \epsilon_{\mathrm{r}} \dot{M}_{\mathrm{a}} c^{2}$,

and

$\dot{p}_{\mathrm{J}}=\eta_{\mathrm{J}} \frac{1-\epsilon_{\mathrm{r}}}{1+\eta_{\mathrm{J}}}\left(\frac{2 \epsilon_{\mathrm{f}} \epsilon_{\mathrm{r}}}{\eta_{\mathrm{J}}}\right)^{1 / 2} \dot{M}_{\mathrm{a}} c$

respectively, where the velocity of the sub-resolution jet would be

$v_{\mathrm{J}}=\left(\frac{2 \epsilon_{\mathrm{f}} \epsilon_{\mathrm{r}}}{\eta_{\mathrm{J}}}\right)^{1 / 2} c \simeq 0.447 c\left(\frac{\epsilon_{\mathrm{f}}}{1}\right)^{1 / 2}\left(\frac{\epsilon_{\mathrm{r}}}{0.1}\right)^{1 / 2}\left(\frac{\eta_{\mathrm{J}}}{1}\right)^{-1 / 2}$.

Note that given we do not resolve the jet on very small scales, velocities never exceed $\sim 0.25 c$ in our simulations. As such we expect that relativistic dynamical effects would make a negligible difference.

\subsubsection{Simulated jet properties and structure}

There are a number of methods in the literature used for injecting jet energy on scales resolved in galaxy cluster simulations. Broadly speaking they either only inject kinetic energy (e.g. Dubois et al. 2010; Gaspari et al. 2011; Yang \& Reynolds 2016a,b), or some combination of momentum/kinetic energy plus thermal energy (e.g. Cattaneo \& Teyssier 2007; Li \& Bryan 2014). Each has advantages and drawbacks, which we discuss in Section 6.1. We also note that jets have been simulated in other astrophysical scenarios, such as in star formation (e.g. Federrath et al. 2014), that use similar injection techniques. As such, in this paper we consider three main types of jet energy injection, 
which we term as momentum, thermal and kinetic jets for when only momentum, momentum plus thermal energy or purely kinetic energy is injected, respectively. On resolved scales, the jet is injected into a cylinder centred on the $\mathrm{BH}$, with a variable radius, $r_{\text {Jet }}$. This radius is varied such that the cylinder contains a fixed target gas mass, $M_{\text {Jet }}$, which for all simulations presented here is set to $M_{\text {Jet }}=10^{4} \mathrm{M}_{\odot}$, although we present the impact of other jet masses in Appendix A. The cylinder is divided into two halves (north and south), each with radius, $r_{\text {Jet }}$ and height, $h_{\text {Jet }}$ (such that the total cylinder length is $2 h_{\mathrm{Jet}}$ ), which are defined to have a fixed ratio $r_{\text {Jet }} / h_{\text {Jet }}=\tan \left(\theta_{\text {Jet }} / 2\right)$, where $\theta_{\text {Jet }}$ is the jet opening angle. For simulations presented here, $r_{\text {Jet }} / h_{\text {Jet }}=3 / 2$, such that the total cylinder volume is $(4 / 3) \pi r_{\text {Jet }}^{3}$. For all three energy injection regimes, half of the jet material is injected into the cells within each half of the cylinder, weighted according to a kernel function similar to that already used in the literature (e.g. Omma et al. 2004; Cattaneo \& Teyssier 2007; Yang et al. 2012) of the form ${ }^{1}$

$W_{\mathrm{J}}(r, z) \propto \exp \left(-\frac{r^{2}}{2 r_{\mathrm{Jet}}^{2}}\right)|z|$.

The mass injected into an individual cell, $i$, is given as

$\mathrm{d} m_{i}=\frac{\dot{M}_{\mathrm{J}} \mathrm{d} t}{2} \frac{m_{\mathrm{i}} W_{\mathrm{J}}(r, z)}{M_{\text {Weight }}}$,

where $\dot{M}_{\mathrm{J}}$ is given by equation (6), $\mathrm{d} t$ is the BH timestep, $m_{i}$ is the cell mass, $M_{\text {Weight }}=\sum_{i} m_{i} W_{\mathrm{J}}(r, z)$ is the weighted sum of cell masses in the relevant half-cylinder and the factor $1 / 2$ is to account for injecting half of the jet material into each half of the cylinder. For both the momentum and thermal jets, momentum is added to cells within the cylinder following the same weighting as the mass, such that the change in momentum of an individual cell is given as

$\mathrm{d} \boldsymbol{p}_{\mathrm{i}}=\frac{\dot{\boldsymbol{p}}_{\mathrm{J}} \mathrm{d} t}{2} \frac{m_{\mathrm{i}} W_{\mathrm{J}}(r, z)}{M_{\text {Weight }}}$,

where $\dot{\boldsymbol{p}}_{\mathrm{J}}$ is given by equation (8). The injection of mass and momentum results in a change in the kinetic energy of a cell of

$\mathrm{d} E_{i}^{k i n}=\frac{\left(\boldsymbol{p}_{i, 0}+\mathrm{d} \boldsymbol{p}_{i}\right)^{2}}{2\left(m_{i, 0}+\mathrm{d} m_{i}\right)}-\frac{\boldsymbol{p}_{i, 0}^{2}}{2 m_{i, 0}}$.

For the thermal and kinetic jets, we also calculate the total expected energy injected into each cell as

$\mathrm{d} E_{i}^{t o t}=\frac{\dot{E}_{\mathrm{J}} \mathrm{d} t}{2} \frac{m_{\mathrm{i}} W_{\mathrm{J}}(r, z)}{M_{\text {Weight }}}$,

where $\dot{E}_{\mathrm{J}}$ is given by equation (7). Due to mass loading and momentum cancellation $\mathrm{d} E_{i}^{k i n}<\mathrm{d} E_{i}^{\text {tot }}$ and thus we correct for this in the thermal and kinetic models to ensure energy conservation. In the case of the thermal jet, we inject internal energy equal to $\mathrm{d} E_{i}^{\text {therm }}=\mathrm{d} E_{i}^{\text {tot }}-\mathrm{d} E_{i}^{k i n}$. In the case of the kinetic jet, instead of injecting momentum given by equation (12), for each cell we calculate a momentum kick of magnitude

$\left|\mathrm{d} \boldsymbol{p}_{i}\right|=\sqrt{2\left(m_{i, 0}+d m_{i}\right)\left(E_{i, 0}+d E_{i}^{t o t}\right)}-\left|\boldsymbol{p}_{i, 0}\right|$,

1 We discuss an alternative kernel weighting scheme and its impact on jet evolution in Appendix B.

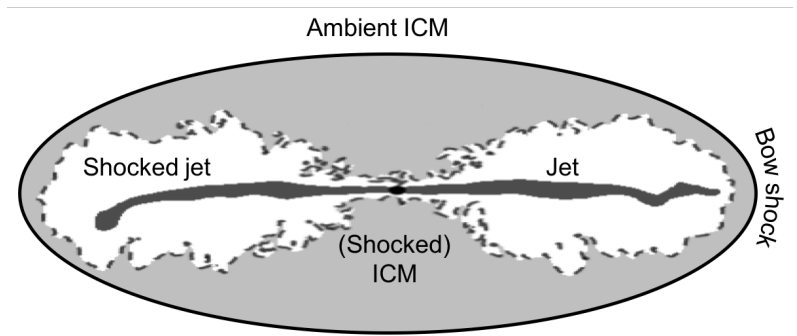

Figure 1. Schematic of the main jet components considered in this study, including the jet lobes, which consist of the jet and shocked jet material, the (shocked) ICM region, which completes the jet cocoon, and the surrounding ambient ICM.

which is added to the current momentum of the cell, along the direction of the jet. A subtlety to note here is that the final momentum of the cell will only equal $\sqrt{2\left(m_{i, 0}+\mathrm{d} m_{i}\right)\left(E_{i, 0}+\mathrm{d} E_{i}^{t o t}\right)}$, if the initial cell momentum vector and the jet momentum vector are aligned; otherwise, momentum cancellation will still result in a small loss of kinetic energy. We correct for any such loss by injecting additional thermal energy to ensure total energy conservation.

The vicinity of the jet can be split into four main regions, as illustrated in Fig. 1, which provides a schematic of the typical jet environment. We will refer throughout this paper to the regions as the jet, shocked jet material, (shocked) ICM and ambient ICM. The first three of these make up the jet cocoon. During all simulations, jet material is tracked using a tracer field, where each cell tracks the jet material mass, $m_{\mathrm{J}}$, within the cell and a jet mass fraction parameter, $f_{\mathrm{J}}=m_{\mathrm{J}} / m_{\text {cell }}$, where $m_{\text {cell }}$ is the total gas mass within the cell. $m_{\mathrm{J}}$ is set to $m_{\text {cell }}$ for any cell into which jet material, momentum and energy is directly injected (i.e., cells within the jet cylinder), with $m_{\mathrm{J}}$ being advected in line with the total gas mass. When considering the properties of the jet, similar to Yang \& Reynolds (2016a) we define the jet lobe material as those cells with $f_{\mathrm{J}}>0.01$ and the jet length as $\left[\max \left(z_{\mathrm{J}}\right)-\min \left(z_{\mathrm{J}}\right)\right] / 2$, where $z_{\mathrm{J}}$ are the $z$ co-ordinates of jet material. We note that other works in the literature (e.g. Hardcastle \& Krause 2013; Weinberger et al. 2017) use a threshold of $f_{\mathrm{J}}>0.001$. While this can impact the inferred energy content of the jet lobes (see Section 3.3.2), it has a negligible effect on the measured jet length, which is rather insensitive to the chosen value of $f_{\mathrm{J}}$.

\subsubsection{Super-Lagrangian refinement technique}

The simulations presented in this study rely heavily upon the recently published Super-Lagrangian refinement (SLR) technique of Curtis \& Sijacki (2015). The method allows grid cells to be refined in the vicinity of a $\mathrm{BH}$ according to predefined criteria for cell sizes as a function of distance from the $\mathrm{BH}$. This results in significantly improved resolution close to the $\mathrm{BH}$ allowing for more accurate estimates of the accretion rate on to the $\mathrm{BH}$ and, as shown in this paper, the ability to model AGN feedback in the form of jets. As in Curtis \& Sijacki (2015), the SLR region has an outer radius $h_{\mathrm{BH}}$, which is defined as the region surrounding the $\mathrm{BH}$ that contains a total mass of gas cells of $n_{\mathrm{ngb}}^{\mathrm{BH}} \times m_{\text {cell }}^{\text {target }}$, where $n_{\mathrm{ngb}}^{\mathrm{BH}}$ is the number of neighbouring gas cells to the $\mathrm{BH}$ (without SLR) and is set to 32 in all runs except for resolution tests, and 
$m_{\text {cell }}^{\text {target }}$ is the target cell mass of a given simulation. In order to allow for SLR of cells around the BH to take effect, jets are only activated after $\sim 2.45 \mathrm{Myr}$ in all simulations.

Despite the SLR scheme, we found that when highdensity gas flows into the vicinity of the jet, gas cells can have masses comparable to (or in excess) of $M_{\text {Jet }}$. Therefore, to reduce the risk of under populating the jet cylinder we have implemented an additional jet refinement (JR) scheme during the jet injection process. Therefore at a radius of $\gamma r_{\text {Jet }}$, the maximum allowed cell mass, $m_{\text {cell }}^{\max }$, is equal to the target jet cylinder mass, $M_{\mathrm{Jet}}$, and decreases for smaller radii following the power law

$\frac{m_{\mathrm{cell}}^{\max }}{M_{\mathrm{Jet}}}=(\alpha-\beta)\left(\frac{r}{r_{\mathrm{Jet}}}\right)^{\kappa}+\beta$,

where

$\kappa=\ln \left(\frac{1-\beta}{\alpha-\beta}\right)-\ln \gamma$,

such that $m_{\text {cell }}^{\max }=\alpha M_{\text {Jet }}$ at $r=r_{\text {Jet }}$ and $m_{\text {cell }}^{\max }=\beta M_{\text {Jet }}$ at $r=0$. Most of our runs use $\alpha=0.01, \beta=0.001$ and $\gamma=3$, which results in the jet cylinder typically being populated by $\sim 200$ cells. Note that in the remainder of this paper, all simulations include this additional JR scheme while the jet is active.

\subsubsection{Cell draining}

During the course of simulations, mass is added both to the central $\mathrm{BH}$ as it accretes material and into the jet cylinder. In order to conserve mass, material is simultaneously removed from gas cells outside of the jet cylinder, but within $r<h_{\mathrm{BH}}$, which lie within a torus-shaped region with an opening angle $\left(\pi-\theta_{\mathrm{Jet}}\right)$, in the plane perpendicular to the jet axis. The exact method used to drain mass is presented in Curtis \& Sijacki (2015), while for the generic draining procedure, see Vogelsberger et al. (2013). In the simulations presented here, the total mass drained per timestep is given by $M_{\mathrm{drain}}=\left(\dot{M}_{\mathrm{BH}}+\dot{M}_{\mathrm{J}}\right) \mathrm{d} t$, where $\dot{M}_{\mathrm{BH}}$ and $\dot{M}_{\mathrm{J}}$ are given by equations (3) and (6), respectively. The necessary mass is removed from eligible cells, with each cell contributing a mass of $M_{\text {drain }} \times m_{\text {cell }} / M_{\text {tot }}$, where $M_{\text {tot }}$ is the total mass of eligible cells. An additional condition is imposed that cells can only contribute up to 90 per cent of their total mass.

\section{NUMERICAL IMPLEMENTATION AND VERIFICATION}

\subsection{Simulations and set-up}

In this section we perform simulations to compare different numerical parameters and jet injection techniques. Apart from the simulations in Section 3.2, all other simulations are performed within a static background potential that follows a Hernquist (1990) profile and an accompanying gas distribution that follows the same profile, except for a slightly softened core (see e.g. Sijacki \& Springel 2006b; Sijacki et al. 2007). Save for the softened core, the total enclosed mass of the system initially follows:

$M(r)=M_{200} \frac{r^{2}}{(r+a)^{2}}$, where $M_{200}=10^{14} \mathrm{M}_{\odot}, a=175.98 \mathrm{kpc}$ and the gas mass makes up $f_{\mathrm{g}}=0.18$ of the total halo mass. Except for resolution testing, the gas component, which extends out to $r=100 a$, is modelled using $10^{6}$ cells, each with a target mass of $m_{\text {cell }}^{\text {target }}=1.8 \times 10^{7} \mathrm{M}_{\odot}$. The system is initially setup in hydrostatic equilibrium and relaxed non-radiatively for $\sim 5$ Gyr to produce the initial conditions. A BH particle of mass $M_{\mathrm{BH}}=10^{9} \mathrm{M}_{\odot}$ is then added to the centre of each halo to act as the source of the SLR and JR schemes, and as the source of jet feedback.

\subsection{Jet propagation: comparison to analytical models}

Before delving into comparisons of different numerical parameters, we first consider the expected propagation of jets from analytical considerations. Analytical estimates for the evolution of jet properties have previously been outlined, for example in Begelman \& Cioffi (1989), who show that the $z$-component of the velocity of the jet cavity can be estimated by balancing the thrust of the jet, $\dot{M}_{\mathrm{J}} v_{\mathrm{J}}$, where $\dot{M}_{\mathrm{J}}$ and $v_{\mathrm{J}}$ are given by equations (6) and (9), respectively, with the force due to the ram pressure of the ambient medium $\rho_{\mathrm{ICM}} v_{\mathrm{c}}^{2} \pi r_{\mathrm{ws}}^{2}$, where $v_{\mathrm{c}}$ is the velocity with which the cocoon expands in the jet direction and $r_{\mathrm{ws}}$ is the working surface radius of the jet. Given that $\dot{p}_{\mathrm{J}}=\dot{M}_{\mathrm{J}} v_{\mathrm{J}}$, the large-scale jet velocity (for a single jet) can be calculated as

$v_{\mathrm{c}}=\left(\frac{\chi \dot{p}_{\mathrm{J}}}{2 \rho_{\mathrm{ICM}} \pi}\right)^{1 / 2} \frac{1}{r_{\mathrm{ws}}}$,

where $\chi$ is a momentum boost factor that may arise, for example, due to mass loading of the jet. Equation (19) is straightforward to solve if we assume that all of the variables are constant, giving a solution for the jet length at time $t$ of

$l_{\mathrm{c}}=\left(\frac{\chi \dot{p}_{\mathrm{J}}}{2 \rho_{\mathrm{ICM}} \pi}\right)^{1 / 2} \frac{t-t_{0}}{r_{\mathrm{ws}}}+l_{0}$,

where $t_{0}$ is the jet start time and $l_{0}$ is the initial jet length, which we set to the initial height of the jet cylinder, i.e. 262 pc. This solution is plotted in the top panel of Fig. 2 by the black curves for jets with $r_{\mathrm{ws}} / \chi^{1 / 2}=0.5$ (dashed), 1 (dot-dashed) and $1.5 \mathrm{kpc}$ (dotted). Also plotted with the filled circles in the top panel are average jet lengths measured from simulations for kinetic (blue), thermal (red) and momentum (green) jets, using a simplified set of simulations compared to our other runs. Here, we model the ICM of a constant density and do not include a background potential or additional physics such as star formation or radiative cooling. This allows a more meaningful interpretation when comparing to the analytical model. While the simulated jet lengths sit within a sensible range of values when compared to the analytical solutions, none of them follow a single analytical track. To understand this further, we consider the evolution of the jet velocity, which for constant $r_{\mathrm{ws}} / \chi^{1 / 2}$ is also constant. However, the evolution of jet length found in the simulations is well fit if we assume $r_{\mathrm{ws}} / \chi^{1 / 2}$ scales linearly with time such that the jet velocity is of the form

$v_{\mathrm{c}}=\left(\frac{\dot{p}_{\mathrm{J}}}{2 \rho_{\mathrm{ICM}} \pi}\right)^{1 / 2} \frac{1}{a t+b}$, 




Figure 2. Overview: comparison of the evolution of simulated jet lengths and velocities to analytical solutions derived from the models of Begelman \& Cioffi (1989). Simulated jets differ slightly from this simple model because the working surface radius of the jet increases with time, resulting in a decelerating cocoon expansion. Top panel: evolution of jet length. Black curves show solutions to equation (20), assuming $r_{\mathrm{ws}} / \chi^{1 / 2}$ is fixed with values of 0.5 (dashed), 1 (dot-dashed) and 1.5 (dotted) kpc. Filled circles are measured directly from idealized simulations of a kinetic (blue), thermal (red) and momentum (green) jet, using the definition of jet length given in Section 2.2.3, while the solid coloured lines show fits to the points, using equation (22). Middle panel: evolution of vertical cocoon expansion velocity. Black lines show velocities calculated using equation (19) corresponding to same $r_{\mathrm{ws}} / \chi^{1 / 2}$ values used in the top panel. The solid points show velocities calculated using the change in jet length between snapshots from the appropriate simulations while the solid-coloured lines show velocities calculated from the fits to jet length, using equation (21). Bottom panel: evolution of the estimated jet working surface radius, $r_{\mathrm{ws}} / \chi^{1 / 2}$. Black lines illustrate the values chosen for the simple analytical models. Solid points and coloured lines are estimates of $r_{\mathrm{ws}} / \chi^{1 / 2}$ from the length and velocity evolution deduced from simulations. They are calculated by plugging the velocities from corresponding points and lines in the middle panel into equation (23). where $a$ and $b$ are parameters describing the evolution of the jet working surface radius and momentum boost, $r_{\mathrm{ws}} / \chi^{1 / 2}$, and hence the length of the jet evolves as

$l_{\mathrm{c}}=\left(\frac{\dot{p}_{\mathrm{J}}}{2 \rho_{\mathrm{ICM}} \pi}\right)^{1 / 2} \frac{1}{a} \ln \left(\frac{a t+b}{a t_{0}+b}\right)+l_{0}$.

We fit the jet length evolution from the simulation with equation (22), with $a$ and $b$ set as free parameters and assume that $\dot{p}_{\mathrm{J}}$ is constant ${ }^{2}$. The fits are shown by the solid lines in the top panel of Fig. 2. The middle panel then shows the evolution of the jet velocity; the solid lines are from equation (21) using $a$ and $b$ calculated from fitting the jet length evolution, while the filled points are velocities calculated using the change in jet length between consecutive snapshots. The horizontal black lines illustrate the analytic jet velocities for $r_{\mathrm{ws}} / \chi^{1 / 2}=0.5$ (dashed), 1 (dot-dashed) or $1.5 \mathrm{kpc}$ (dotted). This panel directly shows that the simulated jet velocity generally decreases with time. The changes in velocity can be attributed to an evolution of $r_{\mathrm{ws}} / \chi^{1 / 2}$ with time.

From the velocities, one can estimate the radius of the jet working surface (with $\chi$ dependence) as

$\frac{r_{\mathrm{ws}}}{\chi^{1 / 2}}=\left(\frac{\dot{p}_{\mathrm{J}}}{2 \rho_{\mathrm{ICM}} \pi}\right)^{1 / 2} \frac{1}{v_{\mathrm{c}}}$

We have estimated the working surface radii for velocities measured from both the fits to the jet length evolution and from velocities measured between snapshots; these are plotted in the lower panel of Fig. 2 with the solid lines and coloured circles, respectively. $\chi=1$ for both the thermal and momentum-driven jets, while we estimate that it varies between 2 and 3 for the kinetic jet. Therefore, the bottom panel suggests that the working surface radius of the jet increases with time. We note that while the jet cylinder radius in these simulations generally increases with time (due to the central densities falling), we also expect the jet to broaden naturally as it propagates to larger distances, a result which has also been found by previous work (e.g. Norman et al. 1982; Lind et al. 1989; Krause \& Camenzind 2001; Krause 2003) and is the likely reason why the evolution of jet length found in our simulations differs somewhat from that predicted by simple analytical arguments (e.g. Begelman \& Cioffi 1989).

\subsection{Jet parameters - a numerical study}

\subsubsection{Different refinement schemes}

When performing astrophysical simulations, there is unfortunately not a one size fits all refinement scheme and as such one has to devise a specific scheme appropriate for the problem at hand. Here, we consider the refinement and derefinement criteria necessary to ensure that we can resolve the jet to a suitable level and ensure it is able to propagate to large scales. Without any additional refinement, the typical cell mass of $\sim 1.8 \times 10^{7} \mathrm{M}_{\odot}$ is considerably larger than $M_{\text {Jet }}$. The SLR technique (in addition to the added JR scheme) therefore allows us to inject the jet into a much smaller mass

2 Strictly speaking the $\mathrm{BH}$ accretes mass during the simulation resulting in a slight increase in $\dot{p}_{\mathrm{J}}$ over time; however, this is negligibly small $(<0.5$ per cent). 

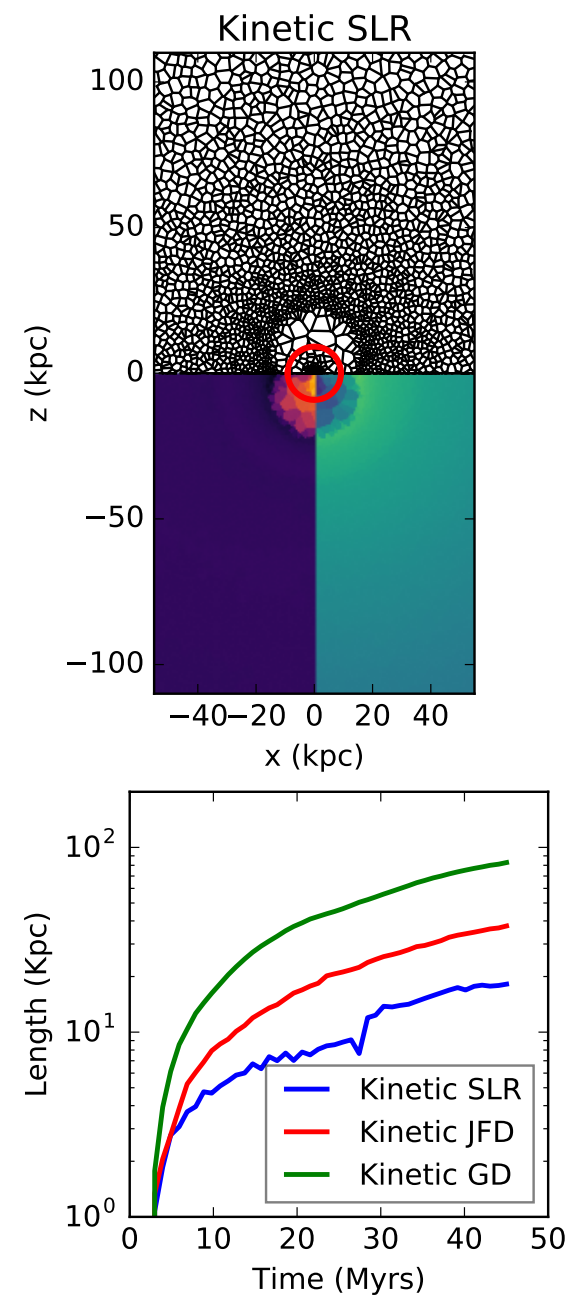
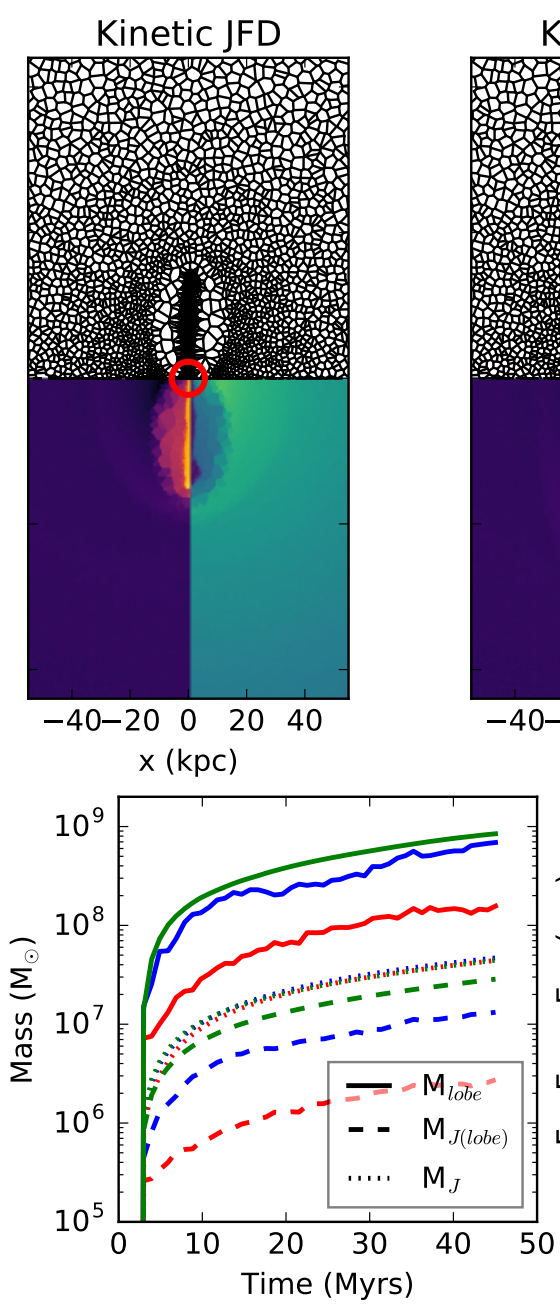
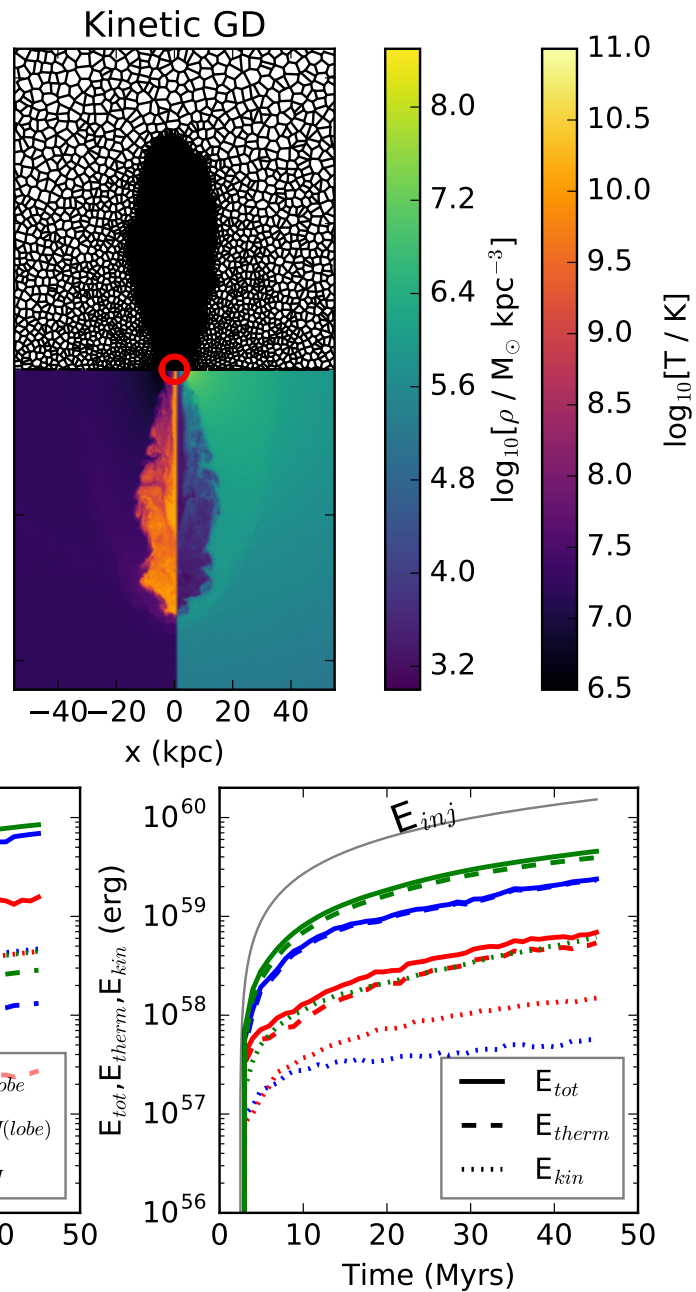

Figure 3. Overview: the impact of different refinement schemes on jet evolution. Reducing the occurrence of de-refinement allows the propagation of a jet with well-resolved interactions with the ICM. Top row: the top half of the panels show reconstructed schematics of Voronoi cell structure in the $y=0$ plane for kinetic jet runs with standard de-refinement (SLR), no de-refinement for jet material $\left[f_{\text {jet }}>0.01\right.$, jet fraction de-refinement (JFD) $]$ and gentle de-refinement (GD) from left to right respectively, at $t \simeq 45$ Myr. Note that the $\mathrm{BH}$ refinement region is indicated by the red circle, which has a radius of $h_{\mathrm{BH}}$. The bottom half of the top panels show density and temperature slices through the $y=0$ plane at $t \simeq 45 \mathrm{Myr}$ for the respective runs. Bottom row: evolution of the jet length (left-hand panel), different components of jet mass (middle panel) and different components of jet energy content (right-hand panel) for the SLR (blue), JFD (red) and GD (green) jets. For comparison, we show the total injected jet energy (equation 24) by the solid black line in the lower right-hand panel.

and hence not excessively dilute the jet properties. However, additional steps need to be taken in addition to the SLR technique outlined in Section 2.2.4. To aid the discussion of these modifications, we refer to Fig. 3, which shows in the top half of the top panels, a reconstructed schematic of the $2 \mathrm{D}$ Voronoi cell structure in the $y=0$ plane for a kinetic jet run with various de-refinement techniques (see discussion below). The figure is produced by considering the cells that exist in the $y=0$ plane and then constructing a 2D Voronoi grid based upon the mesh generating points of those cells. The left-hand panel shows the pure SLR (+JR) model outlined in Section 2.2.4. Here the level of refinement gradually reduces for cells further away from the BH until they are beyond the SLR region, defined by the smoothing length of the $\mathrm{BH}$. At this point, the cells (de-)refine based upon a mass criteria. Unmodified, this results in the jet cells produced at small radii being de-refined, as they propagate away from the BH. This is illustrated in the left-hand panel of Fig. 3, in which the cells become larger with increased distance from the BH. Indeed, cells just beyond $h_{\mathrm{BH}}$, indicated by the red circles, that are de-refined such that $m_{\text {cell }} \simeq m_{\text {target }}$, are somewhat larger than other ambient gas cells, due to their increased temperature and reduced density. Importantly, no visible jet is produced with this refinement scheme. The central panel of Fig. 3 illustrates a second de-refinement scheme in which we modify the SLR scheme such that cells can only de-refine if $f_{J}<0.01$, which we will refer to as the jet fraction de-refinement (JFD) scheme. In this case, a column of high resolution cells can be seen along the $z$-axis, which are jet cells with $f_{J}>0.01$; however, beyond these the derefinement acts in the same way as in the SLR scheme illustrated in the left-hand panel. Similarly, there is a population of large, low-density and high temperature cells that are (de)refined based on the mass criteria. Finally, the right-hand 
panel of Fig. 3 illustrates the grid structure when we employ a gentle de-refinement scheme in addition to the JFD scheme, whereby cells can only merge if gradients between neighbouring cells are suitably small. We refer to this as the gentle de-refinement (GD) scheme. This results in a jet consisting purely of high-resolution cells that can propagate to large distances and in which instabilities are not washed out. We note that as the occurrence of the de-refinement is reduced $h_{\mathrm{BH}}$ becomes smaller. We attribute this to the heating becoming less isotropic, and thus, there is an increased inflow of gas to the cluster centre perpendicular to the jet direction, and hence, the central density is increased.

The impact of the different (de-)refinement schemes on the physical properties of the jet is further illustrated in Fig. 3 . The bottom half of the top row shows density and temperature slices through the $y=0$ plane for a kinetic jet at $t \simeq 45 \mathrm{Myr}$, for the SLR, JFD and GD schemes, from left to right, respectively. The bottom row provides a quantitative overview for the evolution of jet properties, showing (from left to right) jet length, mass components and energy content for the SLR (blue), JFD (red) and GD (green) schemes. The jet properties are calculated as outlined at the end of Section 2.2.3. For comparison, the total injected jet energy is

$E_{\mathrm{Inj}}(t)=\int_{t_{0}}^{t} \dot{E}_{\mathrm{J}}\left(t^{\prime}\right) \mathrm{d} t^{\prime}$,

where $\dot{E}_{\mathrm{J}}$ is taken from equation (7) and is shown by the solid black line in the lower right-hand panel.

As already discussed, the morphology of the produced jets and their ability to propagate depends greatly on the de-refinement scheme. With strong de-refinement, the momentum (and energy) injected into the jet is diluted in more massive cells and hence the jet growth is stunted. However, as the occurrence of the de-refinement is reduced, the jets are able to travel further in the $z$-direction. Qualitatively, one can also see from the panels in the top row of Fig. 3 that stronger de-refinement can smooth out instabilities along the jet-ICM interface, potentially leading to poorly modelled mixing.

It is instructive to split the gas into different mass components, and thus, we define the total mass of jet material within cells as

$$
M_{\mathrm{J}}=\sum_{f_{\mathrm{J}}>0} f_{J} m_{\mathrm{cell}},
$$

the total mass of jet material within the jet lobes as

$$
M_{\mathrm{J},(\text { lobe })}=\sum_{f_{\mathrm{J}}>0.01} f_{J} m_{\text {cell }},
$$

and the total lobe gas mass as

$$
M_{\text {lobe }}=\sum_{f_{\mathrm{J}}>0.01} m_{\text {cell }} .
$$

The evolution of these masses is shown in the lower middle panel of Fig. 3 by the dotted, dashed and solid lines, respectively. We see that while all three runs contain very similar masses of jet material in total, the GD scheme has the highest mass in jet lobe material and retains the most jet material within the jet lobes. Further, considering the energy content (bottom right-hand panel), we find that while facilitating the longest and most massive jet, the GD scheme also facilitates the jet that retains the most injected energy $\left(\sim 30\right.$ per cent of $E_{\text {Inj }}$ in material with $\left.f_{\mathrm{J}}>0.01\right)$. On the other hand, while the jet in the SLR scheme is shorter than that of the JFD scheme, it retains more mass and energy.

Given the similar total injected jet mass between the schemes, both the jet lobe mass and the jet mass within the lobes provide an indication of the level of mixing (physical and numerical) of jet material with the ambient gas. We can see from the bottom middle panel of Fig. 3 that the JFD scheme retains the least jet material and entrained material within the jet lobes, followed by the SLR scheme and then the GD scheme. Which suggests most efficient mixing between the lobe material and ambient gas in the JFD scheme and least efficient in the GD scheme. Therefore, while we may have naively expected the strongest derefinement scheme (SLR) to result in the highest mixing, we see that the JFD scheme is most efficient at mixing jet lobe material with the ICM. We suggest that this is because of the larger surface area for mixing produced with the JFD scheme compared to the pure SLR scheme.

A further consideration is the level to which one should refine cells in the first place. In Curtis \& Sijacki (2015), the purpose of the model is to resolve the Bondi radius in order to more accurately model accretion on to the $\mathrm{BH}$ and so they set the minimum cell radius to be equal to the Bondi radius, i.e. $r_{\text {cell }}^{\min }=r_{\text {Bondi }}$. We tested the suitability of this choice and find that this provides sufficient initial resolution for jet injection. Perhaps more critically, we have tested parameters used for the additional JR scheme (see Section 2.2.4). In addition to the fiducial values of $\alpha=0.01$ and $\gamma=3$, we tried $\alpha=0.1$ with $\gamma=3$ and $\alpha=0.01$ with $\gamma=2$ and 4 . These are shown in Appendix C, with generally good convergence of jet properties.

\subsubsection{Energy and/or momentum injection}

As discussed in Section 2.2.3, we have implemented different jet models with regard to momentum and energy injection. In this section we outline how these models impact the evolution of jet properties. The top row of Fig. 4 shows density and temperature slices at $t \simeq 45 \mathrm{Myr}$ for kinetic, thermal and momentum jet runs, from left to right, respectively. The bottom row shows the evolution of jet length, mass and energy in different components for these three runs. Differences in morphology due to the different injection schemes are clearly visible in the panels in the top row. In terms of morphology, the kinetic and momentum runs are similar, with a double lobe structure. Although the size of the jet in the momentum run is much smaller because only $\sim 23$ per cent of the jet energy has actually been injected by this time (in other words, 77 per cent of the jet energy is explicitly lost due to mass loading and momentum cancellation). The jet structure in the thermal run includes an inflated central region, due to the expansion of the gas when thermal energy is injected, and is shorter than the other jets. Additionally, although the thermal jet has a greater energy content than the momentum jet, it is much shorter because the additional thermal energy results in a broader jet and hence the jet feels an increased ram pressure force acting against it, as discussed in Section 3.2.

If we consider the evolution of various mass components of these jets, we see that unlike in Fig. 3, the different energy 

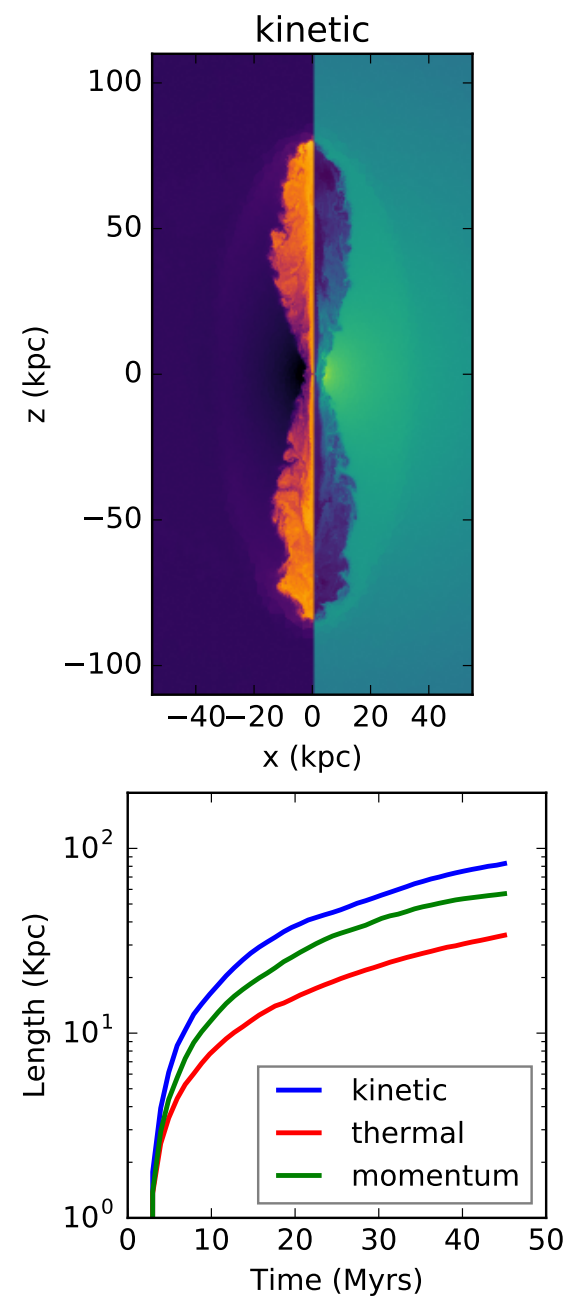
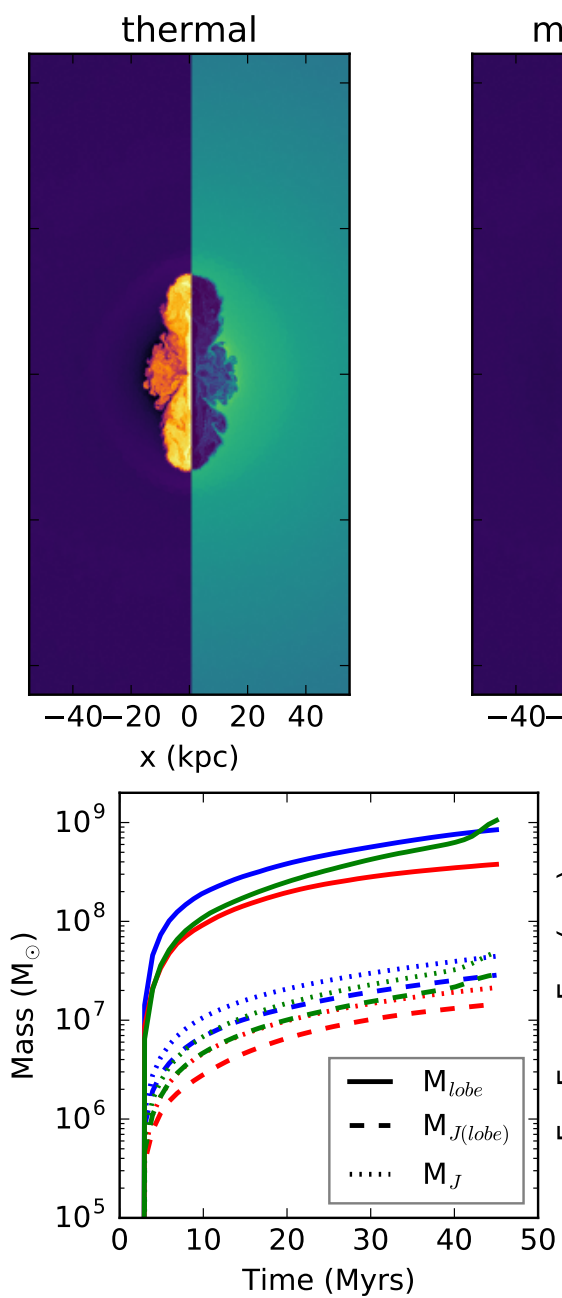
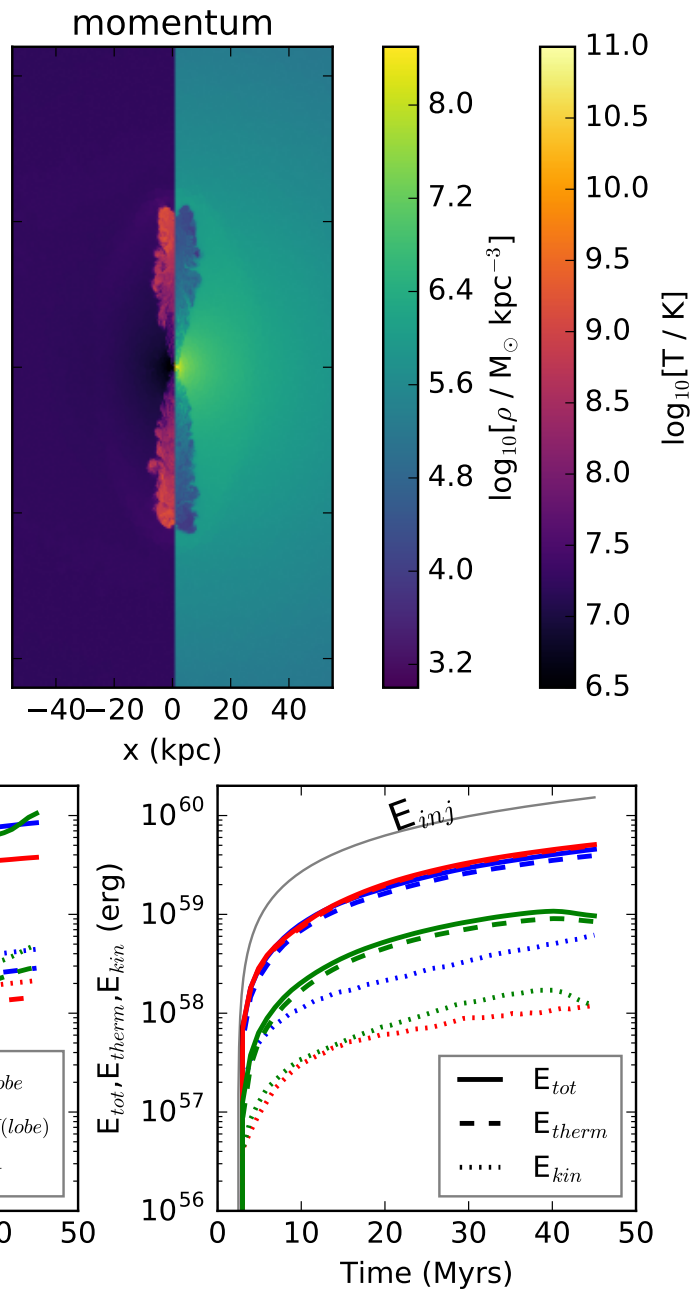

Figure 4. Overview: dependence of jet evolution on energy injection mechanism. While different mechanisms result in different morphological properties of the jet, the energy contents remain similar. Top row: density and temperature slices through the $y=0$ plane at $t \simeq 45$ Myr. Bottom row: evolution of the jet length (left-hand panel), different components of jet mass (middle panel) and different components of jet energy content(right-hand panel) for the kinetic (left-hand panel and blue curves), thermal (middle panel and red curves) and momentum (right-hand panel and green curves) runs. For comparison, we show the total injected jet energy (equation 24) by the solid black line in the lower right-hand panel.

injection techniques have different total jet material masses, $M_{\mathrm{J}}$. This can be explained by remembering that $f_{\mathrm{J}}$ is set to 1 for cells in the jet cylinder, such that the mass of jet material in those cells is set to $m_{\mathrm{J}}=m_{\text {cell }}$. Therefore the more often a cell that already contains jet material is injected with further jet material within the jet cylinder, the lower the total jet mass will be due to double counting. Therefore, one may expect that jets that are less able to quickly push cells away from the BH's location would more often inject already jetrich cells with new jet material. In line with this expectation, the total jet mass increases with the jet length, such that the kinetic jet run contains the highest mass of jet material and the thermal jet the least. Correspondingly the mass of jet material within the jet lobes and the total mass of the jet lobes follow similar trends.

Considering the bottom right-hand panel, the kinetic and thermal jet lobes contain similar amounts of energy, while the energy in the momentum run is reduced by a factor of $\sim 5$, as expected from energy conservation. Given both the momentum and thermal jets intrinsically conserve momentum, they have similar kinetic energy content, while the kinetic jet has a higher kinetic energy due to the explicit momentum boost it received $\left(\sim 2 \times \int \dot{p}_{\mathrm{J}} \mathrm{d} t\right)$. Despite this, the kinetic energy contributes $\lesssim 13.5$ per cent of the total lobe energy in all cases. Therefore, even if we assume that all of this is turbulent kinetic energy (which is unlikely due to the large bulk velocity of the jet), that places an upper limit of the turbulent energy within the lobes at the level of a few percent when compared to the total injected energy.

Specifically considering the energy budget of the kinetic jet, the total lobe energy for material with $f_{\mathrm{J}}>0.01$ is $\sim 30$ per cent of the total injected jet energy. This rises to $\sim 40$ per cent if we include gas with $f_{\mathrm{J}}>0.001$ and is consistent with other simulations of jet lobe inflation that find, depending on jet parameters, $\sim 40-60$ per cent of the energy is retained in the jet lobes (e.g. Hardcastle \& Krause 2013; English et al. 2016; Weinberger et al. 2017). In Fig. 5, we plot how the total energy budget for gas, minus the energy 

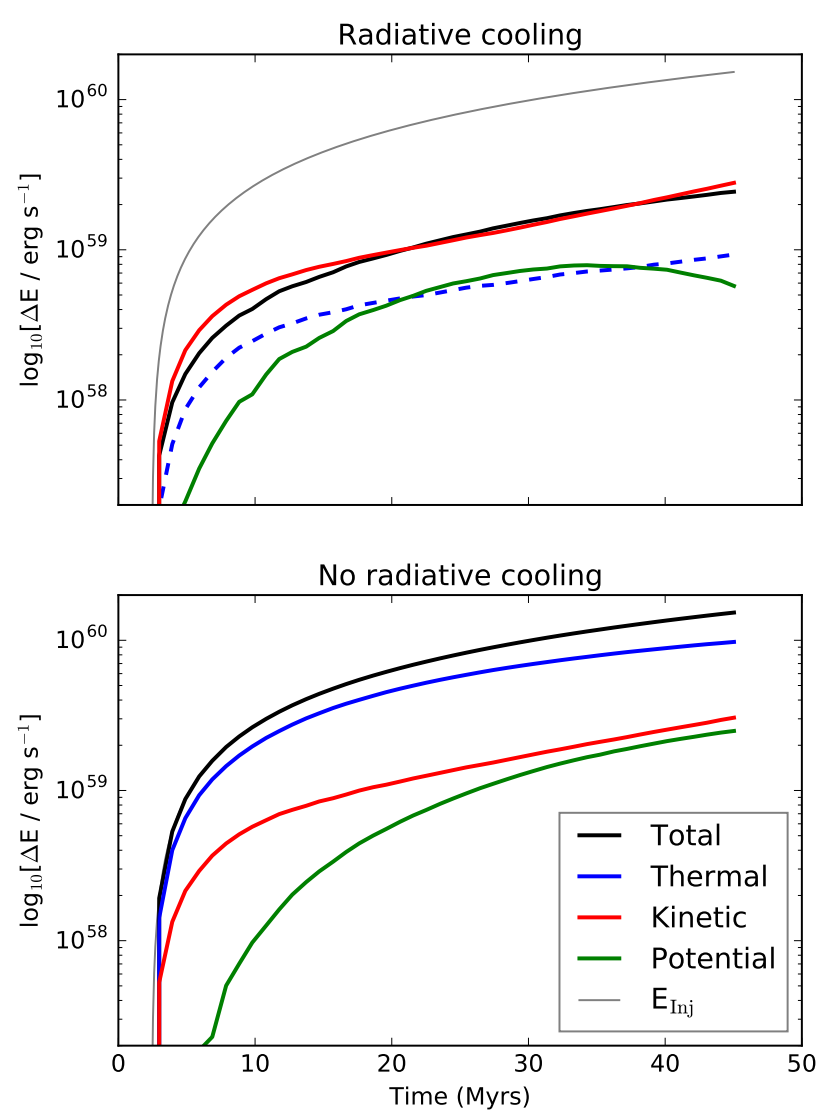

Figure 5. Overview: change in energy content within a sphere with gas mass equal to the mass of gas initially within the central $125 \mathrm{kpc}$ of the galaxy cluster, during the injection of a kinetic jet. Solid lines show energy gains while dashed lines show energy losses. Top panel: run with radiative cooling. The ICM gas loses thermal energy due to radiative cooling (blue dashes curve). However the rate of radiative cooling is reduced by the jet action, pumping thermal (blue) and kinetic (red) energy into the ICM, increasing the gravitational potential energy (green) of ICM gas lifted out of the cluster centre and leading to an overall gain in the total energy (black). Bottom panel: run without radiative cooling. The total energy (black) within the central $125 \mathrm{kpc}$ of the cluster increases in line with the energy injected by the jet (grey, $E_{\mathrm{Inj}}$ ), dominated by a gain in thermal (blue) energy, while the smaller gains in kinetic and gravitational potential energy reach similar levels to each other by $\sim 45 \mathrm{Myr}$.

content when the jet is first activated, evolves during the injection of a kinetic jet. We only consider gas within a sphere whose mass is equal to the total mass of gas $\left(\sim 3.1 \times 10^{12}\right.$ $\left.\mathrm{M}_{\odot}\right)$ within the central $125 \mathrm{kpc}$ of the cluster at $t=0$. The top panel shows the run with radiative cooling, while for comparison, the bottom panel shows a run without radiative cooling. Solid curves show gains in energy, while dashed curves show losses. In the non-radiative run, we see that the total energy (black curve) within the central region increases by the amount injected by the jet and is dominated by the thermal component (blue curve, $\simeq 0.64 \times E_{\text {Inj }}$ ), followed by the kinetic energy (red curve, $\simeq 0.2 \times E_{\text {Inj }}$ ) and gravitational potential energy components (green, $\simeq 0.16 \times E_{\text {Inj }}$ ) by $t \simeq 45$ Myr. Evidently, a sizeable portion of the injected energy goes into driving the expanding cocoon and lifting
ICM material out of the gravitational potential well of the galaxy cluster. However, without radiative cooling thermal energy dominates the budget at this point, with this energy split roughly equally between lobe and ICM gas. When radiative cooling is included (top panel), $\simeq 0.18 \times E_{\text {Inj }}$ goes into the kinetic energy component, while the total change in gravitational potential energy is much lower than in the non-radiative run, which peaks at $\simeq 0.07 \times E_{\text {Inj }}$ by $\simeq 34 \mathrm{Myr}$ but drops to $\lesssim 0.04 \times E_{\text {Inj }}$ by $t \simeq 45 \mathrm{Myr}$. The decrease in gravitational potential energy after $\simeq 34 \mathrm{Myr}$ indicates that after this point in time, gains in gravitational potential energy due to gas being pushed to larger radii are outweighed by losses in gravitational potential energy due to gas flowing back into the potential well of the cluster. Despite the significant amounts of energy being injected by the jet, there is still a net loss of thermal energy within the ICM, in the radiative run. However, globally, these losses are outweighed by the total energy injected by the jet, with the system gaining in total energy at a rate of $\simeq 0.16 \times \dot{E}_{\mathrm{J}}$.

\subsubsection{Resolution}

Finally, we consider the impact of changing the global resolution of the simulation by performing additional kinetic jet runs with $N_{\text {cell }}=10^{5}$ and $10^{7}$, giving corresponding target cell masses of $m_{\text {cell }}=1.8 \times 10^{8}$ and $1.8 \times 10^{6} \mathrm{M}_{\odot}$, respectively. The initial conditions are set-up as described in Section 3.1. Fixing the mass, opposed to the number of neighbours, into which feedback energy is injected can provide better numerical convergence (e.g. Bourne et al. 2015) and so between resolutions $h_{\mathrm{BH}}$ is set such that $\sum m_{\text {cell }}(r)=$ $5.76 \times 10^{8} \mathrm{M}_{\odot}$ for $r<h_{\mathrm{BH}}$ and $M_{\text {Jet }}=10^{4} \mathrm{M}_{\odot}$ in all runs. As in previous figures, we plot density and temperature slices in the top row of Fig. 6, with improving resolution from left to right, respectively, and in the bottom row plot the evolution of jet properties. Although the jet morphologies are quite similar, improved resolution does result in slight increases in jet length. It is also clear from the slices that larger instabilities, in the form of physically larger Kelvin-Helmholtz eddies, are observed in the lowest resolution run, which we would expect to result in increased mixing. This is born out if we compare the fraction of injected jet material that remains within the jet lobes $\left(f_{\mathrm{J}}>0.01\right)$, which decreases from $\sim 76$ per cent at the highest resolution to $\sim 53.5$ per cent in the lowest resolution, while there is also a difference of up to $\sim 23$ per cent in the jet lobe energy. This indicates that at lower resolution more of the jet material mixes with the (shocked) ICM material. The increased mixing at low resolution is of a numerical nature and therefore unphysical. However, despite these differences in mixing, the evolution of jet properties agree remarkably well and are converging with increasing resolution. We also note that if we instead consider material with $f_{\mathrm{J}}>0.001$, the differences are less stark, with the fraction of jet material within this gas only ranging between $\sim 86$ and 92 per cent between the lowest and highest resolutions and with only a negligible difference in the total energy within such material. 

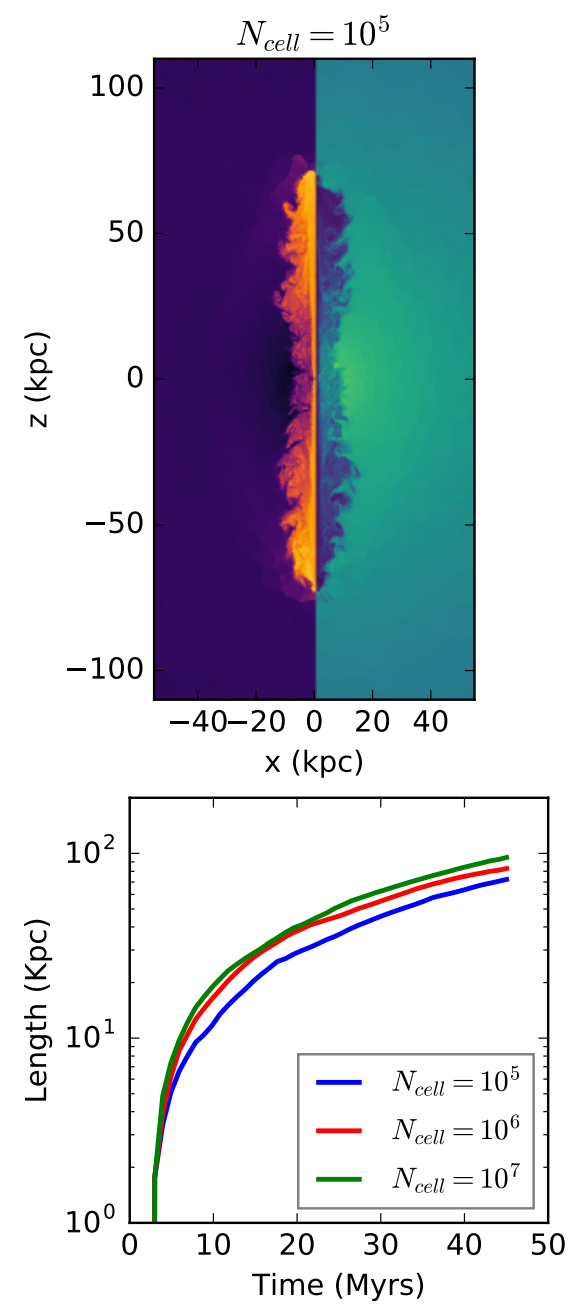
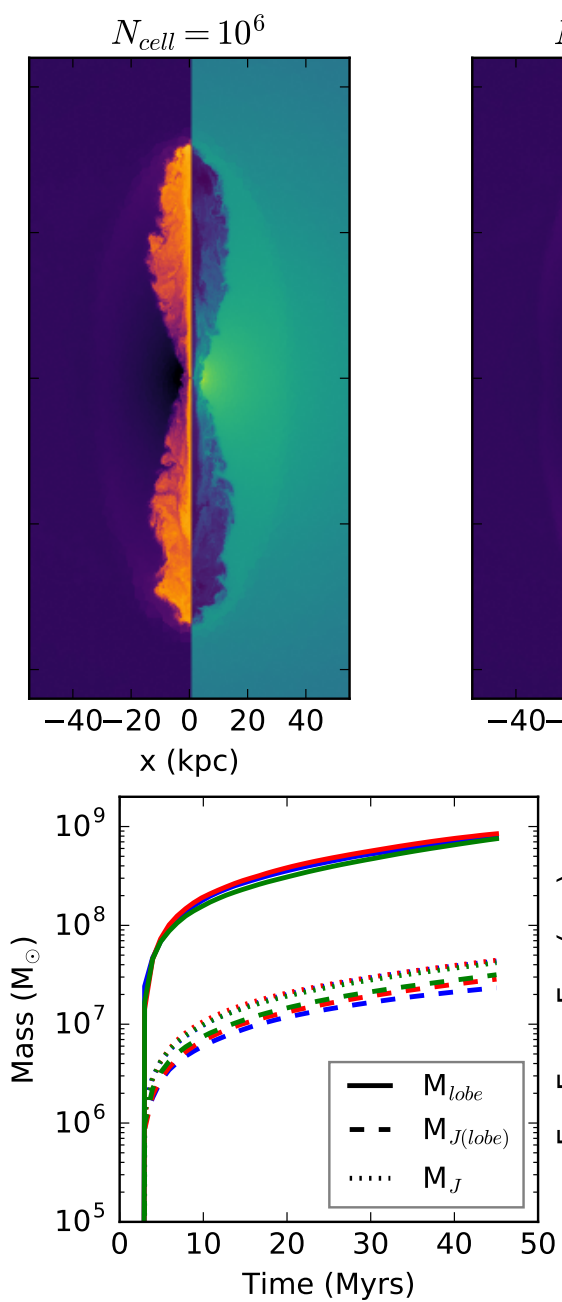
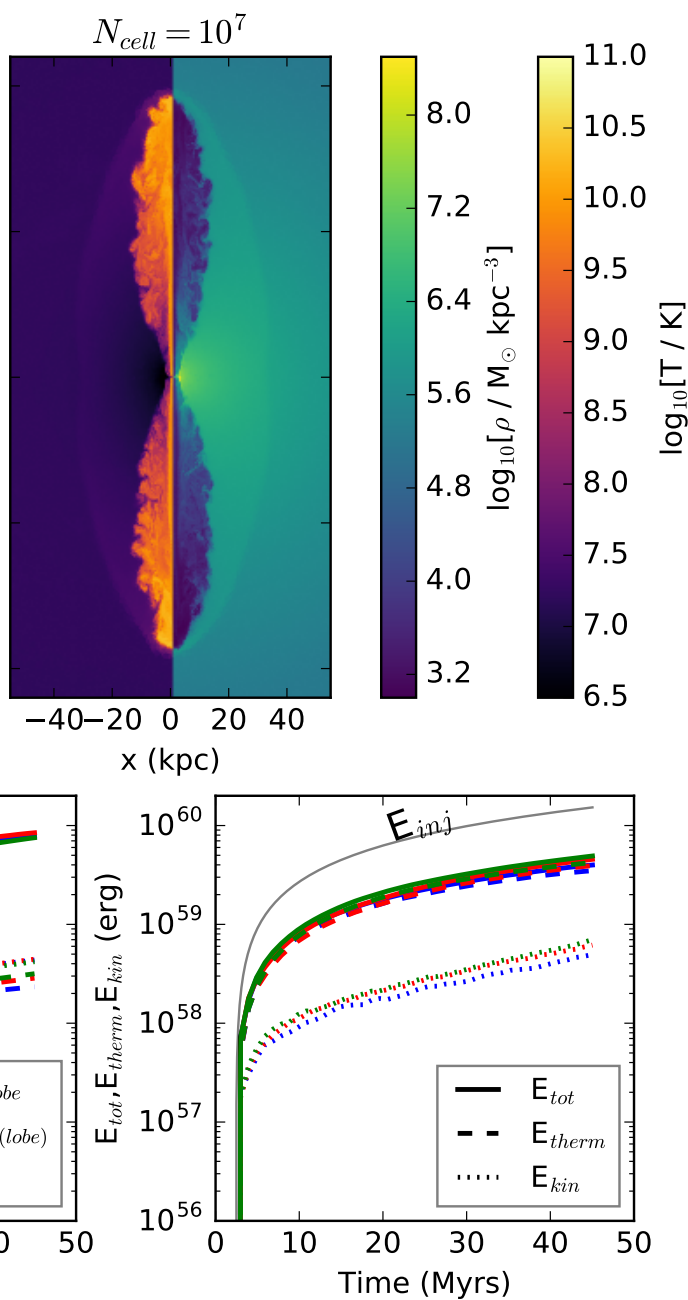

Figure 6. Overview: dependence of jet evolution (for the kinetic jet model) on global resolution of the simulation. The jet properties remain remarkably consistent over three orders of magnitude in mass resolution. Top row: density and temperature slices through the $y=0$ plane at $t \simeq 45 \mathrm{Myr}$. Bottom row: evolution of the jet length (left-hand panel), different components of jet mass (middle panel) and different components of jet energy content (right-hand panel) for the kinetic runs with $N_{\text {cell }}=10^{5}$ (left-hand panel and blue curves), $10^{6}$ (middle panel and red curves) and $10^{7}$ (right-hand panel and green curves). For comparison, we show the total injected jet energy (equation 24) by the solid black line in the lower right-hand panel.

\section{RESULTS: CAVITY INFLATION, GAS FLOWS AND PRECESSING JETS}

In this section, we consider in more detail the jet inflation process and subsequent evolution of gas flows in the vicinity of the jet. On top of this, based on the theoretical suggestion that jets need to precess in order to isotropically distribute energy to the ICM (e.g. Vernaleo \& Reynolds 2006; FalcetaGonçalves et al. 2010; Li \& Bryan 2014; Yang \& Reynolds 2016a,b) and based on observational evidence that some jets do indeed seem to precess (or at the very least move) (e.g. Dunn et al. 2006a; Martí-Vidal et al. 2011; Babul et al. 2013; Aalto et al. 2016), we also consider the impact of a precessing jet and compare the properties to those of the kinetic and thermal jets we have presented previously.

\subsection{The precessing jet}

The precessing jet runs are identical to the kinetic jet runs except that the jet axis precesses about the $z$-axis. As in
Yang \& Reynolds (2016a) we use an angle of $15^{\circ}$ and a period of $\sim 10$ Myr. The top row of Fig. 7 is similar to previous figures, showing density and temperature slices at $t \simeq 45$ Myr for kinetic, thermal and precessing jets, from left to right, respectively. The bottom row shows the evolution of jet properties. Comparison between the thermal and kinetic jets has already been made in Section 3.3.2, however, considering the precessing jet (right-hand panel), it exhibits a morphology closer to that of the thermal jet (despite being injected as a kinetic jet), but with a lower temperature, more comparable to the kinetic jet.

Initially, the precessing jet length evolution follows that of the kinetic jet (see lower left-hand panel); however, before the jet axis has completed one rotation, the length evolution flattens and tends towards that of the thermal jet. Interestingly, the precessing jet retains a similar mass in jet material within the jet lobes as the kinetic jet, although has a somewhat larger lobe mass overall, indicating that it has entrained more ICM material. Finally, the precessing 

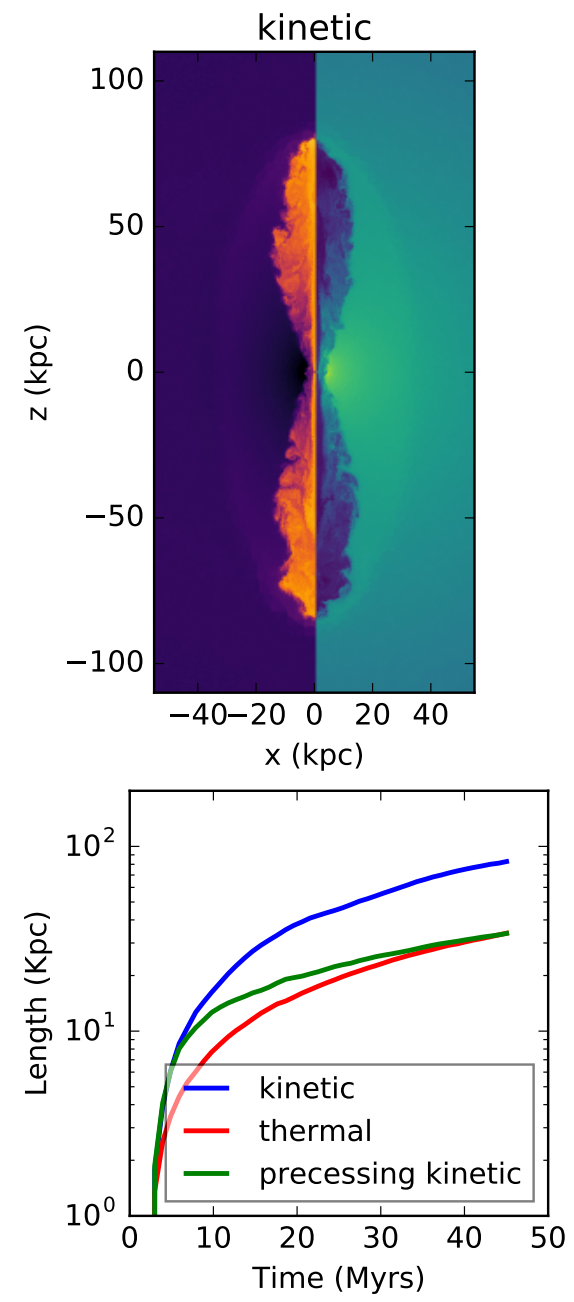
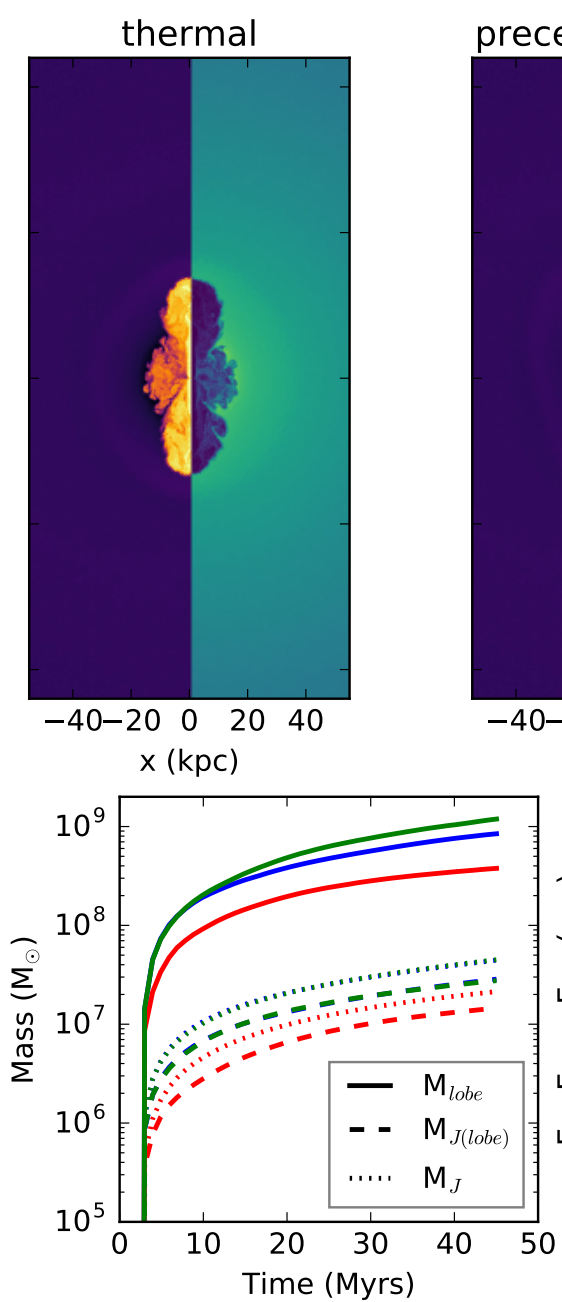
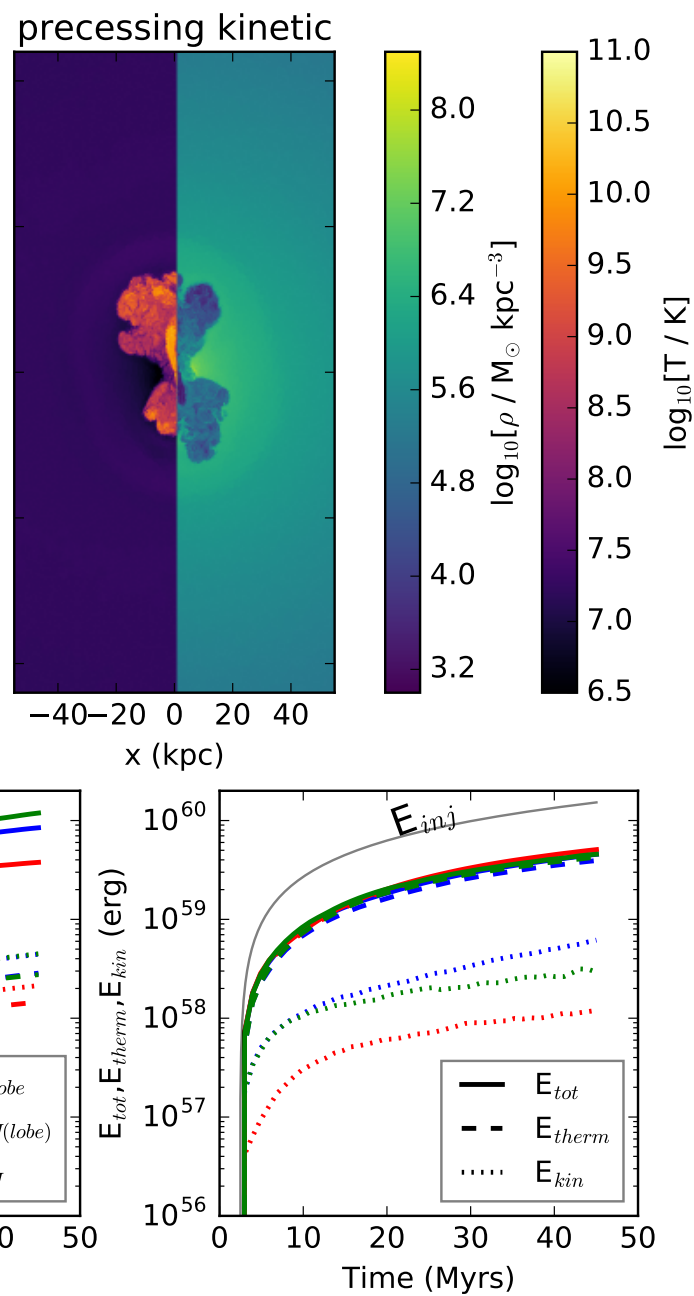

Figure 7. Overview: dependence of jet evolution when jet precession is included. The precessing jet shows a mass and energy contents similar to the kinetic jet, however a morphology closer to that of the thermal jet model. Top row: density and temperature slices through the $y=0$ plane at $t \simeq 45 \mathrm{Myr}$. Bottom row: evolution of the jet length (left-hand panel), different components of jet mass (middle panel) and different components of jet energy content (right-hand panel) for the kinetic (left-hand panel and blue curves), thermal (middle panel and red curves) and precessing (right-hand panel and green curves) jets. For comparison, we show the total injected jet energy (equation 24) by the solid black line in the lower right-hand panel.

jet lobe material retains a broadly similar energy content to the other methods. More specifically, we find that the kinetic energy content of the precessing jet sits in between the thermal and kinetic jets, indicating that the precession of the jet leads to a larger fraction of the initial kinetic energy of the jet becoming thermalized when compared to the kinetic jet.

\subsection{Jet inflation and gas flows}

Fig. 8 shows slices of various jet properties for the kinetic, thermal and precessing jet runs, from left to right, respectively, at $t \simeq 45 \mathrm{Myr}$. The top row illustrates the dominant pressure component within the jet cocoon. As discussed in Section 3.2, the pressure within the cocoon plays an important role in determining its expansion and the propagation of the jet. The total gas pressure, $P_{\text {tot }}$, is made up of a thermal $\left(P_{\text {therm }}\right)$ and a ram pressure $\left(P_{\text {ram }}\right)$ component. To determine which dominates we define the parameter:

$f_{\mathrm{P}}=\frac{P_{\text {ram }}-P_{\text {therm }}}{P_{\text {ram }}+P_{\text {therm }}}$,

such that $f_{\mathrm{P}}=1$ for ram pressure dominated gas and $f_{\mathrm{P}}=-1$ for thermal pressure dominated gas. This quantity is displayed in the top row of Fig. 8. Clearly the jet itself is dominated by ram pressure with the poles of the jet cocoon in the vicinity of the bow shock, also having a large ram pressure contribution. On the other hand, the cocoon in general and its expansion is dominated by thermal pressure. In particular, as expected from early analytical models (e.g. Blandford \& Rees 1974; Scheuer 1974; Begelman \& Cioffi 1989), the fast jet is shock heated. This results in the lobes of shocked jet material encapsulating the jet itself in which $f_{\mathrm{P}} \simeq-1$. This gas expands due to the thermal pressure, driving the growth of the cocoon and resulting in the shell of gas out to the edge of the cocoon for which $-1<f_{\mathrm{P}}<0$. We further note that similar to observations (e.g. Fabian et al. 2006; Forman et al. 2007; Croston et al. 2011; Sanders 

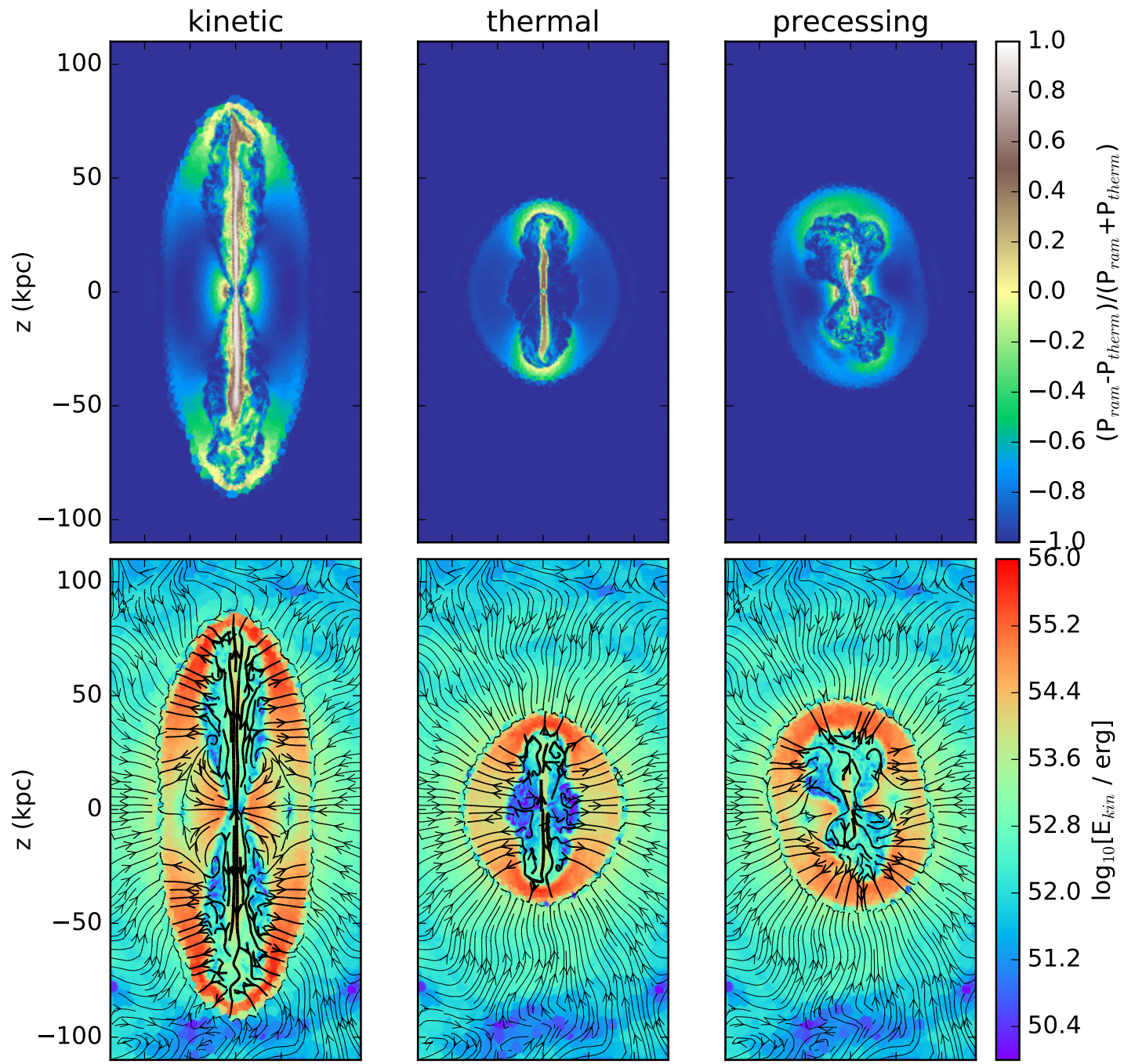

56.8

55.2

54.4

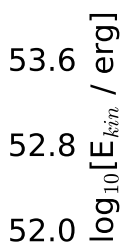

51.2

50.4
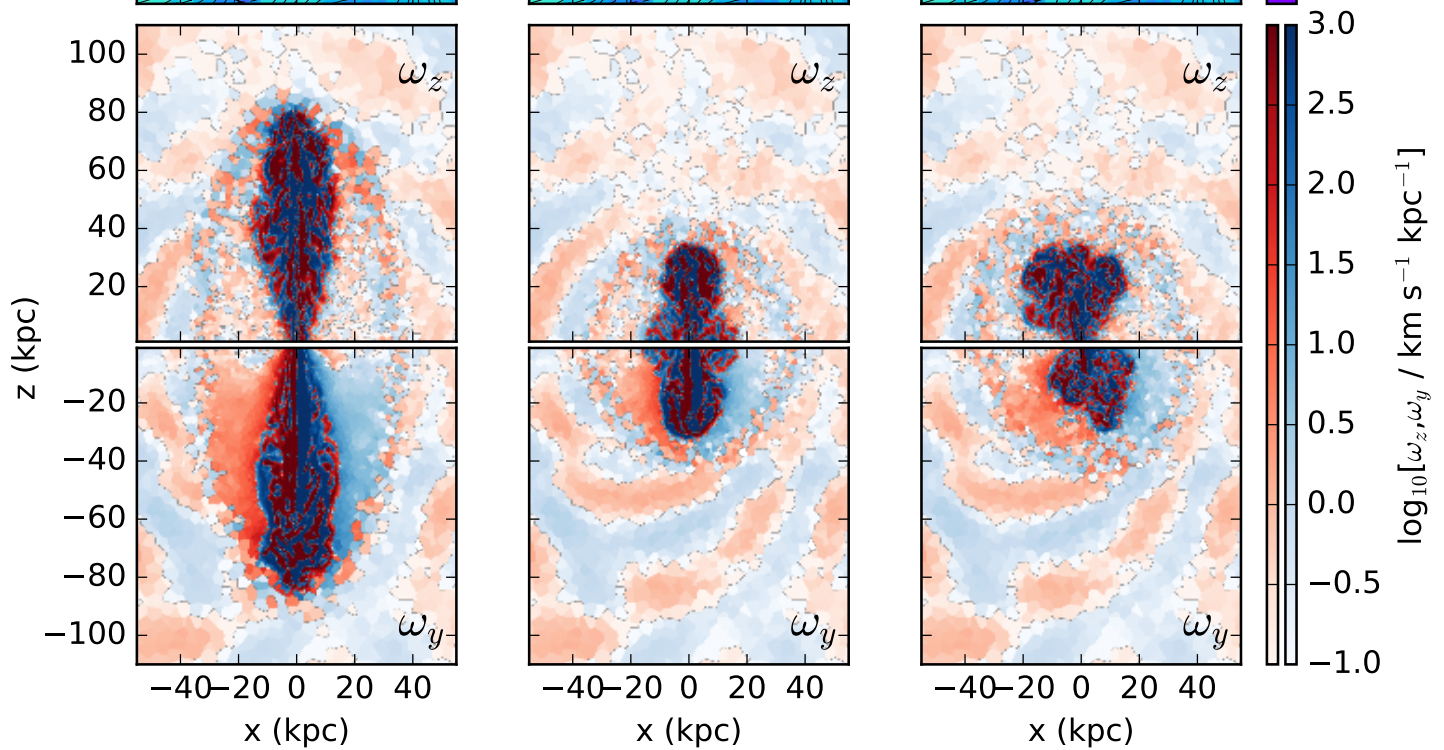

$-0.5$

$-1.0$

Figure 8. Overview: comparison of gas flows and cocoon structure properties for the kinetic, thermal and precessing jet models. All panels show slices through the $y=0$ plane at $t \simeq 45$ Myr. Ram pressure dominated jets, which have large velocities and hence high kinetic energies, thermalize through shocks and inflate thermal pressure dominated, turbulent lobes. The lobes expand and drive a massive outflowing shell that defines the boundary of the cocoon. Top row: the ratio $f_{\mathrm{P}}=\left(P_{\text {ram }}-P_{\text {therm }}\right) /\left(P_{\text {ram }}+P_{\text {therm }}\right)$, indicating the relative contributions of ram and thermal pressure to the total pressure. Middle row: the gas cell kinetic energy is shown by the colour map, while the gas velocity field is shown by the overlaid streamlines, the thickness of which vary with $|\boldsymbol{v}|$. Bottom row: component of vorticity in the $z$ - and $y$-direction, as labelled, with red and blue colours corresponding to oppositely directed vorticity vectors.

MNRAS 000, 1-31 (2017) 
et al. 2016), the expansion of the jet cocoon mainly drives only weak shocks into the ICM, predominantly at the poles of the cocoon, where the expansion velocity exceeds the ICM sound speed, $c_{\mathrm{s}, \mathrm{ICM}}$. While the cocoon expansion perpendicular to the jet direction also initially drives a shock wave into the ICM, after only a few Myr the typical radial velocity of cocoon material drops below $c_{\mathrm{s}, \mathrm{ICM}}$ and the shock wave becomes a sound wave (see also Guo et al. 2017), which propagates into the ICM with a velocity of $c_{\mathrm{s}, \mathrm{ICM}}$.

The middle row of Fig. 8 shows Voronoi cell kinetic energy slices overlaid with flow lines showing the velocity field in the $y=0$ plane. The thickness of the flow lines scales logarithmically with the gas velocity. The kinetic energy is greatest along the jet axis and in the shell of cocoon gas, which, as shown by the streamlines, is expanding into the ICM. In the case of the kinetic jet, we estimate that the kinetic energy within the expanding shell accounts for $\sim 10$ per cent of the total energy injected by the jet. A further point to note is that while the kinetic energy of the jet is dominated by high velocities, the kinetic energy of the expanding shell is dominated by its mass. With regard to the velocity field, there is a clear, elliptically shaped discontinuity at the location of the bow shocks and sound wave which outline the cocoon. The streamlines immediately beyond the cocoon boundary are inflowing, because the gas is in the cooling flow region. The streamlines also highlight interesting flow features around the base of the jet, particularly in the case of the kinetic jet. The inflation of the jet lobes and expansion of the cocoon displaces ambient ICM gas that was initially in hydrostatic equilibrium. The displaced material is pushed into a shell around the jet lobes, some of which then falls towards the centre of the cluster, converting gravitational potential energy into kinetic energy in the process. This ultimately results in the build-up of a reservoir of gas around the $\mathrm{BH}$, providing further fuel for it, but also somewhat suffocating the jets progress. Inspection of the velocity field shows gas flows within the cocoon whereby material is dragged up by the jet, circulates and returns back to the base of the jet with the inflowing material. We note that an additional class of gas flows, dubbed backflows in the literature (e.g. Antonuccio-Delogu \& Silk 2010; Cielo et al. 2014) are also observed in our simulations; however, they are susceptible to disruption by turbulent motions within the jet cocoon, especially at later times.

Finally, the injection of the jet into the ambient medium is expected to drive some level of turbulence, while the propagation of the jet may also lead to the formation of fluid instabilities. The vorticity of the gas provides a good indicator of the production of such turbulence. As such, the bottom row of Fig. 8 shows the $y$ - and $z$-components of the vorticity generated after $45 \mathrm{Myr}$, in the bottom and top half of the jet, respectively. We present these two components as they represent potentially different modes of interaction of the jet with the ICM. As discussed in Reynolds et al. (2015), in a stratified medium, the $x$ - and $y$-components of vorticity are primarily produced by $g$-modes, whereas the $z$-component of the vorticity is a product of jet-driven turbulence. It is clear from all three panels that the jets are able to generate vorticity (both $y$ - and $z$-components), with increases of up to a factor of $\sim 1000$ compared to the initial vorticity of the gas. The most significant generation of vorticity is confined to the jet and shocked jet material, indicating that the jet can readily drive turbulence and the production of $g$-modes here (see also Weinberger et al. 2017). To a lesser extent the jet is also able to drive vorticity in the (shocked) ICM gas, as most clearly illustrated for the kinetic jet in the lower left-hand panel. In particular, for $\omega_{\mathrm{y}}$, there are large regions of coherent vorticity in the (shocked) ICM material, suggesting the production of g-modes here. However, beyond the cocoon there is no noticeable increase in vorticity, suggesting that the g-modes are trapped within the cocoon boundary and that the jet is unable to drive large-scale turbulence. This is consistent with observations of the Perseus cluster by Hitomi (Hitomi Collaboration et al. 2016) and as found in other simulations (e.g. Reynolds et al. 2015; Yang \& Reynolds 2016a,b; Weinberger et al. 2017). We refer to Section 5.5 for a detailed comparison with Hitomi observations.

Additionally, while non-zero vorticity is the product of the incompressible component of the velocity field, a non-zero velocity divergence is the result of a compressible component, associated with shocks and sound waves (e.g. Reynolds et al. 2015). We can consider the relative importance of these components in different locations by considering the compressive ratio (e.g. Iapichino et al. 2011):

$r_{\mathrm{cs}}=\frac{\left\langle|\nabla \cdot \mathbf{v}|^{2}\right\rangle}{\left\langle|\nabla \cdot \mathbf{v}|^{2}\right\rangle+\left\langle|\nabla \times \mathbf{v}|^{2}\right\rangle}$,

where $\langle\ldots\rangle$ represent mass-weighted averages performed over all Voronoi cells within the region of interest. By definition, $r_{\mathrm{cs}}=1$ in regions with purely compressible flow, i.e. $\nabla \times \mathbf{v}=0$. On the other hand, $r_{\mathrm{cs}}=0$ in regions with purely incompressible flow, i.e. $\nabla \cdot \mathbf{v}=0$. Our analysis finds that for jet lobe material $\left(f_{\mathrm{J}}>0.01\right), r_{\mathrm{cs}} \simeq 0.03$ and hence is dominated by the incompressible velocity component associated with turbulence. If instead we consider outflowing cocoon material $\left(v_{\mathrm{rad}}>10 \mathrm{~km} \mathrm{~s}^{-1}\right.$ and $\left.f_{\mathrm{J}}<0.01\right)$, we find that $r_{\mathrm{cs}} \simeq 0.85$ and so the flow here is dominated by the compressible component of the velocity field associated with sound waves and shocks. Recent works have considered the role of AGN feedback in triggering star formation (e.g. Gaibler et al. 2012; Nayakshin \& Zubovas 2012; Silk 2013; Zubovas \& Bourne 2017), with the nature of the velocity field playing a potentially critical role in determining whether star formation is enhanced or inhibited (Federrath \& Klessen 2012; Federrath et al. 2017)

\subsection{How different jet models impact cluster properties}

Here, we outline the impact of jets on the gas within the cluster for the four injection techniques discussed in the previous sections. Fig. 9 shows radial profiles for different halo properties: the top row shows density, calculated using volume (solid) or emission (dotted) weighting; the middle row shows temperature, calculated using mass (solid) or emission (dotted) weighting; and the bottom row shows entropy calculated as $T \rho^{-2 / 3}$. Profiles are shown at various times for kinetic, thermal, momentum and precessing jets, from left to right, respectively. Also, shown is a black dashed curve indicating the initial, spherically averaged radial distributions. We note that given we are injecting jets with a fixed $\dot{m}_{\mathrm{J}}$, there is no self-regulation of the feedback. This was done 

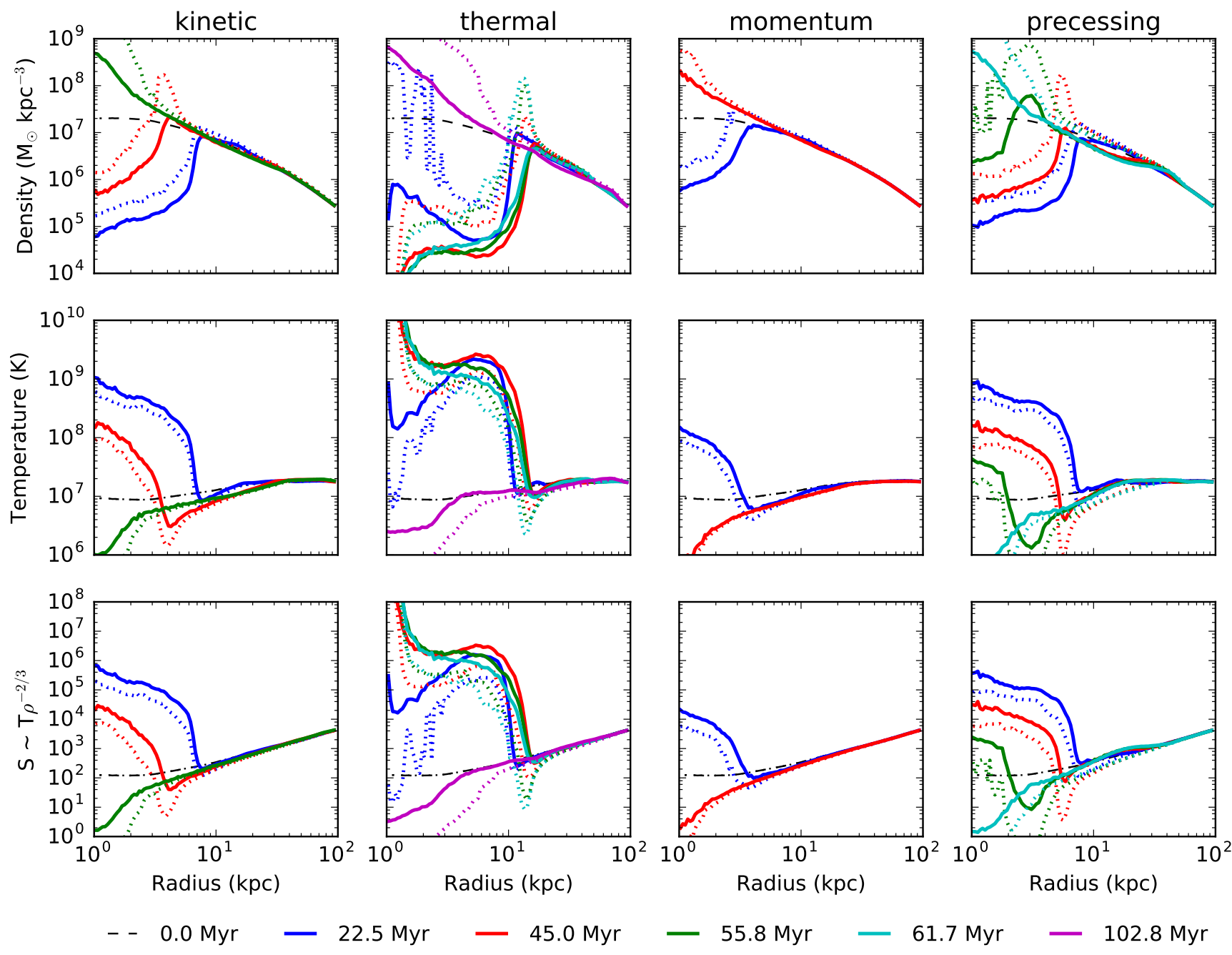

- 55.8 Myr - 61.7 Myr

Radius $(\mathrm{kpc})$

Figure 9. Overview: evolution of radial density (top row), temperature (middle row) and entropy (bottom row) profiles for (starting from the left-hand column) a kinetic, thermal, momentum and precessing kinetic jet, respectively. The density profile is calculated using either volume weighting (solid) or emission weighting (dotted), while the temperature profile is calculated using either mass weighting (solid) or emission weighting (dotted). The entropy profile is produced by combining the density and temperature profiles. The inflation of the jet cocoon results in initially reduced central densities and increased temperature and entropy profiles. However, given that these jets have a fixed $\dot{m}$, the power input does not respond to the ICM properties. Thus, eventually material can flow back into the centre of the cluster, resulting in increased central densities and reduced central temperature and entropy profiles at later times. These panels should not be used as a direct comparison to observations because of the fixed jet power, but as a diagnostic of the different jet injection methods.

intentionally to provide a clean comparison between the different jet energy injection techniques and as such Fig. 9 should be seen as a diagnostic of these techniques and not be used as a direct comparison to observed cluster profiles.

The introduction of the jet initially leads to the central density dropping sharply and the temperature and entropy increasing, particularly along the axis of the jet. However, eventually material that is displaced by the inflation of the jet cocoon flows towards the base of the jet leading to increased densities and lower temperatures and entropies at small radii. The physical size of the regions affected by the jet and when exactly material flows inwards depends upon the jet method used and how effectively it initially pushes material away from the central regions. We note that while such sharp changes in cluster radial profiles are not observed in real galaxy clusters, as mentioned previously, we deliberately inject a fixed, high power $\left(\sim 10^{45} \mathrm{erg} \mathrm{s}^{-1}\right)$ jet, in order to assess its ability to drive turbulence within the ICM. As such, we do not expect to produce profiles that match observed cluster profiles.

Comparing the different jet injection methods, we find that unsurprisingly, the least effective method is the momentum-driven jet, which impacts the smallest region with respect to the other jet models and exhibits the smallest rise in central gas temperatures. Furthermore, by $45 \mathrm{Myr}$ a central density cusp forms and the central temperature and entropy drops below the original values. On the other hand, both the kinetic and precessing jets can influence a larger 
central region and while exhibiting similar temperature profiles to each other, the precessing jet is slightly more effective at preventing gas inflow perpendicular to the jet direction. The thermal jet run can affect the largest area of gas and heats the gas to significantly higher temperatures than any of the other jet models. Further, the thermal jet is the most effective at preventing gas returning to and accumulating within the cluster centre.

The large difference between the thermal jet run and the other runs can be attributed to the direction in which the feedback is acting. In the kinetic and momentum runs, and broadly speaking in the precessing run, the feedback is primarily directed along the jet axis, with only shock and compressional heating providing more isotropic feedback. On the other hand, for the thermal jet, the internal energy, which is explicitly injected, leads to expansion of the heated gas more isotropically, thus more readily clearing the central regions.

\section{RESULTS: SUBSTRUCTURE-DRIVEN TURBULENCE AND JETS}

\subsection{The 'stirred' ICM}

Up to this point, we have presented jet feedback in a homogeneous and smooth ICM. However, realistic galaxy clusters are expected to contain substructures which stir the ICM and possibly drive turbulence (Dolag et al. 2005; Vazza et al. 2012; Gu et al. 2013; ZuHone et al. 2013; Vazza et al. 2017). As such, it is interesting to investigate how these motions impact the evolution of the jet and its cocoon. In this section, we perform simulations with a fixed $\dot{m}_{\mathrm{J}}$ kinetic jet, as described in Section 3.3.2, however, with modified initial conditions. Specifically we adopt a set-up similar to that presented in Sijacki et al. (2012) that includes ten $2 \times 10^{12}$ $\mathrm{M}_{\odot}$ subhaloes added to the relaxed initial conditions described in Section 3.1. Each subhalo consists of a live Hernquist (1990) dark matter potential consisting of 1000 dark matter particles and a gaseous component with gas fraction, $f_{\mathrm{g}}=0.17$. The subhaloes are randomly positioned between $r=625$ and $775 \mathrm{kpc}$ from the centre of the main halo, with their orbital velocities set to between 200 and $500 \mathrm{~km} \mathrm{~s}^{-1}$. This set-up is run non-radiatively and without jet feedback to allow the motions of the subhaloes to stir the ICM, inducing turbulence and bulk motions within the main halo gas that have the potential to impact jet evolution. Fig. 10 shows the column density (top), subhalo tracer mass (middle panel) and projected vorticity (bottom), respectively, at $t \simeq 1.25,2.25$ and $3.25 \mathrm{Gyr}$ (left to right, respectively). Also shown in the top panels is $r_{200}=754 \mathrm{kpc}$, indicated by the dashed white line.

As the subhaloes move through the ICM, their locations are shown at different times in the top three panels of Fig. 10. The motion of the subhaloes stirs the ICM, leading to the formation of inhomogeneous density structures and streams as gas is stripped from the subhaloes. The three snapshots provide quite different levels of perturbation and are each used as initial conditions for jet simulations. The level of stripping and subsequent mixing of the gas initially associated with the subhaloes can be seen in the middle row, which shows the line-of-sight mass of the material initially within the subhaloes. We use a tracer field, $f_{\text {sub }}=m_{\text {sub }} / m_{\text {cell }}$, similar to that used for jet material in which $f_{\text {sub }}=1$ for the cells that initially make up the subhaloes. The tracks of the stripped subhalo gas are shown by the trails in the middle row, which shows the extent to which the material permeates the central region of the cluster.

The impact of the motions of the subhaloes with regard to the velocity field and the driving of turbulence can be seen in the bottom panels, which show the projected absolute value of the gas vorticity $|\omega|$. Initially, the gas vorticity is negligible; however, as the subhaloes move through the cluster, quite significant vorticity is generated. Out of the three new initial conditions, the vorticity is strongest around $t \sim 1.25 \mathrm{Gyr}$ when it is concentrated in the centre of the cluster during the subhaloes first passage through this region (see bottom left-hand panel). As the subhaloes then leave pericentre and travel at larger radii, vorticity is produced on larger scales, but the magnitude subsides a little with time (see bottom right two panels), but is still strongest in the central regions of the cluster. The turbulence produced by the motions of the subhaloes provides extra pressure support to the cluster gas, leading to a reduced central density (for further details see Section 5.4) and the motions produced influence the evolution of jets, as we discuss now.

\subsection{Jets in a 'stirred' ICM}

Now that we have outlined the evolution of the basic properties of the ICM during the non-radiative substructure runs, we consider the different jet runs. First, Fig. 11 shows 3D contours of ambient ICM density (blue) and hot jet gas (red) for runs with and without stirring by substructure motions. From the top-left and moving clockwise, the panels show kinetic jets at $t \simeq 45 \mathrm{Myr}$ in a system where the ICM has not been stirred, and has been stirred for $\sim 1.25,2.25$ and 3.25 Gyr, respectively.

The first thing to notice is that the jets injected into a stirred ICM are somewhat disturbed and less symmetrical than the fiducial kinetic jets shown in the top-left panel, with the gas motions induced in the ICM by the subhaloes having a noticeable impact on the jet morphology. However, we find that the global jet lobe properties, such as length, mass and energy content remain remarkably similar between all of the runs. In this sense, it seems that there is no clear trend as to how concurrent substructure motions impact mixing of the lobe material, while the jet is active. Nonetheless, as we will show in Section 5.3, the motions can significantly disrupt the (shocked) ICM cocoon material and potentially aid its mixing with the ambient ICM. Although we should note that both in runs with and without substructures, the majority of jet material remains within the jet lobes while the jet is active $\left(\sim 60\right.$ per cent for $f_{\mathrm{J}}>0.01$ and $\sim 90$ per cent for $\left.f_{\mathrm{J}}>0.001\right)$. Interestingly, however, in Section 5.6 we will show that after the jet has become inactive, substructure motions can have a significant impact on the disruption and mixing of jet material when compared to runs without substructures.

\subsection{Jet inflation and gas flows}

The relative importance of ram pressure and thermal pressure, as encapsulated in the quantity $f_{\mathrm{P}}$ (see equation 25) is shown in the top row of Fig. 12. The plot is in the $y=0$ 

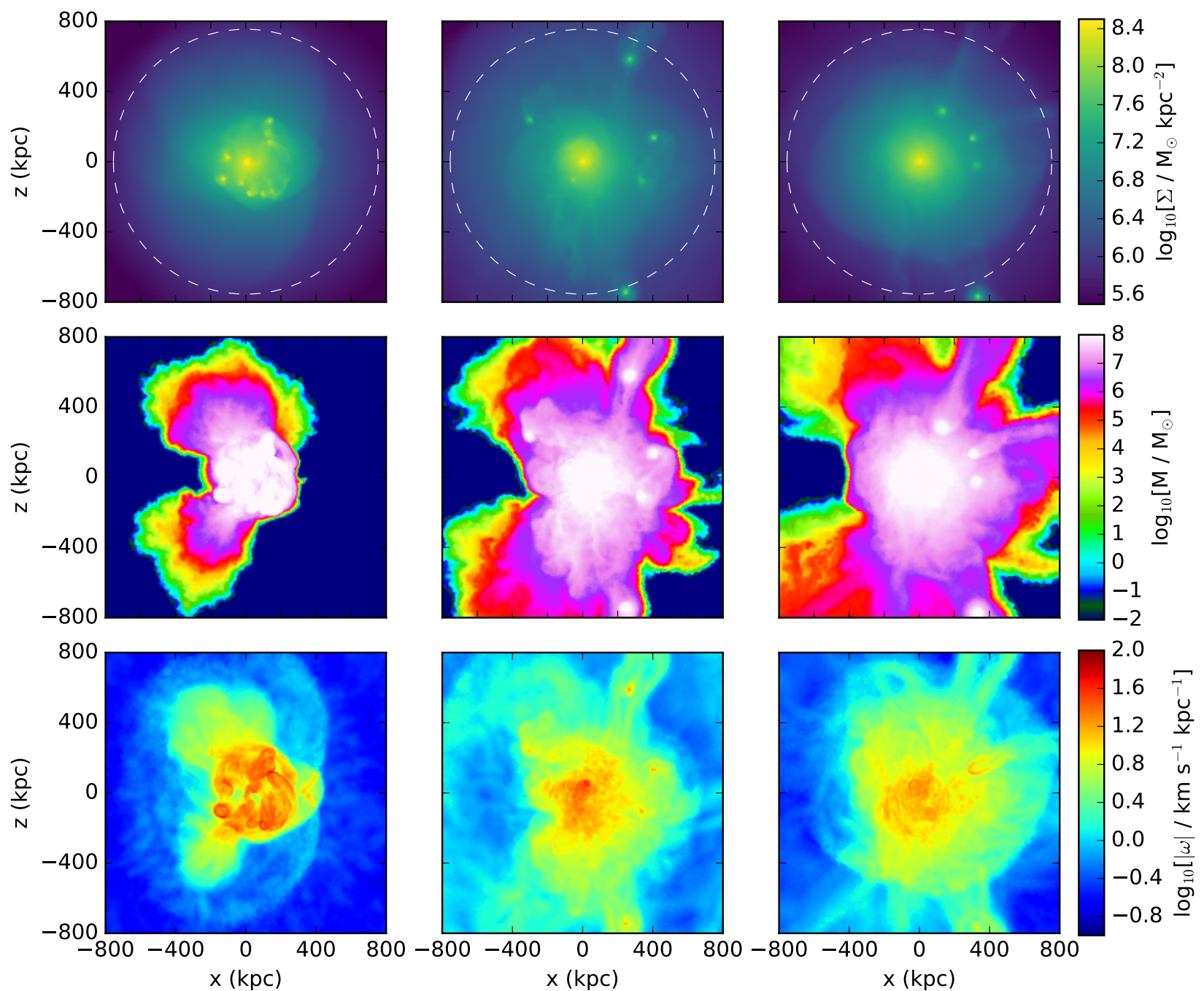

Figure 10. Overview: evolution of projected ICM properties stirred by the motions of substructures. The stirring results in density inhomogeneities, mixing of substructure and ICM material and the driving of a turbulent velocity field as illustrated by vorticity generation. The columns from left to right show projections after $\sim 1.25,2.25$ and 3.25 Gyr of stirring by substructures. Top row: evolution of the gas column density, with $r_{200}$ indicated by the dashed white circle. Middle row: evolution of the projected subhalo gas mass, calculated as the $\int\left(f_{\text {sub }} \rho_{\text {cell }}\right) \mathrm{d} z \mathrm{~d} A$ along the line of sight. Bottom row: Evolution of the projected vorticity, calculated as a mass-weighted projection of $|\omega|$.

plane at $t \simeq 45 \mathrm{Myr}$ for the jets initiated after 1.25, 2.25 and 3.25 Gyr of substructure motions. Similar to the jets shown in the top row of Fig. 8, the jet pressure is dominated by the ram pressure component, while the lobe and cocoon pressure is dominated by thermal pressure. In contrast to the fiducial run, due to the subhalo-driven motions, the gas beyond the cocoon also has a ram pressure component, which in some cases makes a contribution comparable to that of the thermal component. Further, it is interesting to consider the gas pressure components close to the base of the jet. In the top left-hand panel of Fig. 8, which shows the fiducial kinetic jet, the pressure in the plane near the base of the jet is ram pressure dominated. Combining this information with the field lines shown in the middle left-hand panel of Fig. 8, it is clear this is due to material flowing into the centre of the halo. In comparison, the central ram pressure component is reduced in the top left-hand panel of Fig. 12, while the cen- tral pressure is dominated by the thermal component in the other two top panels. This shows that gas motions driven by subhaloes help to prevent gas inflow within the innermost region of the cluster.

The impact of the gas motions produced by substructures can be seen in the middle row of Fig. 12, which like the corresponding row of Fig. 8 shows slices of the Voronoi cell kinetic energy, with the velocity field indicated by overlaid streamlines, the thickness of which scales logarithmically with the gas velocity magnitude. A clear difference between the runs with and without substructures is the additional kinetic energy beyond the jet cocoon seen in the middle row of Fig. 12, which is of a similar level to the jet cocoon material. In the fiducial jet run, shown in Fig. 8, there is a clear discontinuity in the velocity field indicating the ICM shock/sound wave at the boundary of the jet cocoon. However, this can be disrupted by the concurrent ICM 


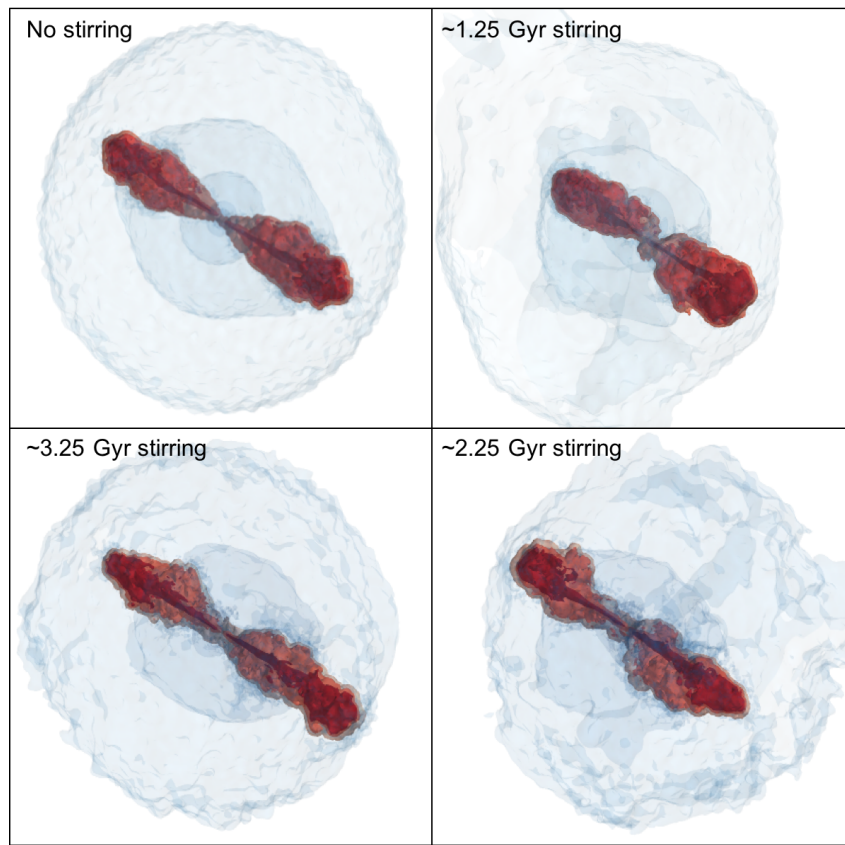

Figure 11. Overview: illustration of jet morphologies when the jet is injected into a medium that has been stirred by the motion of substructures compared to a run in which there is no stirring (top left). The figure shows that the gas motions in the ICM can distort the jet lobe morphology to become rather asymmetrical. Clockwise from top left: 3D ambient ICM density (blue) and hot gas temperature (red) contours for a jet with no stirring and jets injected into a medium that has been stirred by substructure motions for $\sim 1.25,2.25$ and $3.25 \mathrm{Gyr}$, respectively. The total jet length (end to end) in the fiducial kinetic run is $\sim 180 \mathrm{kpc}$.

velocity field in the substructure runs. In the left most panel this influence only occurs in small regions of the jet cocoon, where a vortex in the ambient gas clearly breaks into the cocoon in the top right region. However, in the other two cases, the velocity discontinuity is almost completely lost.

Finally, the bottom panels of Fig. 12 show the $z$ - and $y$ components of the vorticity in the $y=0$ plane. Similar to the corresponding panels in Fig. 8, the vorticity can be used to infer the generation of turbulence and instabilities. Vorticity in both the $y$ - and $z$-directions is produced within the jet itself, as in the fiducial kinetic jet run. The major difference observed in the runs with substructures is the increase in the pre-existing ICM vorticity, which is larger by a factor of $\sim 10-100$. Further, any jet-induced vorticity in the cocoon is subdominant with respect to the level of vorticity induced by substructures, except possibly in the bottom left-hand panel, i.e. the run with least time for substructure motions to stir the ICM, where there appears to be a slight elevation in $\omega_{\mathrm{y}}$ in the vicinity of the jet cocoon. This agrees with the behaviour observed in the middle panels, where the preexisting ICM gas flows infiltrate and even disrupt the jet cocoon.

\subsection{Evolution of cluster properties}

The next point to consider is the impact that the substructures have on the jets ability to impact the ICM properties. Fig. 13 shows the radial density (top), temperature (mid- dle panel) and entropy (bottom) profiles for the fiducial kinetic jet run (left-hand panel) followed by the haloes that had undergone 1.25, 2.25 and 3.25 Gyr of stirring by substructure motions when the jet was initiated, from left to right, respectively. Similar to Fig. 9, we show how the profiles change over time and also include the initial profiles as shown by the dashed black lines. The first thing to note is that the substructure motions have slightly altered the central gas densities and temperatures compared to the idealized cluster. Further, similar to the injection of the jets in the fiducial runs, initially the density sharply reduces, while the temperature and entropy sharply increase in the central regions. However, as also seen in the earlier runs, cold ICM material, initially displaced by the expansion of the jet cocoon, flows into the gravitational potential well of the halo and the profiles return to and somewhat exceed their initial values.

Specifically comparing to the fiducial kinetic run, it is interesting to note that the sharp peaks and troughs, at small radii, in the density, and temperature and entropy profiles, respectively, are not seen in the runs that have undergone $\sim 2.25$ and 3.35 Gyr of stirring by substructures, which instead show rather smoother profiles. The radial extent to which the AGN heats gas is somewhat larger $(\sim$ few $\mathrm{kpc}$ ) in these runs compared to the fiducial kinetic jet run. We attribute these results to the additional pressure support provided by turbulent motions as well as the ability of the substructures' motions to disrupt the jet cocoon and mix cocoon material with the ICM.

\subsection{Substructure versus jet-induced turbulence}

Given that we have introduced subhaloes to stir the ICM and induce turbulence, it is instructive to consider the $3 \mathrm{D}$ velocity power spectrum, $P_{\mathbf{v}}(k)=|\tilde{\mathbf{v}}(k)|^{2}$, where $\tilde{\mathbf{v}}(k)$ is the velocity field in Fourier space. The simulated velocity field is interpolated on to a regular grid and then following other similar procedures in the literature (e.g. Federrath et al. 2009; Vazza et al. 2009; Valdarnini 2011; Bauer \& Springel 2012; Grisdale et al. 2017), an FFT algorithm with zero padding is used to find $\tilde{\mathbf{v}}(k) . P_{\mathrm{v}}(k)$ is plotted in Fig. 14, where the top, middle and bottom panels show $k^{2} P_{\mathrm{v}}(k)$ calculated in boxes of 2,1 and $0.2 \times R_{200}$ on a side, respectively, centred on the BH. For all box sizes, we perform kernelweighted interpolation over $N_{\text {ngb }}=100$ nearest neighbours on to a $256^{3}$ grid. The power spectra on small scales (large $k$ ) can be sensitive to the number of neighbours used, although we found that the spectra are reasonably well converged, provided $N_{\text {ngb }}$ is sufficiently large. The adaptive nature of AREPO and the wide dynamical range covered in our simulations (especially after jet activity) unavoidably results in regions that are over or under sampled by our chosen grid size. This can have a minor effect upon the measured smallscale power (see e.g. Kitsionas et al. 2009; Valdarnini 2011, for further discussion), although does not impact any of our conclusions. This wide dynamic range is the primary reason for examining the power in different box sizes, while also allowing us to highlight the scales on which the jet dominates the velocity power spectrum and its relative importance to the global turbulent energy. To this end, the plot shows $k^{2} P_{\mathrm{v}}(k)$ initially and after $t \simeq 45 \mathrm{Myr}$ for the fiducial kinetic jet run (black and blue curves, respectively) and 

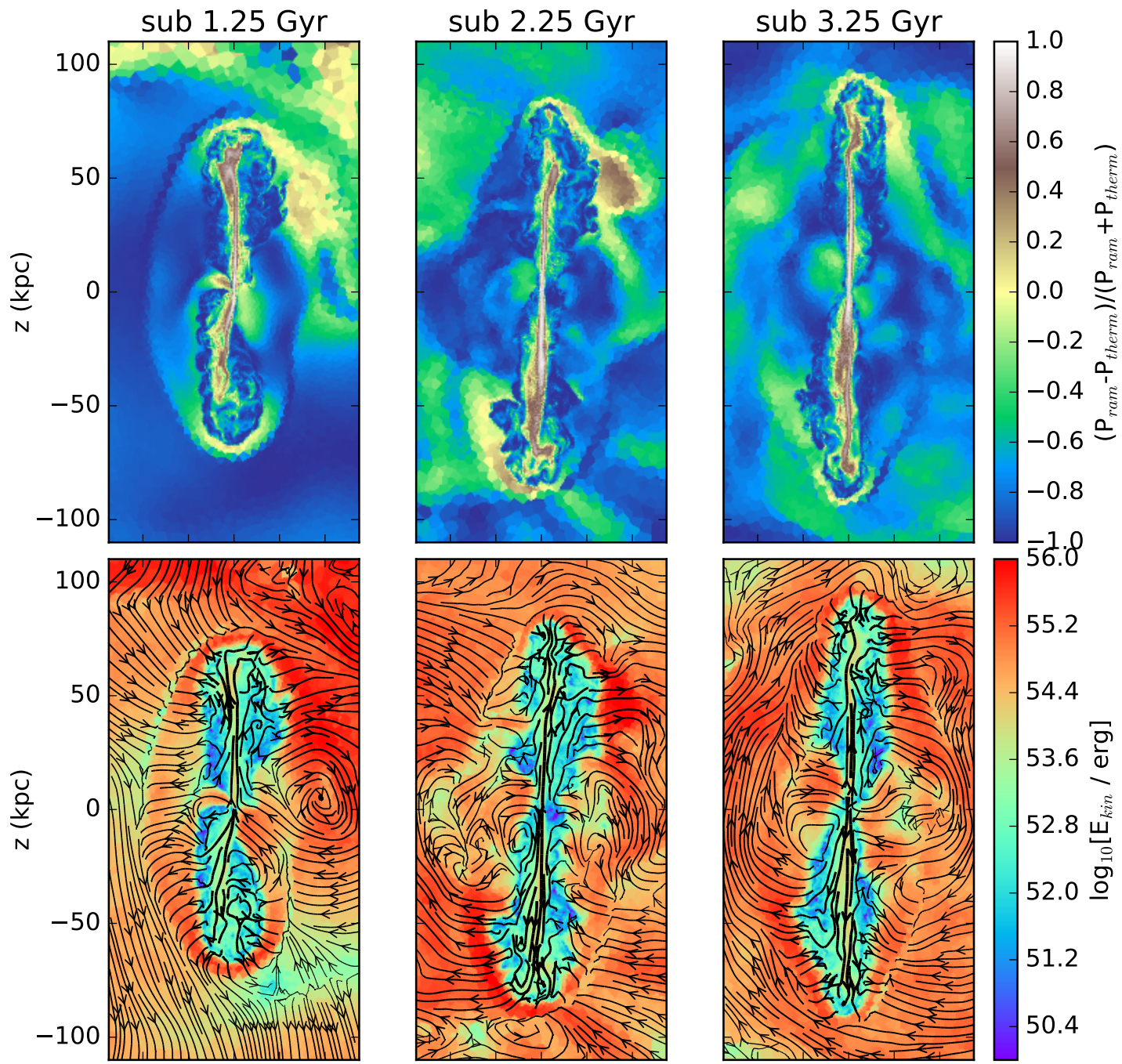

56.0

$-55.2$

54.4

53.6 ㅎํㄴ

52.8 로ำ
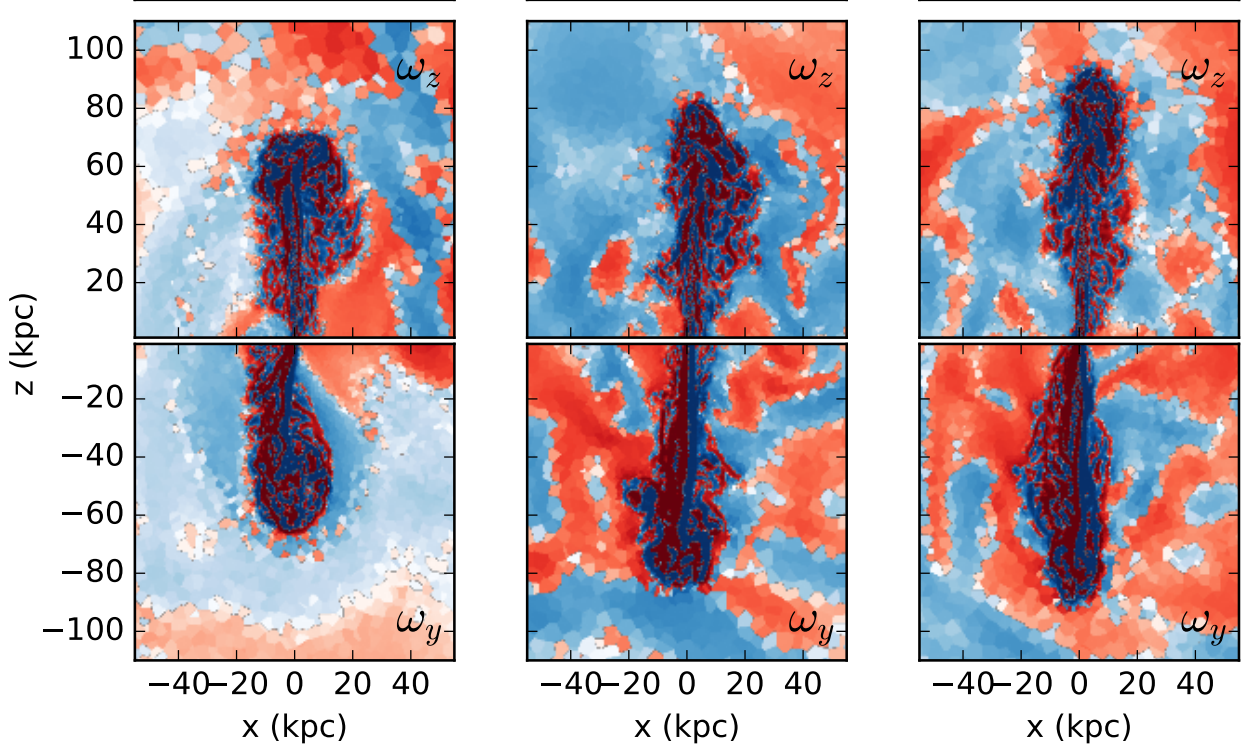

51.2

50.4

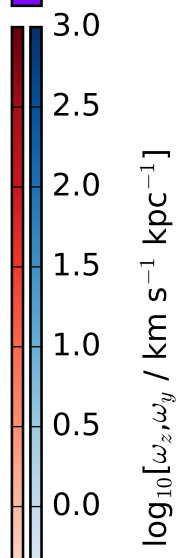

$-0.5$

$-1.0$

Figure 12. Overview: comparison of gas flows and cocoon structure properties for a kinetic jet in a cluster that has been stirred for $\sim 1.25,2.25$ and 3.25 Gyr by substructure motions. All panels show slices through the $y=0$ plane at $t \simeq 45$ Myr. Unlike in Fig. 8 , stirring by substructures results in a non-negligible level of ram pressure and vorticity within the ICM. These motions can disrupt and dominate the jet cocoon, resulting in a poorly defined outer boundary of the cocoon. Top row: the ratio $f_{\mathrm{P}}=\left(P_{\text {ram }}-P_{\text {therm }}\right) /\left(P_{\text {ram }}+P_{\text {therm }}\right)$, indicating the relative contributions of ram and thermal pressure to the total pressure. Middle row: The gas cell kinetic energy is shown by the colour map, while the gas velocity field is shown by the overlaid streamlines, the thickness of which vary with $|\boldsymbol{v}|$. Bottom row: component of vorticity in the $z$ and $y$ direction, as labelled, with red and blue colours corresponding to oppositely directed vorticity NATRrAS 000, 1-31 (2017) 

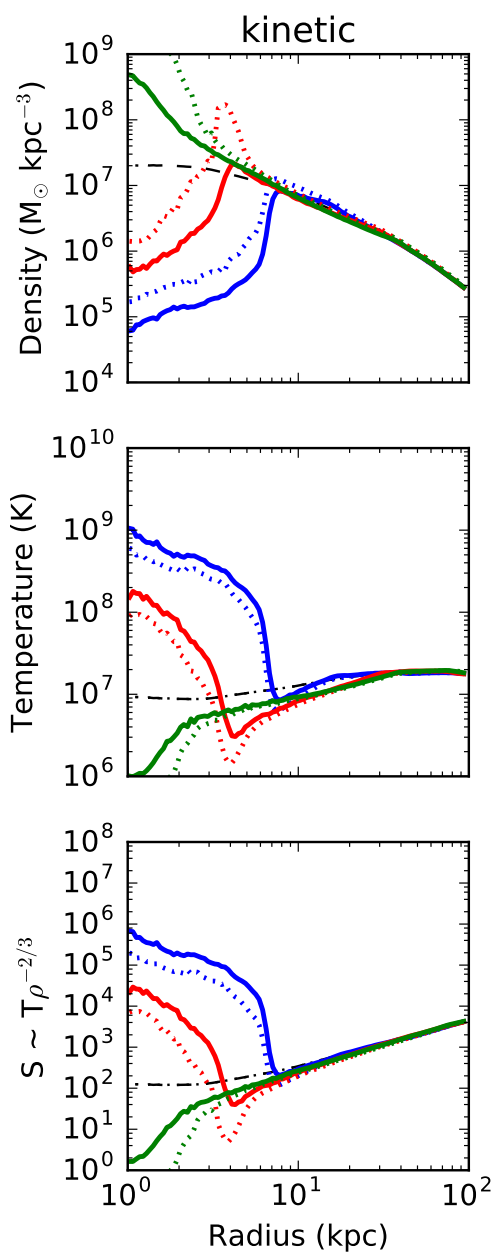

- $0.0 \mathrm{Myr}$ $22.5 \mathrm{Myr} \quad 45.0 \mathrm{Myr}$ sub 1.25
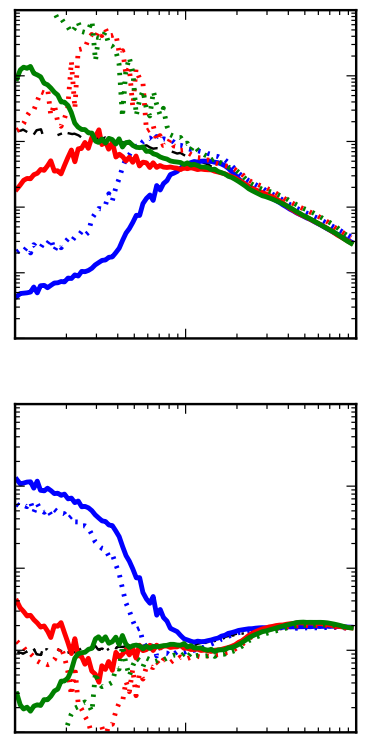

sub 2.25
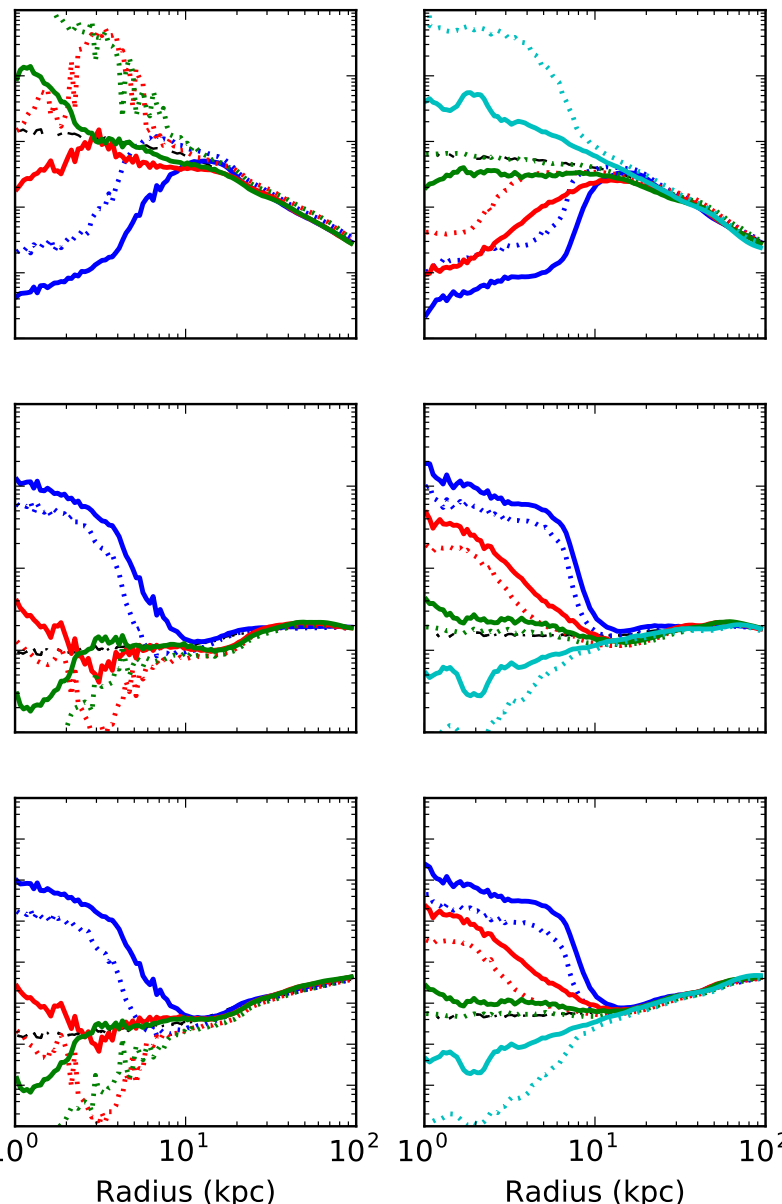

Radius (kpc)

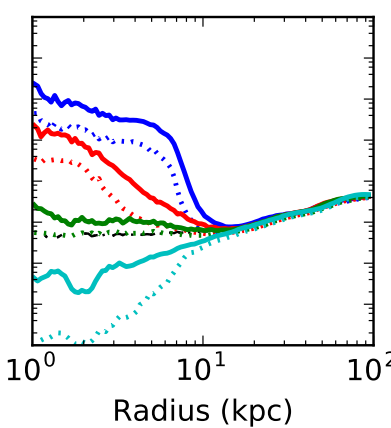

Radius (kpc) sub 3.25
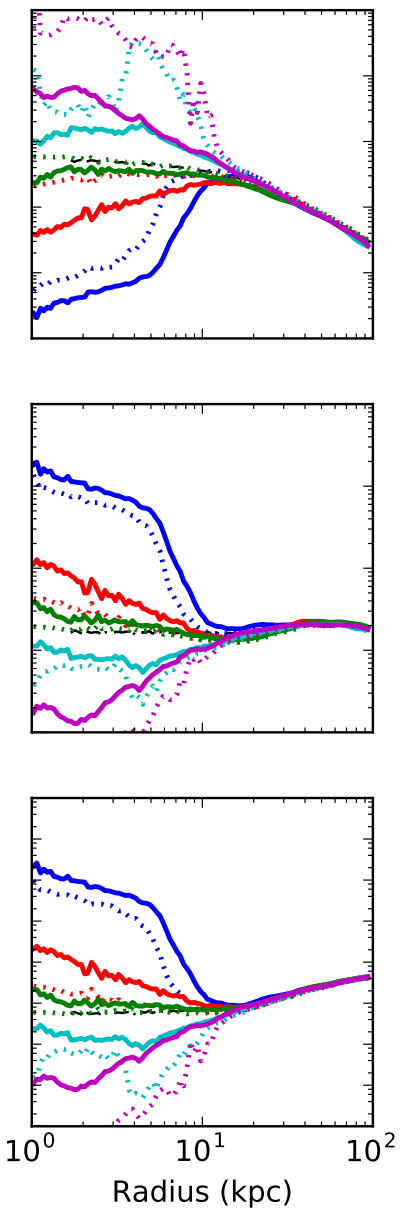

Radius (kpc)

Figure 13. Overview: evolution of radial density (top row), temperature (middle row) and entropy (bottom row) profiles for a kinetic jet in an ICM that is (starting from the left-hand column) not stirred, or stirred for $\sim 1.25,2.25$ and 3.25 Gyr, respectively. The density profile is calculated using either volume weighting (solid) or emission weighting (dotted) while the temperature profile is calculated using either mass weighting (solid) or emission weighting (dotted). The entropy profile is produced by combining the density and temperature profiles. The motions driven by the substructures provide additional pressure support to the ICM and prevent the infall of gas into the central region of the cluster. However, as in Fig. 9, given that these jets have a fixed $\dot{m}$, eventually material does flow back into the centre of the cluster.

for the run which has undergone 3.25 Gyr of substructure stirring (green and red curves, respectively). Further, the dashed curves show the contribution of jet material, defined as being cells with a jet mass fraction of $f_{\mathrm{J}}>0.01$, to the total $k^{2} P_{\mathrm{v}}(k)$.

First, we compare the initial power spectra before the jet has been inflated for the fiducial kinetic (black) and substructure (red) runs. It is clear that there is significantly more power within the run with substructures, for which $k^{2} P_{\mathrm{v}}(k)$ roughly follows the Kolmogorov turbulent power spectrum with a slope of $-5 / 3$. This is not surprising given that the motions of the subhaloes stir the ICM, generate vorticity (see Fig. 10) and drive turbulence. The next question is whether or not the jet can drive further turbulence in this system and on what scales? Starting with a box encompassing the central $\left(0.2 R_{200}\right)^{3}$ region of the cluster, shown in the bottom panel, we see that after 45 Myr the power spectra follow each other very closely and sit above the initial pre-jet power spectra for large $k$ (small physical scales, $\lesssim 30-60 \mathrm{kpc}$ ) and approach similar powers as the initial pre-jet substructure run only for smaller $k$ values (larger physical scales, $\gtrsim 60 \mathrm{kpc}$ ). It is also interesting to note that if we consider only jet lobe material (dashed curves), the power spectra follow the corresponding total power spectra almost exactly, indicating that the power in this box is dominated by the jet. Although we also highlight that this does not mean that the lobes dominate the kinetic energy, to which they contribute roughly a quarter of the budget. However, given the high vorticity (see Section 4.2) and velocity dispersion (see below) within the lobes, we do expect them to be the primary location in which the jets are able to drive turbulence. 



Figure 14. Overview: velocity power spectrum pre- and post-jet inflation for a kinetic jet in a medium that has and has not been stirred by the motions of substructures. In both cases, after the jet inflation, the power is dominated by jet material on scales smaller than the jet length. On larger scales no additional power is seen in the run without stirring, while for the run with stirring the power is dominated by the turbulence driven by the substructures motion. Coloured dashed curves indicate the power spectra when we only consider jet lobe material, while the grey dashed curve shows the slope of the Kolmogorov turbulence power law of $-5 / 3$.Top panel: power spectra calculated in a box of size $2 R_{200}$. Middle panel: power spectra calculated in a box of size $R_{200}$. Bottom row: power spectra calculated in a box of size $0.2 R_{200}$.
Next, we consider a larger box, of volume $r_{200}^{3}$, shown in the middle panel. This box could fit three jets, end to end, along the box length. In this panel, we see that the jets only dominate their corresponding power spectra for large $k$ values (on scales $\lesssim 30-60 \mathrm{kpc}$ ). The jet only run (blue curve) remains rather flat and tends towards the initial pre-jet power-spectrum (black curve) at small $k$. On the other hand, for the jet injected into the stirred ICM, the power spectrum (green curve) continues to increase with decreasing $k$, matching the pre-jet power spectrum (red curve) for $k \lesssim 0.2$ ( $\gtrsim 30 \mathrm{kpc}$ ), both of which approximately follow the $-5 / 3$ power law due to the substructure-induced turbulence. When considering only the jet lobe material, the dashed curves follow the same shape, albeit with the substructure run having slightly higher power than the run with no substructures. This shows that the jets only make a significant contribution to the velocity power spectra on small scales $(k \gtrsim 0.2, r \lesssim 30 \mathrm{kpc})$ and become subdominant on larger scales. This is particularly evident for the gas in the run with substructures, in which the substructures' motions drive turbulence and are the dominant contributor to the velocity power spectra.

Finally, the top panel shows $k^{2} P_{\mathrm{v}}(k)$ calculated over a box with sides of length $2 R_{200}$, which is considerably larger than the size of the jet. We see that the pre- and postjet power spectra in the run with substructures follow each other exactly for most of the $k$ values; that is, on these scales the substructures drive turbulence and dominate the velocity power spectra. On the other hand, the pre- and post-jet power spectra in the run without substructures follow each other exactly only below $k \simeq 0.1$, indicating that the jet is largely ineffective at impacting the velocity power spectra on the scale of this box. This is also evidenced when considering only the jet lobe material, which reaches low powers for small $k$ values. In essence, on large scales the jets have no impact on the velocity power spectra and therefore do not drive turbulence on these scales.

Additionally, we now consider the emission-weighted line-of-sight velocities and velocity dispersions within the simulated clusters. These are shown in the top and bottom panels of Fig. 15, respectively, both at our simulated resolution (first and third rows) and $20 \mathrm{kpc}$ resolution, similar to the Hitomi satellite for the Perseus cluster (second and fourth rows). From left to right, the columns show the fiducial kinetic jet run, a kinetic jet plus substructures and runs only with substructures (no jet) for the full velocity field in the third column, and only the turbulent velocity field in the fourth column. Turbulent cell velocities are calculated using a multiscale filter method similar to Vazza et al. (2012, 2017). We interpolate the cell velocities on to a regular grid, with a $3 \mathrm{kpc}$ resolution ${ }^{3}$. The velocity of each grid point can be defined as the sum of the local average velocity and a turbulent component, $\mathbf{v}_{\text {tot }}=\mathbf{v}_{\text {ave }}+\mathbf{v}_{\text {turb }}$.

3 Note that the measured turbulent velocities can vary systematically with the chosen grid resolution depending on whether the simulated velocity field is over or under sampled. Our chosen value of $3 \mathrm{kpc} \sim 0.5\left(n_{\text {cells }}\right)^{-1 / 3}$, where $n_{\text {cells }}$ is the number density of cells within the total grid volume, is a compromise to balance between over and under sampling the simulated velocity field (see e.g. Valdarnini 2011). 

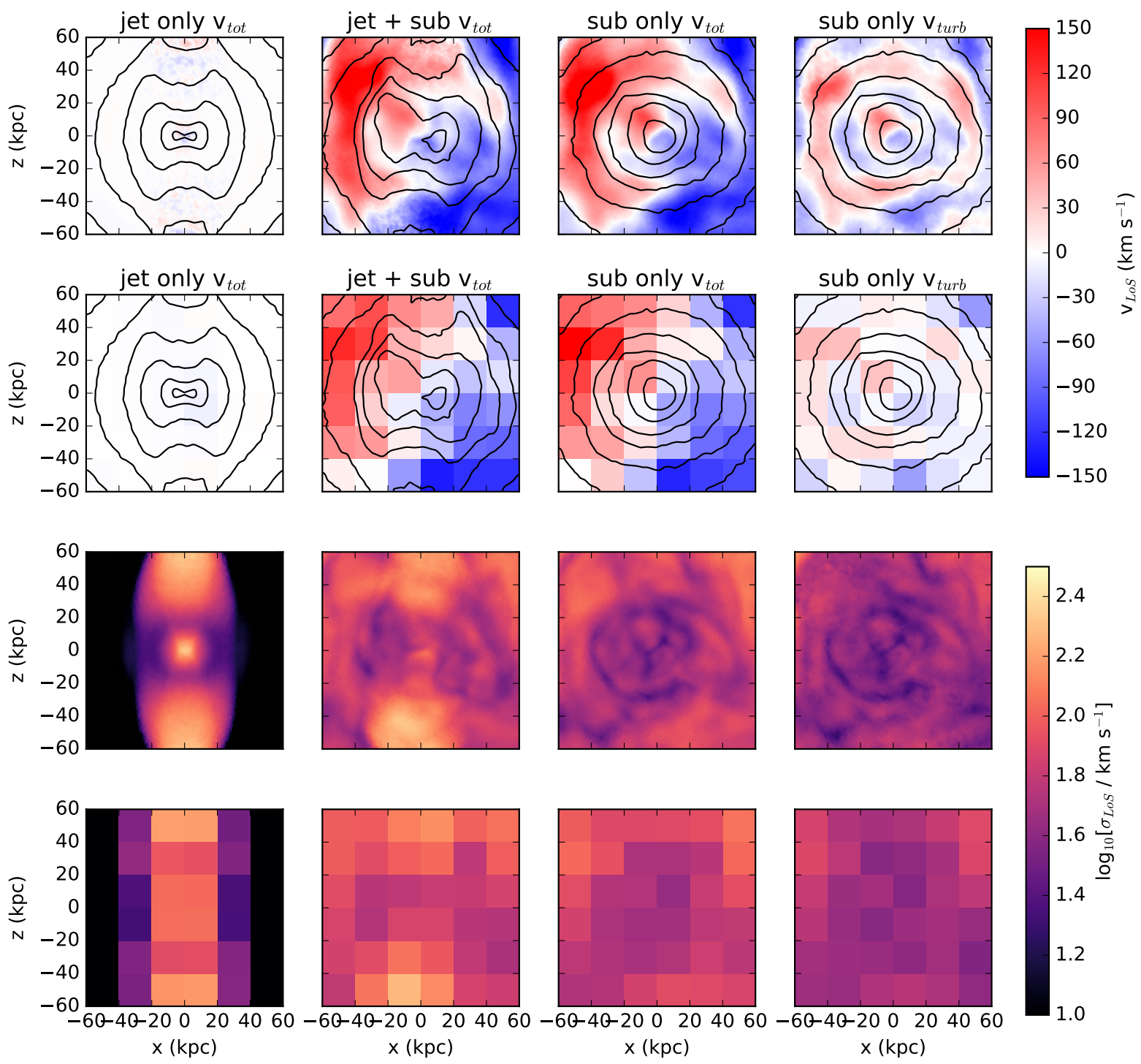

Figure 15. Overview: emission-weighted line-of-sight velocities and velocity dispersions within the central $120 \times 120 \mathrm{kpc}$ region of simulated clusters, calculated through a depth of $2 \times R_{200}$ and presented at a resolution comparable to the Hitomi Collaboration et al. (2016) observations of the Perseus cluster and at our simulated resolution. Simulations that include both a jet and stirring by the motions of substructures can qualitatively reproduce the velocity structure and disturbed X-ray emission features observed by Hitomi. From left to right, the columns show simulations that include just a jet, a jet plus substructures, only motions due to substructures and the turbulent velocities due to substructures. Upper panels: line-of-sight velocities at high resolution (first row) and Hitomi resolution (second row), overlaid with X-ray emission contours. Lower panels: line-of-sight velocity dispersion at high resolution (third row) and Hitomi resolution (fourth row).

For each grid-point the local average velocity, $\mathbf{v}_{\text {ave }}$, is calculated using a density-weighted average over neighbouring grid-points. The turbulent velocity is then calculated by subtracting $\mathbf{v}_{\text {ave }}$ from the grid-point velocity. This process is repeated, calculating $\mathbf{v}_{\text {ave }}$ over an increasing number of neighbouring grid-points, until the individual components of $\mathbf{v}_{\text {turb }}$ for a grid-point converge (to within a tolerance factor). This process is performed for each individual grid-point, providing a uniformly-spaced field of average velocities. Finally, the local average velocity for each Voronoi cell is simply taken to be the local average velocity of the nearest grid-point on which the multiscale filtering process was performed. Subsequently, the turbulent velocity of a Voronoi cell is estimated by subtracting the local average from the cell's total velocity. Finally, we include X-ray emission contours on the plots in the top row, where the X-ray luminosity is calculated using a simple Bremsstrahlung approximation (e.g. Sijacki \& Springel 2006a).

For the fiducial kinetic jet run, we see negligible lineof-sight velocities due to jet symmetry, which in this case runs along the $z$-axis and hence most of the line-of-sight velocities cancel out. We note that while viewing the jet at 

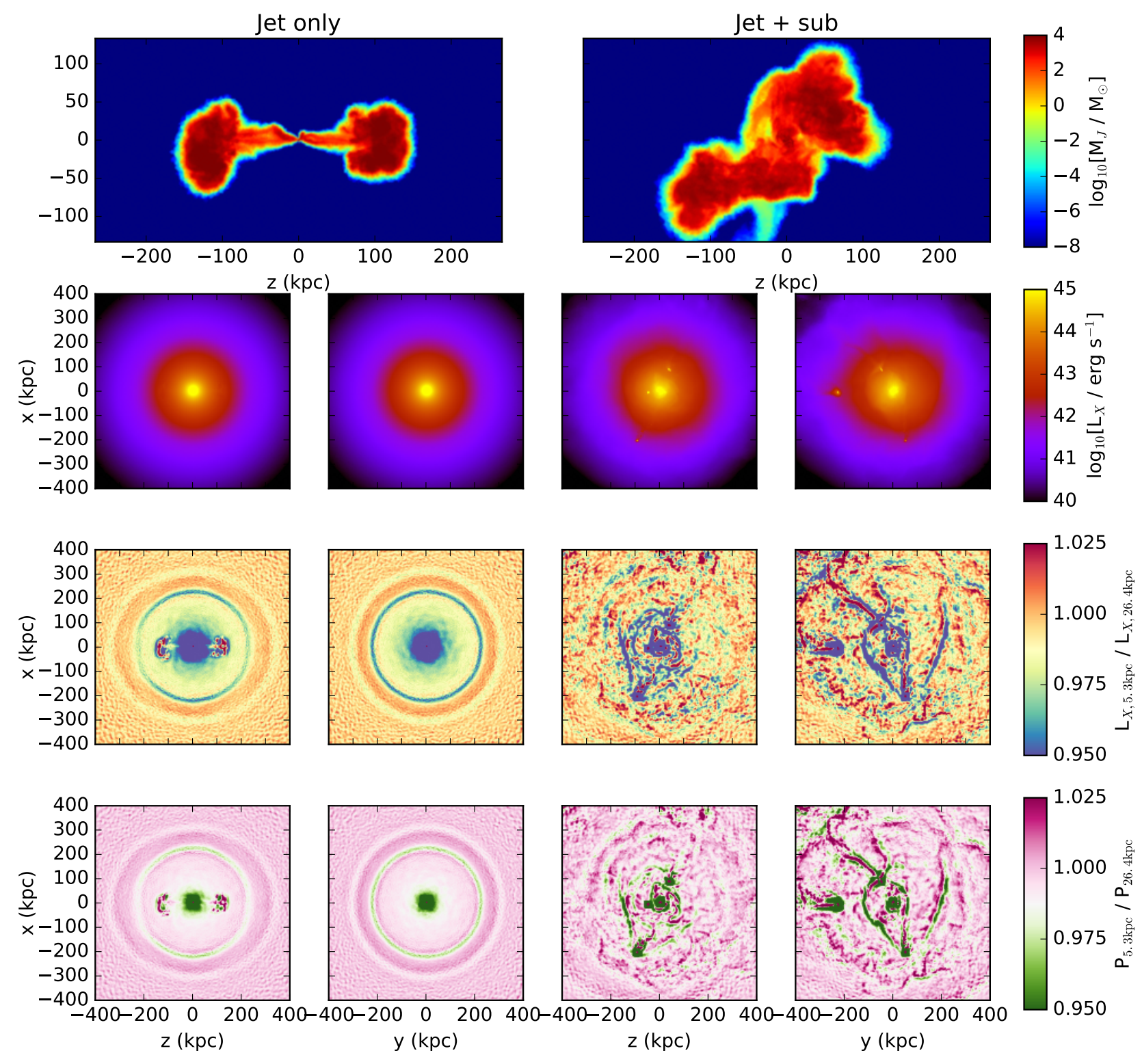

Figure 16. Overview: projected cocoon and ICM maps in the central $800 \mathrm{kpc}^{3}$ region of a galaxy cluster, $\sim 450$ Myr after jet activity has ceased. The two left most columns show a run with only a kinetic jet that is switched off after $\sim 20 \mathrm{Myr}$, while the two right most columns show a run with substructure included. Once the jet has switched off, it is possible to observe the propagation of sound (pressure) waves into the ICM. Top row: mass of jet material along the line of sight, while the jet cocoon remains axisymmetric when substructures are not included, this is clearly not the case with substructures, which can displace and disrupt the relic cavities. Second row: X-ray luminosity from the central regions. The profiles provide little indication to the existence of relic cavities. Third row: smoothed X-ray luminosity ratio maps, smoothed on different scales. Such maps highlight the positions of jet lobes as well as depressions and enhancements in the gas luminosity produced by the propagation of sound (pressure) waves. Fourth row: smooth pressure ratio maps, similar to the third row except showing features in the ICM pressure.

different angles can result in non-zero line-of-sight velocities, they do not exceed $\pm \sim 30 \mathrm{~km} \mathrm{~s}^{-1}$ at Hitomi resolution. With regard to the velocity dispersion, we see that this is dominated by the jet structure, which is in line with the jet only being able to drive significant turbulence and hence produce the observed velocity dispersion within the jet lobes (see discussion above and in Section 4.2). At face value, these results are somewhat inconsistent with the Hitomi Collaboration et al. (2016) observations of the Perseus cluster, which show a large shear velocity across the observed region and a pretty uniform, albeit low, velocity dispersion across the central region. However, if we consider our runs with substructures (which were not tuned to reproduce specific kinematic properties), both with and without a jet, we 
see similar line-of-sight velocities, between $\pm \sim 150 \mathrm{~km} \mathrm{~s}^{-1}$ with a shear produced across the positive diagonal by motions of substructures within the central cluster region. This line-of-sight velocity is dominated by the large-scale bulk motions, given that the line-of-sight velocity produced by the turbulent gas motions is significantly smaller (by a factor of 2 or more). Further, when a jet is included the substructure simulations can produce low line-of-sight velocity dispersions consistent with those observed by Hitomi in the Perseus cluster. In order to produce the distorted features observed in the X-ray luminosity contours, it is necessary to invoke both a jet, which produces the depressions in the $\mathrm{X}$ ray luminosity (compare columns 1 and 3), and substructure motions, which reduce symmetry in the emission (compare columns 1 and 2). Finally, we note that while the snapshots used in this figure were chosen for direct comparison with Fig. 14, we find that the line-of-sight kinematics found in simulations with shorter stirring times (i.e. $\sim 1.25$ and 2.25 Gyr), and for younger jets can also be consistent with the Hitomi observations with maximum velocity dispersions of $\sigma_{\text {los }} \lesssim 200 \mathrm{~km} \mathrm{~s}^{-1}$. This suggests that the gas motions observed by Hitomi within the Perseus cluster core (Hitomi Collaboration et al. 2016) are consistent with being driven by a combination of substructure motions and jet activity. We note that while our simulations only consider a single jet episode, and thus cannot definitively rule out the large-scale kinematics observed by Hitomi being driven by multiple jet outbursts, we note that we are unable to produce a largevelocity shear without including substructure motions, even if we rotate the jet along different sight lines. Further, our findings are consistent with the recent simulations of Lau et al. (2017), who found that in order to reproduce the kinematic features observed by the Hitomi satellite, a combination of cosmic accretion and jet feedback is necessary.

\subsection{Energy transport when the jets are inactive}

Up until this point we have only considered a jet which is constantly active however, it is instructive to consider the evolution of relic cavities and the ICM once a jet becomes inactive. We have performed two additional simulations in which the jet was switched off after $\sim 20 \mathrm{Myr}$ and the subsequent evolution of system was followed for a further $\sim 450$ Myr. These simulations have provided two main insights beyond those gleaned from our original set of simulations; first, we can investigate how substructure motions and the velocity field they produce can impact upon relic cavities, and secondly, we are able to investigate the propagation of sound and gravity waves within the ICM. The top row of Fig. 16 shows the mass of jet material along the line of sight, for runs with a kinetic jet with (right-hand panel) and without (left-hand panel) substructures, $\sim 450 \mathrm{Myr}$ after the jet has been switched off. When substructures are not included, the jet lobes continue to buoyantly rise through the ICM on a path dictated by the original jet direction. However, the same fate does not befall the relic cavities in the ICM stirred by substructures. While the cavities continue to buoyantly rise, they can be significantly displaced from their original trajectory, making deductions regarding original jet direction difficult. A further interesting point is that stirring by substructures can increase mixing of the jet material with the ICM by a factor of up to $\sim 3-4$, when comparing the ratio

$f_{\text {mix }}=\left(\sum_{f_{\mathrm{J}}>f_{\text {cut }- \text { off }}} m_{\text {cell }}\right) /\left(\sum_{f_{\mathrm{J}}>f_{\text {cut }- \text { off }}} f_{\mathrm{J}} m_{\text {cell }}\right)$,

for runs with and without substructures, provided that $f_{\text {cut-off }} \lesssim 10^{-5}$. For higher $f_{\text {cut-off }}$, values of $f_{\text {mix }}$ become similar between the runs, although we note that in both runs no gas exists with $f_{\mathrm{J}} \gtrsim 3 \times 10^{-3}$. Further, we find that while $\sim 89$ per cent of jet material still resides within cells with $f_{\mathrm{J}}>10^{-4}$ for the run without substructures, this drops to $\sim 59$ per cent when substructures are included.

Both pressure waves (more commonly referred to as sound waves in the literature) and gravity waves are expected to be driven by jet activity and the inflation of jet cocoons (e.g. Omma et al. 2004; Ruszkowski et al. 2004; Sijacki \& Springel 2006b,c; Sijacki et al. 2007; Reynolds et al. 2015; Guo et al. 2017). Observations appear to indicate the presence of AGN-driven sound waves within the ICM (e.g. Fabian et al. 2003, 2005b, 2017), although the location at which they deposit their energy intricately depends upon the ICM viscosity (e.g. Ruszkowski et al. 2004; Sijacki \& Springel 2006c). On the other hand, while gravity waves have been seen in simulations of jet feedback (e.g. Omma et al. 2004; Reynolds et al. 2015), it is expected that they do not carry sufficient energy to significantly impact ICM cooling (Reynolds et al. 2015; Fabian et al. 2017). In the simulations we present here, the expansion of the cocoon is initially faster than the ICM sound speed and hence drives a shock into the ICM. However, as the expansion rate of the cocoon material slows, the shock wave broadens into a sound wave that propagates into the ICM (see also Guo et al. 2017). The sound wave is most readily detectable once it has had time to propagate to large radii and after the jet has become inactive. The location and shape of this sound wave $\sim 450$ Myr after the jet has been switched off can be seen in the bottom two rows of Fig. 16. The ratio of projected Xray luminosity (third row) and pressure (fourth row) maps, smoothed on a scale of $\sim 5.3 \mathrm{kpc}$ to reduce noise and then divided by maps smoothed on a larger scale of $\sim 26.4 \mathrm{kpc}$, is shown to highlight the existence of sound waves as enhancements and depressions in the corresponding maps. For the run without substructures (first and second columns), the almost circular shape of the sound waves is clearly seen. It is also evident that as the buoyant rise of the lobes slows below $c_{\mathrm{s}, \mathrm{ICM}}$, the bow shock transitions into a sound wave and detaches itself from the motion of the lobes. On the other hand, sound waves and weak shocks produced by substructure stirring completely disrupt the cocoon in the run with substructures (third and fourth columns), and hence, we do not readily detect the large sound wave in this run. We note that we do not include a description for physical viscosity in our simulations, and so any dissipation of, and hence heating due to, the sound waves is purely numerical.

Finally, as the jet and sound wave propagate through the ICM, they perturb the ICM, which after their passage can continue to oscillate. Omma et al. (2004) classified similar oscillations in their simulations, as g-modes excited within the ICM. In our case, this would be consistent with the generation of vorticity perpendicular to the jet motion discussed in Section 4.2. The gravity waves are confined to the region within the expanding sound wave and have a very 
small amplitude. While a full analysis of their energetics is beyond the scope of this paper, it is expected that they make an insignificant contribution to ICM heating (Reynolds et al. 2015).

\section{DISCUSSION}

\subsection{Numerical Jets}

In Section 3, we compared the impact of various numerical parameters and different energy injection techniques on jet evolution. We compared the evolution of jet length with simple analytical predictions for jet evolution, based on those of Begelman \& Cioffi (1989). We found that the jet behaves as expected based on this simple model, but only when the evolution of the jet cross-section and momentum rate are taken into account (e.g. Norman et al. 1982; Lind et al. 1989; Krause \& Camenzind 2001; Krause 2003). However, to successfully model the propagation and evolution of a jet, one must make careful consideration to the refinement scheme implemented within the simulation. We have shown that an overly aggressive de-refinement scheme can stunt jet evolution, inhibit growth and promote numerically driven mixing.

A number of energy injection schemes are used within the literature. Earlier works (e.g. Cattaneo \& Teyssier 2007) injected momentum and thermal energy, while more recent work (e.g. Gaspari et al. 2011; Prasad et al. 2015; Yang \& Reynolds 2016a,b) implement purely kinetic jets, thus motivating us to test different schemes. While the thermal jets may seem more physically motivated, given that momentum is intrinsically conserved, they can significantly alter the entropy profiles of galaxy clusters and readily destroy cool cores (Cattaneo \& Teyssier 2007). On the other hand, it has been shown in the literature that purely kinetic jets are able to maintain cluster cool cores (Gaspari et al. 2011; Yang et al. 2012). We find that although the majority of the jet kinetic energy is thermalized through shocks within the jet lobe, irrespective of the chosen energy injection technique, there are still differences in jet morphology. While the purely kinetic injection produces longer jets, more akin to an FR-II morphology, the thermal and precessing kinetic jets appear much closer to the FR-I jets seen in galaxy clusters.

The requirement for jets to precess in order to efficiently and isotropically heat the ICM was proposed by Vernaleo \& Reynolds (2006), and has since been implemented in a number of works as a necessary ingredient (e.g. FalcetaGonçalves et al. 2010; Li \& Bryan 2014; Yang \& Reynolds $2016 \mathrm{a}, \mathrm{b})$. The precessing jet we consider is the same as the kinetic jet except that the direction of the jet precesses about the $z$-axis at an angle of $15^{\circ}$ with a period of $\sim 10 \mathrm{Myr}$, similar to the implementation of Yang \& Reynolds (2016a). Morphologically, the precessing jet appears similar to the thermal jet, with a seemingly more isotropic jet lobe distribution. In terms of energetics, a larger fraction of the initial kinetic energy of the precessing jet is converted into thermal energy through shocks due to the extra jet motions. Despite the morphological differences, as discussed in Section 4.3, both the fiducial kinetic and precessing kinetic jets produce rather similar radial ICM profiles, with the precessing jet only being moderately better at preventing cold material reaching the central regions of the halo. However, we note that Meece et al. (2017) have recently compared different precession angles, finding that larger values of $\theta_{\text {prec }}$ result in a larger fraction of jet kinetic energy thermalizing through shocks.

Observations of the locations of relic X-ray lobes suggest that AGN jets may be able to move, while the shape of observed jet emission further suggests precession (e.g. Dunn et al. 2006a; Martí-Vidal et al. 2011; Babul et al. 2013). Although as shown in Section 5.6, lobes can also be displaced by ICM bulk motions. More recent observations of the jet and molecular outflow in NGC 1377 (Aalto et al. 2016) show kinematic behaviour that is also consistent with jet precession, as is also found in the line-of-sight velocities of our precessing jet model. The precession of jets produced by SMBHs is still debated in the literature, with the exact mechanism driving the precession not clearly understood. Nixon \& King (2013) have considered whether or not jets are physically able to precess or even move based upon either the evolution of the orientation of the BH spin or inner accretion disc angular momentum. They suggest that for massive BHs, such as those in AGN, it is very difficult to significantly change the $\mathrm{BH}$ spin direction during a single accretion event and that jet precession time-scales of less than the accretion time-scale would imply that it is the accretion disc driving the jet, opposed to the spin. In this case the jet precession could potentially be driven by self-induced warping of an irradiated accretion disc (Pringle 1996, 1997) or by accretion discs that tear (Nixon et al. 2012a,b) due to the Lense-Thirring effect (Lense \& Thirring 1918). On the other hand, alternative models also suggest that massive $\mathrm{BH}$ binaries could also result in jet precession (Begelman et al. 1980).

\subsection{Jet inflation and velocity structure}

The jet lobe inflation and cocoon development proceed through the interaction of the jet with the ambient ICM. Somewhat akin to AGN-driven winds (Faucher-Giguère \& Quataert 2012; Zubovas \& King 2012), the jets collide with and shock against the ICM. This produces the jet lobes, full of shocked jet material, and the rest of the jet cocoon. Initially, while the cocoon material expands faster than $c_{\mathrm{s}, \mathrm{ICM}}$, the cocoon consists of shocked ICM material. However, once the expansion slows, the shock broadens into a sound wave expanding into the ICM. The general structure of these regions is illustrated in Fig. 1, which shows the four main regions in the vicinity of a jet. The shocked jet material expands thermally, producing the jet lobes, while the cocoon expands perpendicular to the jet direction either as a shock wave or a sound wave, depending on the gas radial velocity. While the propagation of the jet in the $z$-direction drives a bow shock into the ICM, which is dominated by ram pressure, the shocked jet and ICM material is dominated by thermal pressure. This is in line with the theoretical structure of jets outlined by analytical models (e.g. Blandford \& Rees 1974; Scheuer 1974; Begelman \& Cioffi 1989) and simulations (e.g. Norman et al. 1982; Lind et al. 1989; Krause \& Camenzind 2001; Krause 2003; Hardcastle \& Krause 2013).

As discussed in Section 4.2, at later times, ICM material displaced by the inflation of the jet cocoon flows into the centre of the galaxy cluster, mainly through the plane perpendicular to the jet direction. Some of this material then 
appears to be dragged up by the motion of the jet before falling back into the cluster potential well, resulting in a gas circulation towards the base of the jet, similar in fashion to a galactic fountain. We also note that backflows, expected to arise due to steep gradients in entropy and density (e.g. Antonuccio-Delogu \& Silk 2010; Cielo et al. 2014), form within the jet cocoon. However, especially at later times, we find that these are readily disrupted by jet-driven turbulence.

\subsection{Heating mechanisms and the energy budget}

The combined thermal and kinetic energy content of the hotjet-enriched lobe material $\left(f_{\mathrm{J}}>0.01\right)$ makes up $\sim 30$ per cent of the total injected energy and is dominated by the thermal component. We find that significant vorticity, and hence turbulence, is only produced within the jet lobe material (see also Weinberger et al. 2017) and given that most of the energy content of the jet lobe is thermalized through shocks, we suggest that the jet is unable to drive significant turbulence in the ICM. After $45 \mathrm{Myr}$, the remaining $\sim 70$ per cent of energy injected by the jet, which does not reside in the jet-enriched lobe gas, either goes in to less jet-rich lobe gas $\left(0.001<f_{\mathrm{J}}<0.01, \sim 10\right.$ per cent $)$, the kinetic energy of the expanding cocoon or other kinetic motions, the gravitational potential energy of gas lifted out of the potential well of the cluster, heating of the ICM (see below) or is lost to radiative cooling and adiabatic expansion. Considering the kinetic run without radiative cooling (see Fig. 5), $\simeq 64$ per cent of the injected energy goes into the thermal component, with about half of this being in ICM gas with $f_{\mathrm{J}}<0.001$. Given that the jet is unable to drive significant turbulence within the ICM and that we do not find significant mixing of jet lobe and ICM material, we suggest that this ICM heating, at least in the adiabatic case, is primarily due to compression and weak shocks (see also Yang \& Reynolds 2016b). We note that this partitioning of the energy is achieved while the jet is active and will likely change as the system evolves once a jet becomes inactive.

As outlined in the Introduction section, a number of channels have been proposed for converting the kinetic energy of the jet into thermal energy within the ICM to suppress cooling. The most direct interaction between the jet and the ICM is through shocks. While we find that a significant fraction of the kinetic energy of the jet is thermalized within the jet lobes themselves, the expansion of the cocoon is significantly less effective at driving strong shocks into the ICM. Although a continuous bow shock is driven in the jet direction, the perpendicular expansion of the cocoon only drives a shock wave into the ICM during the first few Myr of jet activity. This then transitions into a sound wave propagating into the ICM (see Section 5.6). The lack of strong ICM shocks in our simulations is consistent with the fact that observed AGN-driven shocks in galaxy clusters are often weak (e.g. Fabian et al. 2006; Forman et al. 2007; Croston et al. 2011; Sanders et al. 2016) and are not expected to provide enough energy to be the dominant contribution to the heating of the ICM, although may be able to prevent cooling close to the BH (Nulsen et al. 2007). Indeed, Yang \& Reynolds (2016a) found that while weak shocks can heat the ICM, they cannot overcome radiative cooling and only result in a 'reduced cooling flow', while Li et al. (2016) found that shock heating can provide an order of magnitude greater heating than turbulent heating. It is also interesting to note that, as shown in Section 5.6, once the jet becomes inactive and the buoyantly rising bubbles slow down, the sound wave produced by the jet inflation 'detaches' from the jet lobes and can propagate to large distances through the ICM (see also Guo et al. 2017). Similarly, sound waves have been observed in galaxy clusters (e.g. Fabian et al. 2003, 2005b), but the exact details of how and where the energy carried by these sound waves is dissipated depends upon the form of the physical viscosity of the ICM (e.g. Ruszkowski et al. 2004; Sijacki \& Springel 2006c).

It has been suggested that a significant amount of energy can go into the form of cavity heating (e.g. Churazov et al. 2002; Bîrzan et al. 2004; Nulsen et al. 2007), whereby the potential energy of the material displaced by the expansion of the jet lobes can be converted into kinetic energy and subsequently heat. As highlighted in Section 4.2, the jet lobes inflated in our simulations are able to displace large quantities of ambient ICM gas $\left(\sim 10^{10}-10^{11} \mathrm{M}_{\odot}\right.$ by 45 Myr, depending on energy injection method), some of which likely falls into the potential well of the cluster. Thus the jet action not only stimulates the conversion of ICM gravitationally potential energy into kinetic energy and heat but also provides further fuel for $\mathrm{BH}$ growth.

Finally, mixing of jet material could play a role in communicating the thermalized jet energy within the lobes to the ICM. Similarly to Yang \& Reynolds (2016a), we find that there can be mixing of jet material within the jet lobes, but find negligible amounts of jet material beyond their immediate vicinity while the jet is active. The evolution of the jet lobes once the jet switches off depends upon whether or not substructures have stirred the ICM. In the run without substructures, the jet lobes rise buoyantly through the ICM and are gradually disrupted by fluid instabilities. Additionally, the motions of substructures displace the rising lobes from their original trajectory and further promote mixing. We find that $\sim 450$ Myr after the jet switched off, substructures can promote mixing by a factor of up to $\sim 3-4$. Interestingly, Hillel \& Soker (2016, 2017a) suggest from their simulations that mixing of bubble material with the ICM actually plays a more dominant role than turbulent and shock heating. However, we note that the draping of magnetic field lines over the jet lobes, even in a weak magnetic field, could have an important impact on the evolution and dynamics of jet lobes (e.g. Dursi \& Pfrommer 2008). While recent magnetohydrodynamic simulations have found that mixing is less efficient than in purely hydrodynamic simulations (e.g. Weinberger et al. 2017). On the other hand, it has also been shown that anisotropic thermal conduction can in fact increase mixing and promote isotropization of injected feedback energy (Kannan et al. 2017).

\subsection{Substructures and turbulence in the ICM}

In Section 5, we built upon the idealized simulations presented in Section 3 by including substructures, which were added by hand. We found that the motions of the substructures are able to produce significant vorticity within the ICM and drive turbulence, with the total kinetic energy of gas accounting for $\sim 9-30$ per cent of the total kinetic plus thermal energy within the cluster virial radius. We have shown 
that while jet induced motions are able to drive turbulence and dominate the velocity power spectrum on scales smaller than the jet length, any large-scale turbulence is likely to be driven by substructure motions within the cluster. In fact, the line-of-sight velocities and velocity dispersions measured in our simulations with substructures are consistent with those measured by Hitomi observations of the Perseus cluster (Hitomi Collaboration et al. 2016), suggesting a potentially important role for substructure-driven turbulence in the energy budget of the ICM. However, given that a number of different simulations all find levels of turbulence consistent with the Hitomi results (e.g. Reynolds et al. 2015; Yang \& Reynolds 2016a; Hillel \& Soker 2017b; Lau et al. 2017; Weinberger et al. 2017), but reach at different conclusions regarding what heating mechanisms are dominant, we believe that it is not possible to determine the main contribution to the energy budget from Hitomi observations alone.

Observations suggest that turbulence exists within the ICM (Sanders et al. 2010, 2011; Zhuravleva et al. 2012, 2014; Sanders \& Fabian 2013; Pinto et al. 2015; Hitomi Collaboration et al. 2016; Ogorzalek et al. 2017), perhaps contributing $\lesssim 4-40$ per cent of the pressure support within the ICM (e.g. Sanders \& Fabian 2013; Pinto et al. 2015; Hitomi Collaboration et al. 2016). Such turbulence is likely to be produced by large scale processes within the cluster, such as sloshing, accretion, mergers and substructure motion (Dolag et al. 2005; Vazza et al. 2012; Gu et al. 2013; ZuHone et al. 2013; Iapichino et al. 2017; Vazza et al. 2017). However, it has also been proposed that jets could drive turbulence sufficient to offset cooling within galaxy clusters (Banerjee \& Sharma 2014; Zhuravleva et al. 2014). Such turbulence could be produced when AGN feedback excites $g$-modes in the ICM, which then decay into volume-filling turbulence (Reynolds et al. 2015). Analysis presented by Zhuravleva et al. (2014) for the Perseus and Virgo clusters has shown that the observed levels of turbulence would be able to balance loses due to radiative cooling. However, as discussed in Fabian et al. (2017), the propagation velocity of $g$-modes in the Perseus cluster would likely fall an order of magnitude short of that necessary for the dissipation of AGN-driven turbulence to balance radiative cooling. Indeed, when analysing the simulations presented here we found that while the jet is able to drive turbulence within the jet lobes, it is unable to drive large-scale turbulence within the ICM. The inability to drive significant turbulence within the ICM is consistent with previous simulations (e.g. Reynolds et al. 2015; Yang \& Reynolds 2016a; Weinberger et al. 2017). Therefore, the only scenario in which jet-driven turbulence could provide an isotropic source of heat is if the jet lobes fill the cooling radius of the cluster, as in M87 (Forman et al. 2007). However, even in our purely kinetic jet runs we find that the total kinetic energy content of the lobes accounts for only $\sim 4$ per cent $E_{\text {Inj }}$ and therefore does not dominate the energy budget.

\subsection{Limitations of current simulations}

Given the wide dynamical range and vast number of physical processes that could be important in modelling AGN jets in galaxy clusters, there are by necessity a number of limitations to the simulations presented here. The current simulations have used a fixed $\dot{m}$ accretion rate and hence fixed jet power throughout and thus do not include the back reaction of the ICM on to the subsequent accretion rates and jet production. While our set-up has allowed us to make clean comparisons between different jet injection techniques, in order to make a more meaningful interpretation of how jets regulate heating and cooling within the ICM, we will need to include self-consistent accretion and feedback in future work. On top of this, the jet direction, or the axis about which the jet precesses, is fixed in the simulations presented here, while in reality this is expected to be linked to either the $\mathrm{BH}$ spin or accretion disc angular momentum (e.g. Nixon \& King 2013). We hope to remedy these shortcomings in future work by combining the jet feedback model outlined in this paper with a newly developed $\mathrm{BH}$ accretion scheme, which not only tracks the accretion rate of gas on to the $\mathrm{BH}$ but also models the evolution of both the accretion disc and BH spin (Fiacconi, Sijacki \& Pringle, in preparation).

This work also focuses on purely hydrodynamic jets and does not include the effects of magnetic fields, the importance of which, for example through inhibiting mixing, has been highlighted in recent simulation works (e.g. English et al. 2016; Weinberger et al. 2017). However, we find that it is both instructive and important to understand the hydrodynamic evolution of the jets prior to adding further physics, while also presenting a model that can be readily implemented into hydrodynamic cosmological simulations. A further limitation of the current work is one which plagues many other large-scale simulations of jet evolution, in that the hot gas component of the jet lobe is modelled as a nonrelativistic ideal gas. First, beyond temperatures of $T \sim 10^{10}$ $\mathrm{K}$, any electron population will be relativistic, and secondly, it is not clear what the exact composition of physical jets and jet lobes is, nor what is the relative importance of leptonic and hadronic components (e.g. Dunn et al. 2006b; Bîrzan et al. 2008; Croston et al. 2008; Croston \& Hardcastle 2014; Kang et al. 2014; Kawakatu et al. 2016).

Finally, while we have attempted to include the effects of the motions of substructures by introducing them by hand in an idealized system, we include neither a fully live dark matter distribution nor, perhaps more importantly, a fully cosmological galaxy cluster evolved self-consistently as a function of cosmic time. This will potentially impact the large-scale turbulent velocity field and thus the subsequent evolution and interaction of the jet, jet cocoon and ICM. Our goal is to perform such simulations in upcoming work.

\section{SUMMARY}

In this paper, we have presented a novel approach for the simulation of AGN jets in the moving mesh-code AREPO (Springel 2010). The main results of this paper are as follows:

- With an appropriate refinement scheme, we are able to successfully model the injection of a hydrodynamic jet and the subsequent inflation of jet lobes that are consistent with analytical expectations and previous simulation work. Different energy injection methods can result in jets with greatly differing morphologies; however, the total energy content within jet lobe material is remarkably consistent between methods (assuming energy is explicitly conserved in the injection process). 
- The jets are able to affect the energy budget within the central regions of a galaxy cluster, changing the thermal, kinetic and gravitational potential energy content. The jets are able to generate significant levels of vorticity and drive turbulence within the jet lobes. However, such turbulence is not seen on larger scales (unless substructures are included) and we suggest that jets are unable to drive turbulence within a significant fraction of the ICM (see also Reynolds et al. 2015; Yang \& Reynolds 2016a; Weinberger et al. 2017).

- Substructures within the galaxy cluster are able to stir the ICM and generate turbulent motions. This additional velocity field can interact with and disrupt the cocoons inflated by jets, providing additional pressure support, potentially promoting mixing of jet cocoon material with the ICM, and resulting in less symmetric jets.

- Simulations that include substructure motions and a jet are able to produce line-of-sight velocity and velocity dispersion maps, and X-ray emission contours, consistent with those observed in the Perseus cluster by Hitomi Collaboration et al. (2016). Therefore, we conclude that it is possible to produce the low levels of turbulence that are observed within the Perseus cluster through a combination of stirring of the ICM by substructure motions on large scales and jet feedback on smaller scales.

\section{ACKNOWLEDGMENTS}

We would like the thank the anonymous referee for their constructive report on this manuscript. We would also like to thank Christoph Federrath, Tiago Costa and Noam Soker for helpful comments and suggestions. MAB and DS acknowledge support by the ERC starting grant 638707 'BHs and their host galaxies: co-evolution across cosmic time'. DS further acknowledges support from the STFC. This research used: the DiRAC Darwin Supercomputer hosted by the University of Cambridge High Performance Computing Service (http://www.hpc.cam.ac.uk/), provided by Dell Inc. using Strategic Research Infrastructure Funding from the Higher Education Funding Council for England and funding from the Science and Technology Facilities Council; the COSMA Data Centric system at Durham University, operated by the Institute for Computational Cosmology on behalf of the STFC DiRAC HPC Facility. This equipment was funded by a BIS National E-infrastructure capital grant ST/K00042X/1, STFC capital grant ST/K00087X/1, DiRAC Operations grant ST/K003267/1 and Durham University. The DiRAC Complexity system, operated by the University of Leicester IT Services, which forms part of the STFC DiRAC HPC Facility (www.dirac.ac.uk). This equipment is funded by BIS National E-Infrastructure capital grant ST/K000373/1 and STFC DiRAC Operations grant $\mathrm{ST} / \mathrm{K} 0003259 / 1$. DiRAC is part of the UK National EInfrastructure.

\section{REFERENCES}

Aalto S., et al., 2016, A\&A, 590, A73

Antonuccio-Delogu V., Silk J., 2010, MNRAS, 405, 1303

Babul A., Sharma P., Reynolds C. S., 2013, ApJ, 768, 11

Banerjee N., Sharma P., 2014, MNRAS, 443, 687
Basson J. F., Alexander P., 2003, MNRAS, 339, 353

Bauer A., Springel V., 2012, MNRAS, 423, 2558

Begelman M. C., Cioffi D. F., 1989, ApJ, 345, L21

Begelman M. C., Blandford R. D., Rees M. J., 1980, Nature, 287, 307

Binney J., Tabor G., 1995, MNRAS, 276, 663

Bîrzan L., Rafferty D. A., McNamara B. R., Wise M. W., Nulsen P. E. J., 2004, ApJ, 607, 800

Bîrzan L., McNamara B. R., Nulsen P. E. J., Carilli C. L., Wise M. W., 2008, ApJ, 686, 859

Blandford R. D., Rees M. J., 1974, MNRAS, 169, 395

Blanton E. L., Randall S. W., Clarke T. E., Sarazin C. L., McNamara B. R., Douglass E. M., McDonald M., 2011, ApJ, 737, 99

Boehringer H., Voges W., Fabian A. C., Edge A. C., Neumann D. M., 1993, MNRAS, 264, L25

Bogdanović T., Reynolds C. S., Balbus S. A., Parrish I. J., 2009, ApJ, 704, 211

Böhringer H., Matsushita K., Churazov E., Ikebe Y., Chen Y., 2002, A\&A, 382, 804

Bourne M. A., Zubovas K., Nayakshin S., 2015, MNRAS, 453, 1829

Bower R. G., Benson A. J., Malbon R., Helly J. C., Frenk C. S., Baugh C. M., Cole S., Lacey C. G., 2006, MNRAS, 370, 645

Bregman J. N., Fabian A. C., Miller E. D., Irwin J. A., 2006, ApJ, 642, 746

Burns J. O., 1990, AJ, 99, 14

Cattaneo A., Teyssier R., 2007, MNRAS, 376, 1547

Cavagnolo K. W., Donahue M., Voit G. M., Sun M., 2008, ApJ, 683, L107

Churazov E., Brüggen M., Kaiser C. R., Böhringer H., Forman W., 2001, ApJ, 554, 261

Churazov E., Sunyaev R., Forman W., Böhringer H., 2002, MNRAS, 332, 729

Cielo S., Antonuccio-Delogu V., Macciò A. V., Romeo A. D., Silk J., 2014, MNRAS, 439, 2903

Conroy C., Ostriker J. P., 2008, ApJ, 681, 151

Cooke K. C., O'Dea C. P., Baum S. A., Tremblay G. R., Cox I. G., Gladders M., 2016, ApJ, 833, 224

Crawford C. S., Allen S. W., Ebeling H., Edge A. C., Fabian A. C., 1999, MNRAS, 306, 857

Croston J. H., Hardcastle M. J., 2014, MNRAS, 438, 3310

Croston J. H., Hardcastle M. J., Birkinshaw M., Worrall D. M., Laing R. A., 2008, MNRAS, 386, 1709

Croston J. H., Hardcastle M. J., Mingo B., Evans D. A., Dicken D., Morganti R., Tadhunter C. N., 2011, ApJ, 734, L28

Croton D. J., et al., 2006, MNRAS, 365, 11

Curtis M., Sijacki D., 2015, MNRAS, 454, 3445

Dolag K., Vazza F., Brunetti G., Tormen G., 2005, MNRAS, 364, 753

Donahue M., et al., 2010, ApJ, 715, 881

Dubois Y., Devriendt J., Slyz A., Teyssier R., 2010, MNRAS, 409, 985

Dubois Y., Devriendt J., Teyssier R., Slyz A., 2011, MNRAS, 417, 1853

Dubois Y., Devriendt J., Slyz A., Teyssier R., 2012, MNRAS, 420, 2662

Dunn R. J. H., Fabian A. C., 2006, MNRAS, 373, 959

Dunn R. J. H., Fabian A. C., 2008, MNRAS, 385, 757

Dunn R. J. H., Fabian A. C., Taylor G. B., 2005, MNRAS, 364, 1343

Dunn R. J. H., Fabian A. C., Sanders J. S., 2006a, MNRAS, 366, 758

Dunn R. J. H., Fabian A. C., Celotti A., 2006b, MNRAS, 372, 1741

Dursi L. J., Pfrommer C., 2008, ApJ, 677, 993

Egami E., Rieke G. H., Fadda D., Hines D. C., 2006, ApJ, 652, L21 
English W., Hardcastle M. J., Krause M. G. H., 2016, MNRAS, 461, 2025

Fabian A. C., 1994, ARA\&A, 32, 277

Fabian A. C., 2012, ARA\&A, 50, 455

Fabian A. C., et al., 2000, MNRAS, 318, L65

Fabian A. C., Sanders J. S., Allen S. W., Crawford C. S., Iwasawa K., Johnstone R. M., Schmidt R. W., Taylor G. B., 2003, MNRAS, 344, L43

Fabian A. C., Sanders J. S., Taylor G. B., Allen S. W., 2005a, MNRAS, 360, L20

Fabian A. C., Reynolds C. S., Taylor G. B., Dunn R. J. H., 2005b, MNRAS, 363, 891

Fabian A. C., Sanders J. S., Taylor G. B., Allen S. W., Crawford C. S., Johnstone R. M., Iwasawa K., 2006, MNRAS, 366, 417

Fabian A. C., et al., 2011, MNRAS, 418, 2154

Fabian A. C., Walker S. A., Russell H. R., Pinto C., Sanders J. S., Reynolds C. S., 2017, MNRAS, 464, L1

Falceta-Gonçalves D., Caproni A., Abraham Z., Teixeira D. M., de Gouveia Dal Pino E. M., 2010, ApJ, 713, L74

Faucher-Giguère C.-A., Quataert E., 2012, MNRAS, 425, 605

Federrath C., Klessen R. S., 2012, ApJ, 761, 156

Federrath C., Klessen R. S., Schmidt W., 2009, ApJ, 692, 364

Federrath C., Schrön M., Banerjee R., Klessen R. S., 2014, ApJ, 790,128

Federrath C., et al., 2017, in Crocker R. M., Longmore S. N., Bicknell G. V., eds, IAU Symposium Vol. 322, The MultiMessenger Astrophysics of the Galactic Centre. Cambridge University Press, Cambridge, pp 123-128

Ferrarese L., Merritt D., 2000, ApJ, 539, L9

Fogarty K., Postman M., Larson R., Donahue M., Moustakas J., 2017, ApJ, 846, 103

Forman W., et al., 2007, ApJ, 665, 1057

Fujita Y., Suzuki T. K., Wada K., 2004, ApJ, 600, 650

Gaibler V., Krause M., Camenzind M., 2009, MNRAS, 400, 1785

Gaibler V., Khochfar S., Krause M., Silk J., 2012, MNRAS, 425, 438

Gaspari M., Melioli C., Brighenti F., D'Ercole A., 2011, MNRAS, 411,349

Gaspari M., Ruszkowski M., Sharma P., 2012, ApJ, 746, 94

Grisdale K., Agertz O., Romeo A. B., Renaud F., Read J. I., 2017, MNRAS, 466, 1093

Gu L., et al., 2013, ApJ, 767, 157

Gültekin K., et al., 2009, ApJ, 698, 198

Guo F., Duan X., Yuan Y.-F., 2017, preprint, (arXiv: 1705.10916)

Hardcastle M. J., Krause M. G. H., 2013, MNRAS, 430, 174

Hardcastle M. J., Krause M. G. H., 2014, MNRAS, 443, 1482

Häring N., Rix H.-W., 2004, ApJ, 604, L89

Heinz S., Choi Y.-Y., Reynolds C. S., Begelman M. C., 2002a, ApJ, 569, L79

Heinz S., Choi Y.-Y., Reynolds C. S., Begelman M. C., 2002b, ApJ, 569, L79

Hernquist L., 1990, ApJ, 356, 359

Hillel S., Soker N., 2016, MNRAS, 455, 2139

Hillel S., Soker N., 2017a, preprint, (arXiv:1704.07797)

Hillel S., Soker N., 2017b, MNRAS, 466, L39

Hitomi Collaboration et al., 2016, Nature, 535, 117

Hlavacek-Larrondo J., Fabian A. C., Edge A. C., Ebeling H., Sanders J. S., Hogan M. T., Taylor G. B., 2012, MNRAS, 421,1360

Hudson D. S., Mittal R., Reiprich T. H., Nulsen P. E. J., Andernach H., Sarazin C. L., 2010, A\&A, 513, A37

Iapichino L., Schmidt W., Niemeyer J. C., Merklein J., 2011, MNRAS, 414, 2297

Iapichino L., Federrath C., Klessen R. S., 2017, preprint, (arXiv: 1704.02922)

Ikebe Y., et al., 1997, ApJ, 481, 660

Kang S.-J., Chen L., Wu Q., 2014, ApJS, 215, 5
Kannan R., Vogelsberger M., Pfrommer C., Weinberger R., Springel V., Hernquist L., Puchwein E., Pakmor R., 2017, ApJ, 837, L18

Kawakatu N., Kino M., Takahara F., 2016, MNRAS, 457, 1124

Kitsionas S., et al., 2009, A\&A, 508, 541

Kormendy J., Ho L. C., 2013, ARA\&A, 51, 511

Krause M., 2003, A\&A, 398, 113

Krause M., Camenzind M., 2001, A\&A, 380, 789

Lau E. T., Gaspari M., Nagai D., Coppi P., 2017, preprint, (arXiv: 1705.06280)

Lense J., Thirring H., 1918, Physikalische Zeitschrift, 19

Lewis A. D., Stocke J. T., Buote D. A., 2002, ApJ, 573, L13

Li Y., Bryan G. L., 2014, ApJ, 789, 54

Li Y., Bryan G. L., Ruszkowski M., Voit G. M., O'Shea B. W., Donahue M., 2015, ApJ, 811, 73

Li Y., Ruszkowski M., Bryan G. L., 2016, preprint, (arXiv: 1611.05455)

Lind K. R., Payne D. G., Meier D. L., Blandford R. D., 1989, ApJ, 344, 89

Makishima K., et al., 2001, PASJ, 53, 401

Martí-Vidal I., Marcaide J. M., Alberdi A., Pérez-Torres M. A., Ros E., Guirado J. C., 2011, A\&A, 533, A111

Matsushita K., Belsole E., Finoguenov A., Böhringer H., 2002, A\&A, 386, 77

McConnell N. J., Ma C.-P., 2013, ApJ, 764, 184

McDonald M., et al., 2014, ApJ, 794, 67

McNamara B. R., Nulsen P. E. J., 2007, ARA\&A, 45, 117

McNamara B. R., et al., 2000, ApJ, 534, L135

McNamara B. R., Nulsen P. E. J., Wise M. W., Rafferty D. A., Carilli C., Sarazin C. L., Blanton E. L., 2005, Nature, 433, 45

McNamara B. R., et al., 2006, ApJ, 648, 164

McNamara B. R., et al., 2014, ApJ, 785, 44

Meece G. R., Voit G. M., O'Shea B. W., 2017, ApJ, 841, 133

Mittal R., Whelan J. T., Combes F., 2015, MNRAS, 450, 2564

Mittal R., McDonald M., Whelan J. T., Bruzual G., 2017, MNRAS, 465, 3143

Nayakshin S., Zubovas K., 2012, MNRAS, 427, 372

Nixon C., King A., 2013, ApJ, 765, L7

Nixon C. J., King A. R., Price D. J., 2012a, MNRAS, 422, 2547

Nixon C., King A., Price D., Frank J., 2012b, ApJ, 757, L24

Norman M. L., Winkler K.-H. A., Smarr L., Smith M. D., 1982, A\&A, 113, 285

Nulsen P. E. J., Jones C., Forman W. R., David L. P., McNamara B. R., Rafferty D. A., Bîrzan L., Wise M. W., 2007, in Böhringer H., Pratt G. W., Finoguenov A., Schuecker P., eds, Heating versus Cooling in Galaxies and Clusters of Galaxies. p. 210 (arXiv: astro-ph/0611136), doi:10.1007/978-3-54073484-0.37

O'Dea C. P., et al., 2008, ApJ, 681, 1035

Oegerle W. R., Cowie L., Davidsen A., Hu E., Hutchings J., Murphy E., Sembach K., Woodgate B., 2001, ApJ, 560, 187

Ogorzalek A., et al., 2017, MNRAS, 472, 1659

Omma H., Binney J., Bryan G., Slyz A., 2004, MNRAS, 348, 1105

Ostriker J. P., Choi E., Ciotti L., Novak G. S., Proga D., 2010, ApJ, 722, 642

Peterson J. R., et al., 2001, A\&A, 365, L104

Peterson J. R., Kahn S. M., Paerels F. B. S., Kaastra J. S., Tamura T., Bleeker J. A. M., Ferrigno C., Jernigan J. G., 2003, ApJ, 590, 207

Pfrommer C., 2013, ApJ, 779, 10

Pinto C., et al., 2015, A\&A, 575, A38

Prasad D., Sharma P., Babul A., 2015, ApJ, 811, 108

Pringle J. E., 1996, MNRAS, 281, 357

Pringle J., 1997, MNRAS, 292, 136

Quilis V., Bower R. G., Balogh M. L., 2001, MNRAS, 328, 1091

Rafferty D. A., McNamara B. R., Nulsen P. E. J., Wise M. W., 2006, ApJ, 652, 216

Randall S. W., et al., 2015, ApJ, 805, 112 
Reynolds C. S., Heinz S., Begelman M. C., 2001, ApJ, 549, L179

Reynolds C. S., Balbus S. A., Schekochihin A. A., 2015, ApJ, 815, 41

Russell H. R., et al., 2014, ApJ, 784, 78

Russell H. R., et al., 2017, ApJ, 836, 130

Ruszkowski M., Begelman M. C., 2002, ApJ, 581, 223

Ruszkowski M., Oh S. P., 2010, ApJ, 713, 1332

Ruszkowski M., Oh S. P., 2011, MNRAS, 414, 1493

Ruszkowski M., Brüggen M., Begelman M. C., 2004, ApJ, 611, 158

Sanders J. S., Fabian A. C., 2013, MNRAS, 429, 2727

Sanders J. S., Fabian A. C., Smith R. K., Peterson J. R., 2010, MNRAS, 402, L11

Sanders J. S., Fabian A. C., Smith R. K., 2011, MNRAS, 410, 1797

Sanders J. S., et al., 2016, MNRAS, 457, 82

Scheuer P. A. G., 1974, MNRAS, 166, 513

Sijacki D., Springel V., 2006a, MNRAS, 366, 397

Sijacki D., Springel V., 2006b, MNRAS, 366, 397

Sijacki D., Springel V., 2006c, MNRAS, 371, 1025

Sijacki D., Springel V., di Matteo T., Hernquist L., 2007, MNRAS, 380, 877

Sijacki D., Pfrommer C., Springel V., Enßlin T. A., 2008, MNRAS, 387, 1403

Sijacki D., Vogelsberger M., Kereš D., Springel V., Hernquist L., 2012, MNRAS, 424, 2999

Sijacki D., Vogelsberger M., Genel S., Springel V., Torrey P., Snyder G. F., Nelson D., Hernquist L., 2015, MNRAS, 452, 575

Silk J., 2013, ApJ, 772, 112

Springel V., 2005, MNRAS, 364, 1105

Springel V., 2010, MNRAS, 401, 791

Springel V., Hernquist L., 2003, MNRAS, 339, 289

Sun M., 2009, ApJ, 704, 1586

Tamura T., et al., 2001, A\&A, 365, L87

Valdarnini R., 2011, A\&A, 526, A158

Vazza F., Brunetti G., Kritsuk A., Wagner R., Gheller C., Norman M., 2009, A\&A, 504, 33

Vazza F., Roediger E., Brüggen M., 2012, A\&A, 544, A103

Vazza F., Jones T. W., Brüggen M., Brunetti G., Gheller C., Porter D., Ryu D., 2017, MNRAS, 464, 210

Vernaleo J. C., Reynolds C. S., 2006, ApJ, 645, 83

Vogelsberger M., Genel S., Sijacki D., Torrey P., Springel V., Hernquist L., 2013, MNRAS, 436, 3031

Voigt L. M., Fabian A. C., 2004, MNRAS, 347, 1130

Weinberger R., Ehlert K., Pfrommer C., Pakmor R., Springel V., 2017, preprint, (arXiv:1703.09223)

Yang H.-Y. K., Reynolds C. S., 2016a, ApJ, 818, 181

Yang H.-Y. K., Reynolds C. S., 2016b, ApJ, 829, 90

Yang H.-Y. K., Sutter P. M., Ricker P. M., 2012, MNRAS, 427, 1614

Zakamska N. L., Narayan R., 2003, ApJ, 582, 162

Zhuravleva I., Churazov E., Kravtsov A., Sunyaev R., 2012, MNRAS, 422, 2712

Zhuravleva I., et al., 2014, Nature, 515, 85

ZuHone J. A., Markevitch M., Brunetti G., Giacintucci S., 2013, ApJ, 762, 78

Zubovas K., Bourne M. A., 2017, MNRAS, 468, 4956

Zubovas K., King A., 2012, ApJ, 745, L34

von der Linden A., Best P. N., Kauffmann G., White S. D. M., 2007, MNRAS, 379, 867

\section{APPENDIX A: JET MASS}

In our fiducial runs, we haved chosen a jet cylinder mass of $M_{\text {Jet }}=10^{4} \mathrm{M}_{\odot}$, as a balance between resolution and numerical resources. A large $M_{\text {Jet }}$ leads to a poorly resolved jet,
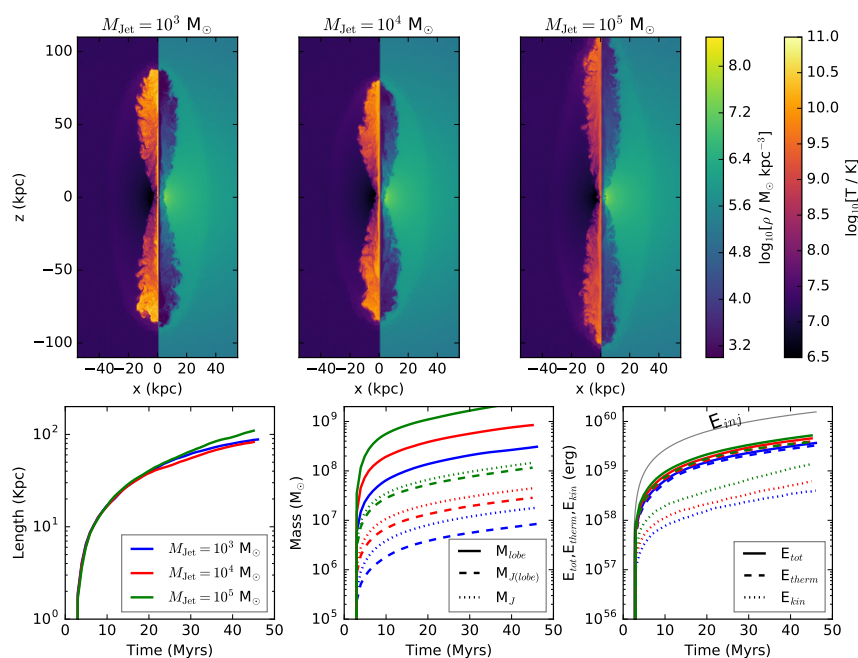

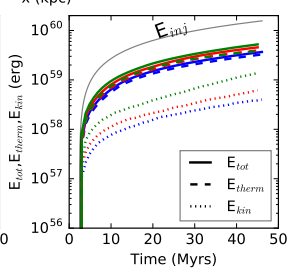

Figure A1. Overview: dependence of jet evolution on jet cylinder mass, which show remarkably similar jet morphologies, although jet masses and kinetic energies correspondingly increase with jet cylinder mass. Top row: density and temperature slices through the $y=0$ plane at $t \simeq 45$ Myr. Bottom row: evolution of the jet length (left-hand panel), different components of jet mass (middle panel) and different components of jet energy content (right-hand panel) for the kinetic runs with $M_{\text {Jet }}=10^{3}$ (left-hand panel and blue curves), $10^{4}$ (middle panel and red curves) and $10^{5} \mathrm{M}_{\odot}$ (right-hand panel and green curves). For comparison, we show the total injected jet energy (equation 24) by the solid black line in the lower right-hand panel.

while a small $M_{\text {Jet }}$ leads to increased run times. To check the impact on jet properties, we have performed additional simulations of kinetic jets with $M_{\text {Jet }}=10^{3}$ and $10^{5} \mathrm{M}_{\odot}$. Density and temperature slices after $t \simeq 45$ Myr for these runs are shown in the top left-hand and right-hand panels of Fig. A1, along with the fiducial $M_{\text {Jet }}=10^{4} \mathrm{M}_{\odot}$ run in the top middle panel. It is clear from these slices that increased jet masses result in higher jet densities and lower temperatures, as approximately the same thermal energy is spread over the larger mass. Additionally, on the bottom row we show the evolution of jet properties, similarly to previous figures.

In all runs, the structure and morphology of the jet appears similar, although the $M_{\mathrm{Jet}}=10^{5} \mathrm{M}_{\odot}$ jet is slightly longer. As expected, increasing $M_{\text {Jet }}$ leads to more massive jets, as shown in the bottom middle panel, with the jet lobe mass, total jet mass and jet mass within the lobe all increasing with $M_{\text {Jet }}$. As shown in the lower right-hand panel, $M_{\text {Jet }}$ also impacts the energy content of the jet, which increases for larger $M_{\mathrm{Jet}}$ and is especially evident for the kinetic energy. If we consider the total energy content within jet lobe material, we find that the $M_{\text {Jet }}=10^{4}$ and $10^{5} \mathrm{M}_{\odot}$ jets retain 24 per cent and 42 per cent more energy in the lobe material compared to the $M_{\text {Jet }}=10^{3} \mathrm{M}_{\odot}$ jet. This again illustrates the difference in mixing between the jet masses.

\section{APPENDIX B: KERNEL FUNCTION}

Both physical and numerical considerations need to be taken into account when choosing a suitable kernel weighting function for mass, momentum and energy injection. In our 

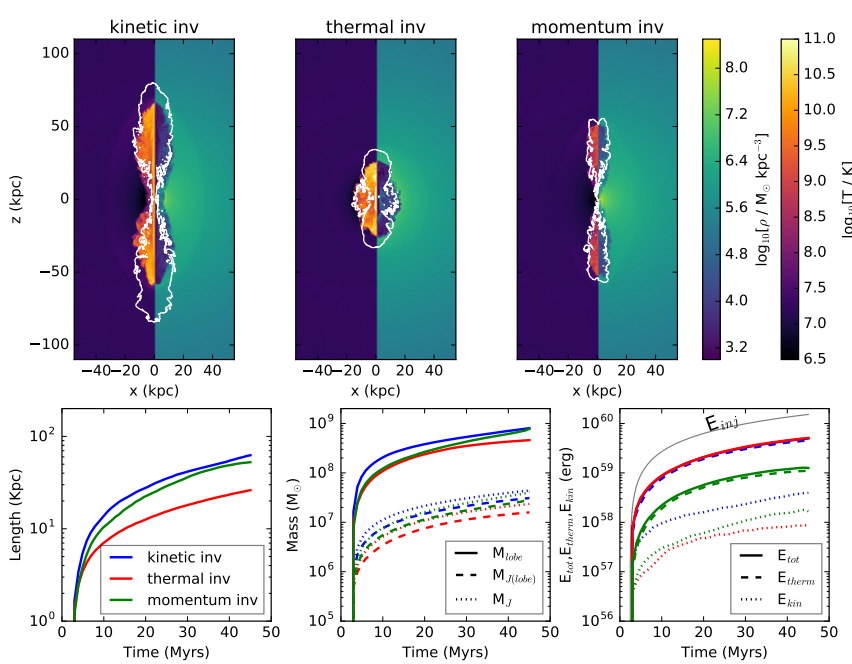

Figure B1. Overview: dependence of jet evolution on the kernel weighting scheme implemented; general jet properties remain similar although inverting the weighting scheme with respect to $z$ can result in broader, shorter jets. Top row: density and temperature slices through the $y=0$ plane at $t \simeq 45$ Myr. Bottom row: evolution of the jet length (left-hand panel), different components of jet mass (middle panel) and different components of jet energy content (right-hand panel) for the kinetic (left-hand panel and blue curves), thermal (middle panel and red curves) and momentum (right-hand panel and green curves) runs, with the modified kernel function. For comparison, we show the total injected jet energy (equation 24) by the solid black line in the lower right-hand panel.

fiducial models, we have chosen a similar kernel weighting scheme as has been used by previous authors (e.g. Omma et al. 2004; Cattaneo \& Teyssier 2007; Yang et al. 2012). Here we consider a modification to the scheme by weighting cells closer to the BH more heavily. This is done by using a kernel of the form

$W_{\mathrm{J}}(r, z) \propto \exp \left(-\frac{r^{2}}{2 r_{\mathrm{Jet}}^{2}}\right)\left(h_{\mathrm{Jet}}-|z|\right)$,

which differs from equation (10) by a factor of $\left(h_{\mathrm{J}}-|z|\right) /|z|$. This results in material close to the $\mathrm{BH}$ receiving a larger kick, reducing the central density and hence resulting in a larger $r_{\text {Jet }}$, when compared to runs with the fiducial kernel function (equation 10). Qualitatively, the impact of this can be seen in the top row of Fig. B1, which shows density and temperature slices similar to those presented in Fig. 4. Additional white contours are included, outlining the shape of the corresponding jets in Fig. 4, for comparison. In general, the structure of the jet is similar, in each case, to those presented previously, with the momentum runs (right-hand panel) being almost identical. However, in the kinetic (lefthand panel) and thermal (middle panel) jet runs, the jets are shorter. The reduced length of the jets can be attributed to the increased jet cylinder radius, $r_{\text {Jet }}$, which results in broader jets and hence a larger ram pressure force acting on the jet along the $z$-axis, as discussed in Section 3.2. The bottom row of Fig. B1 shows jet properties for the kinetic (blue), thermal (red) and momentum (green) runs with the alternative kernel function. In agreement with visual appearance, the overall behaviour of the jets is similar to those presented
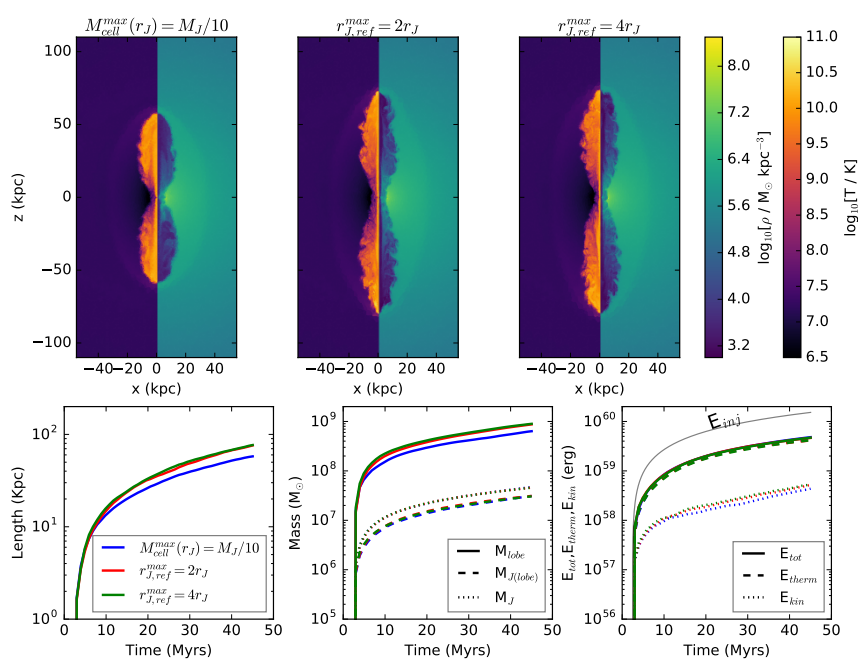

Figure C1. Overview: dependence of jet evolution on jet refinement parameters. Varying the size of the refinement region has a negligible impact on the jet properties; however, increasing the maximum cell size can impact the jet morphology. Top row: density and temperature slices through the $y=0$ plane at $t \simeq 45$ Myr. Bottom row: evolution of the jet length (lefthand panel), different components of jet mass (middle panel) and different components of jet energy content (right-hand panel) when $M_{\text {cell }}^{\max }\left(r_{\text {Jet }}\right)=M_{\text {Jet }} / 10$ (left-hand panel and blue curves), $r_{\text {Jet,ref }}^{\max }=2 r_{\text {Jet }}$ (middle panel and red curves) and $r_{\text {Jet,ref }}^{\max }=4 r_{\text {Jet }}$ (right-hand panel and green curves). For comparison, we show the total injected jet energy (equation 24) by the solid black line in the lower right-hand panel.

in Fig. 4, with the momentum runs being almost identical, while jet lengths in the kinetic and thermal runs are shorter.

\section{APPENDIX C: REFINEMENT PARAMETERS}

The top panels of Fig. C1 show density and temperature slices for jets after $t \simeq 45 \mathrm{Myr}$, while the bottom panel shows the evolution of the jet length (left-hand panel), jet lobe mass components (middle panel) and jet lobe energy components (right-hand panel) when we increase the maximum cell mass at $r=r_{\text {Jet }}$ to $M_{\text {cell }}^{\max }\left(r_{\text {Jet }}\right)=M_{\text {Jet }} / 10$ (top left-hand panel and blue curves; note that the maximum cell mass at $r=0$ is also increased by a factor of 10), decrease the jet refinement region to $r_{\text {Jet,ref }}^{\max }=2 r_{\text {Jet }}$ (top-middle panel and red curves) or increase it to $4 r_{\text {Jet }}$ (top left-hand panel and green curves).

While changing the size of the jet refinement region has little impact on the morphology and physical structure of the jet, allowing larger cell mass results in a shorter jet, likely due to the effective courser jet resolution providing a larger jet working surface radius against the ICM. However, the evolution of the jet lobe mass and, in particular, jet lobe energy content remain remarkably consistent between different parameter choices. We note that due to computational cost, it was not possible to test a significantly reduced value for $M_{\text {cell }}^{\max }\left(r_{\text {Jet }}\right)$ and so the simulations we present provide the balance between resolution and numerical feasibility. 